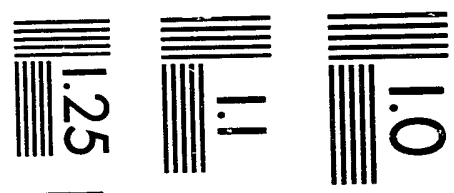

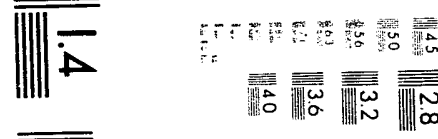

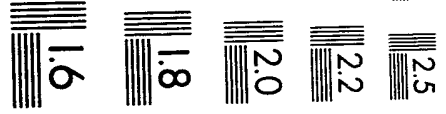



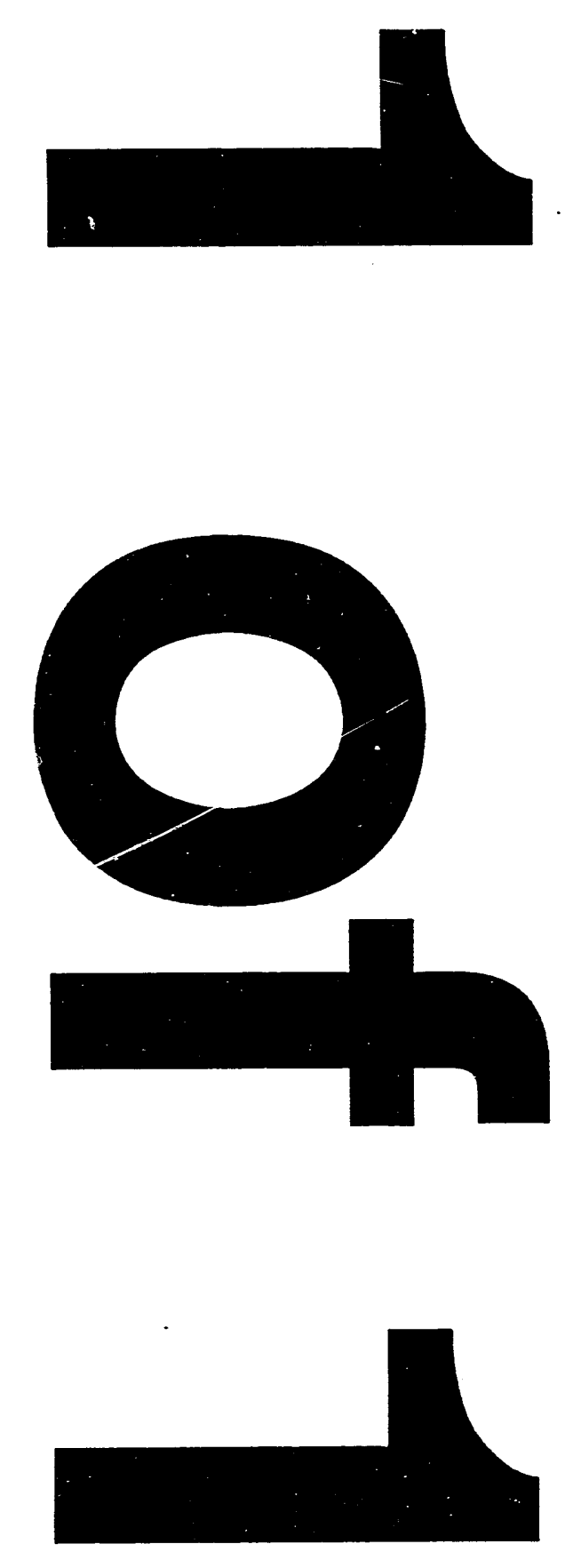


\section{Tube Arrays in Crossflow}

by

S. S. Chen, S. Zhu, and J. A. Jendrzejczyk

Energy Technology Division

June 1993

Work si $\sim$ iorted by

U.S. DEPARTMENT OF ENERGY

Office of Basic Energy Sciences

and

Taiwan Power Company

Taiwan

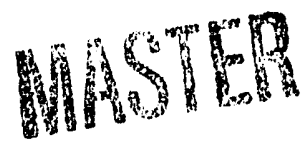

\section{$y$}




\section{Contents}

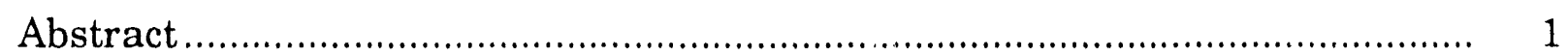

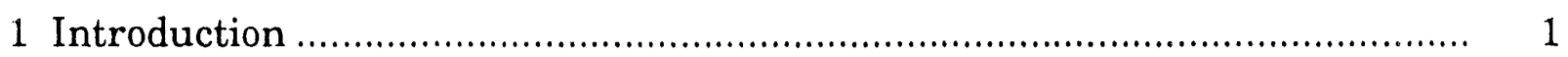

2 Unsteady Flow Theory of Motion-Dependent Fluid Forces ............................ 2

2.1 Quasistatic Flow Theory ..................................................................... 2

2.2 Quasisteady Flow Theory …................................................................

2.3 Unsteady Flow Theory …………........................................................ 4

3 Brief Review of Motion-Dependent Fluid Forces .......................................... 4

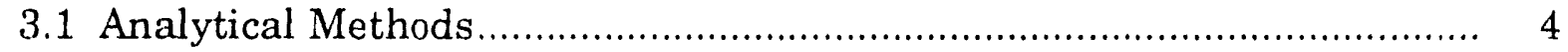

3.2 Numerical Methods .......................................................................... 5

3.3 Experimental Techniques................................................................... 5

4 Experimental Setup................................................................................ 7

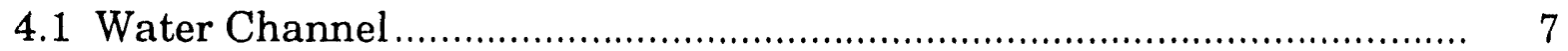

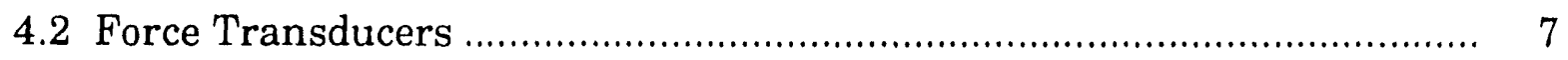

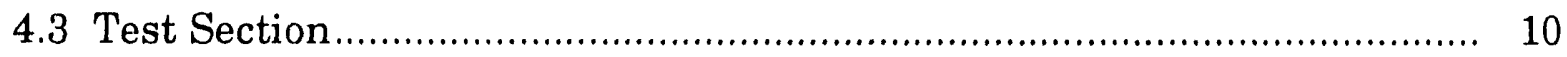

5 Test Procedures and Data Analysis......................................................... 13

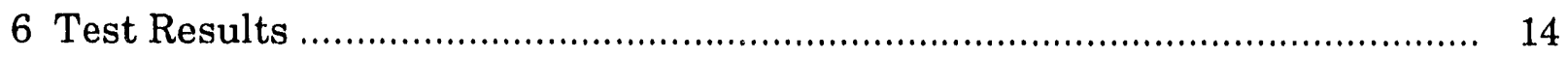

6.1 Added Mass Coefficients ......................................................................... 14

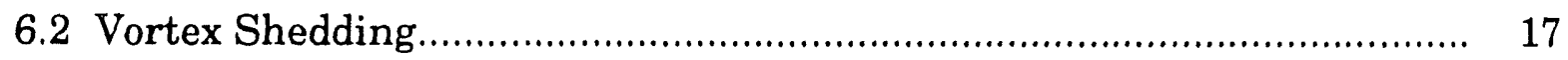

6.3 Motion-Dependent Fluid Forces ........................................................ 18

6.4 Fluid-Force Coefficients...................................................................... 20

6.5 Effects of Oscillation Amplitudes and Excitation Frequencies............... 36

6.6 Symmetry and Antisymmetry of Fluid Forces ...................................... 49

7 Unsteady Flow Theory for Fluidelastic Instability of Tube Arrays................. 51

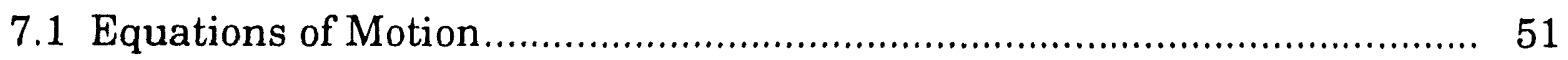

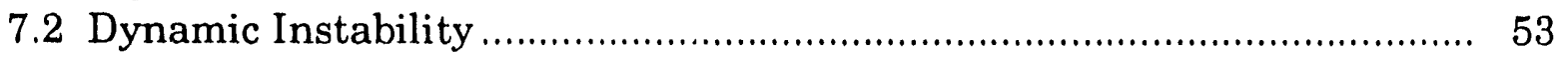

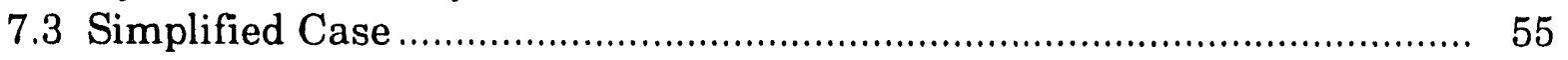

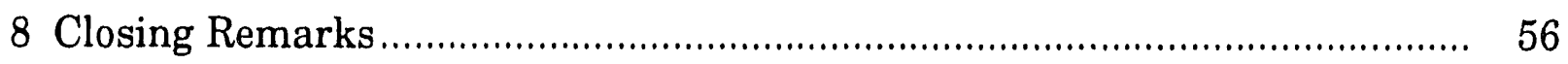


Acknowledgments.

References

\section{Figures}

1 Tube array in crossflow

2 Test channel

3 Water channel.

4 Schematic representation of force transducer

5 Force-to-displacement ratio as a function of excitation frequency for typical cases in which a single tube is oscillating in water.

6 Tube row, square array, and triangular array.

7 Flow diagram of instrumentation for data analysis

8 Schematic representation of first tube array tested, i.e., row of tubes in crossflow.

9 Setup for testing a row of tubes

10 Force-to-displacement ratio as a function of RMS displacement when frequency is 0.5 and $1 \mathrm{~Hz}$

11 Strouhal number for tube row as a function of pitch-to-diameter ratio..

12 Fluid-force components acting on tube row

13 Fluid force $g_{1}$ as a function of tube displacement $v_{1}$ for frequencies of 0.15 and $2 \mathrm{~Hz}$.

14 Magnitude of fluid force and phase angle between fluid force and tube displacement $\mathrm{u}_{1}$ due to oscillation of Tube 1 in the $\mathrm{x}$ and $\mathrm{y}$ directions...

$15 \quad$ Fluid-damping coefficients $\alpha_{11}^{\prime}, \alpha_{21}^{\prime}, \alpha_{31}^{\prime}, \tau_{11}^{\prime}, \tau_{21}^{\prime}$, and $\tau_{31}^{\prime}$, force transducer Type A 
16 Fluid-damping coefficients $\beta_{11}^{\prime}, \beta_{21}^{\prime}, \beta_{31}^{\prime}, \sigma_{11}^{\prime}, \sigma_{21}^{\prime}$, and $\sigma_{31}^{\prime}$ force transducer Type A

$17 \quad$ Fluid-stiffness coefficients $\alpha_{11}^{\prime \prime}, \alpha_{21}^{\prime \prime}, \alpha_{31}^{\prime \prime}, \tau_{11}^{\prime \prime}, \tau_{21}^{\prime \prime}$, and $\tau_{31}^{\prime \prime}$, force transducer Type A

$18 \quad$ Fluid-stiffness coefficients $\beta_{11}^{\prime \prime}, \beta_{21}^{\prime \prime}, \beta_{31}^{\prime \prime}, \sigma_{11}^{\prime \prime}, \sigma_{21}^{\prime \prime}$, and $\sigma_{31}^{\prime \prime}$, force transducer Type A

19 Fluid-damping coefficients $\alpha_{11}^{\prime}, \alpha_{21}^{\prime}, \alpha_{31}^{\prime}, \tau_{11}^{\prime}, \tau_{21}^{\prime}$, and $\tau_{31}^{\prime}$, force transducer Type $B$, reduced flow velocity from 2 to 160

20 Fluid-damping coefficients $\beta_{11}^{\prime}, \beta_{21}^{\prime}, \beta_{31}^{\prime}, \sigma_{11}^{\prime}, \sigma_{21}^{\prime}$, and $\sigma_{31}^{\prime}$, force transduce ' - ype $B$, reduced flow velocity from 2 to 40

$21 \quad$ Fluid-stiffness coefficients $\alpha_{11}^{\prime \prime}, \alpha_{21}^{\prime \prime}, \alpha_{31}^{\prime \prime}, \tau_{11}^{\prime \prime}, \tau_{21}^{\prime \prime}$, and $\tau_{31}^{\prime \prime}$, force transducer Type $B$, reduced flow velocity from 2 to 160

22 Fluid-stiffness coefficients $\beta_{11}^{\prime \prime}, \beta_{21}^{\prime \prime}, \beta_{31}^{\prime \prime}, \sigma_{11}^{\prime \prime}, \sigma_{21}^{\prime \prime}$, and $\sigma_{31}^{\prime \prime}$, force transducer Type $B$, reduced flow velocity from 2 to 40

$23 \quad$ Fluid-damping coefficients $\alpha_{11}^{\prime}, \alpha_{21}^{\prime}, \alpha_{31}^{\prime}, \tau_{11}^{\prime}, \tau_{21}^{\prime}$, and $\tau_{31}^{\prime}$ force transducer Type $B$, reduced flow velocity from 2 to 200

24 Fluid-damping coefficients $\beta_{11}^{\prime}, \beta_{21}^{\prime}, \beta_{31}^{\prime}, \sigma_{11}^{\prime}, \sigma_{21}^{\prime}$, and $\sigma_{31}^{\prime}$, force transducer Type $B$, reduced flow velocity from 2 to 200

$25 \quad$ Fluid-stiffness coefficients $\alpha_{11}^{\prime \prime}, \alpha_{21}^{\prime \prime}, \alpha_{31}^{\prime \prime}, \tau_{11}^{\prime \prime}, \tau_{21}^{\prime \prime}$, and $\tau_{31}^{\prime \prime}$ force transducer Type B, reduced flow velocity from 2 to 200

26 Fluid-stiffness coefficients $\beta_{11}^{\prime \prime}, \beta_{21}^{\prime \prime}, \beta_{31}^{\prime \prime}, \sigma_{11}^{\prime \prime}, \sigma_{21}^{\prime \prime}$, and $\sigma_{31}^{\prime \prime}$ force transducer Type $B$, reduced flow velocity from 2 to 200

$27 \quad$ Fluid-damping coefficients $\alpha_{11}^{\prime}, \alpha_{21}^{\prime}, \alpha_{31}^{\prime}, \tau_{11}^{\prime}, \tau_{21}^{\prime}$, and $\tau_{31}^{\prime}$ as a function of RMS tube displacement oscillating at $0.12 \mathrm{~Hz}$, force transducer Type A

28 Fluid-stiffness coefficients $\alpha_{11}^{\prime \prime}, \alpha_{21}^{\prime \prime}, \alpha_{31}^{\prime \prime}, \tau_{11}^{\prime \prime}, \tau_{21}^{\prime \prime}$, and $\tau_{31}^{\prime \prime}$ as a function of RMS tube displacement oscillating at $0.12 \mathrm{~Hz}$, force transducer Type A 
$29 \quad$ Fluid-damping coefficients $\alpha_{11}^{\prime}, \alpha_{21}^{\prime}, \alpha_{31}^{\prime}, \tau_{11}^{\prime}, \tau_{21}^{\prime}$, and $\tau_{31}^{\prime}$ as a function of RMS tube displacement oscillating at $1.2 \mathrm{~Hz}$, force transducer Type A

$30 \quad$ Fluid-stiffness coefficients $\alpha_{11}^{\prime \prime}, \alpha_{21}^{\prime \prime}, \alpha_{31}^{\prime \prime}, \tau_{11}^{\prime \prime}, \tau_{21}^{\prime \prime}$, and $\tau_{31}^{\prime \prime}$ as a function of RMS tube displacement oscillating at $1.2 \mathrm{~Hz}$, force transducer Type A

$31 \quad$ Fluid-damping coefficients $\alpha_{11}^{\prime}, \alpha_{21}^{\prime}, \alpha_{31}^{\prime}, \tau_{11}^{\prime}, \tau_{21}^{\prime}$, and $\tau_{31}^{\prime}$ as a function of RMS tube displacement oscillating at $0.06 \mathrm{~Hz}$, force transducer Type B

32 Fluid-damping coefficients $\beta_{11}^{\prime}, \beta_{21}^{\prime}, \beta_{31}^{\prime}, \sigma_{11}^{\prime}, \sigma_{21}^{\prime}$, and $\sigma_{31}^{\prime}$ as a function of RMS tube displacement oscillating at $0.06 \mathrm{~Hz}$, force transducer Type B

$33 \quad$ Fluid-stiffness coefficients $\alpha_{11}^{\prime \prime}, \alpha_{21}^{\prime \prime}, \alpha_{31}^{\prime \prime}, \tau_{11}^{\prime \prime}, \tau_{21}^{\prime \prime}$, and $\tau_{31}^{\prime \prime}$ as a function of RMS tube displacement oscillating at $0.06 \mathrm{~Hz}$, force transducer Type B

$34 \quad$ Fluid-stiffness coefficients $\beta_{11}^{\prime \prime}, \beta_{21}^{\prime \prime}, \beta_{31}^{\prime \prime}, \sigma_{11}^{\prime \prime}, \sigma_{21}^{\prime \prime}$, and $\sigma_{31}^{\prime \prime}$ as a function of RMS tube displacement oscillating at $0.06 \mathrm{~Hz}$, force transducer Type B

$35 \quad$ Fluid-damping coefficients $\alpha_{11}^{\prime}, \alpha_{21}^{\prime}, \alpha_{31}^{\prime}, \tau_{11}^{\prime}, \tau_{21}^{\prime}$, and $\tau_{31}^{\prime}$ as a function of RMS tube displacement nscillating at $0.12 \mathrm{~Hz}$, force transducer Type B

36 Fluid-damping coefficients $\beta_{11}^{\prime}, \beta_{21}^{\prime}, \beta_{31}^{\prime}, \sigma_{11}^{\prime}, \sigma_{21}^{\prime}$, and $\sigma_{31}^{\prime}$ as a function of RMS tube displacement oscillating at $0.12 \mathrm{~Hz}$, force transducer Type B

$37 \quad$ Fluid-stiffness coefficients $\alpha_{11}^{\prime \prime}, \alpha_{21}^{\prime \prime}, \alpha_{31}^{\prime \prime}, \tau_{11}^{\prime \prime}, \tau_{21}^{\prime \prime}$, and $\tau_{31}^{\prime \prime}$ as a function of RMS tube displacement oscillating at $0.12 \mathrm{~Hz}$, force transducer Type $B$

$38 \quad$ Fluid-stiffness coefficients $\beta_{11}^{\prime \prime}, \beta_{21}^{\prime \prime}, \beta_{31}^{\prime \prime}, \sigma_{11}^{\prime \prime}, \sigma_{21}^{\prime \prime}$, and $\sigma_{31}^{\prime \prime}$ as a function of RMS tube displacement oscillating at $0.12 \mathrm{~Hz}$, force transducer Type B 


\section{Tables}

1 Calibration constants for three circular-cross-section and three hexagonal-cross-section tubes...................................................... 10

2 Experimental and theoretical values of added mass coefficients with excitation at $0.5 \mathrm{~Hz}$ 


\title{
Motion-Dependent Fluid Forces Acting on Tube Arrays in Crossflow
}

\author{
by
}

S. S. Chen, S. Zhu, and J. A. Jendrzejczyk

\begin{abstract}
Motion-dependent fluid forces acting on a tube array were measured as a function of excitation frequency, excitation amplitude, and flow velocity. Fluiddamping and fluid-stiffness coefficients were obtained from measured motiondependent fluid forces as a function of reduced flow velocity and excitation amplitude. The water channel and test setup provide a sound facility for obtaining key coefficients for fluidelastic instability of tube arrays in crossflow. Once the motion-dependent fluid-force coefficients have been measured, a reliable design guideline, based on the unsteady flow theory, can be developed for fluidelastic instability of tube arrays in crossflow.
\end{abstract}

\section{Introduction}

The components of many heat exchangers and steam generators comprise a group of tubes submerged in crossflow. Fluid flow is a source of energy that can induce vibration and instability. In general, the excitation forces can be divided into two groups. When a tube array is rigid, it disturbs the flow field and the fluid forces acting on the tubes (called fluid excitation forces) are the result of the fluid flow. For example, steady and fluctuating drag and lift forces are typical fluid excitation forces. When the tubes in an array oscillate in the flow, the oscillating motion will disturb the flow field and the fluid forces acting on the tubes will depend on the motion of the tubes. All the fluid-force components that are a function of tube motion are called motion-dependent fluid forces. Typical examples are fluid added mass, fluid damping, and fluid stiffness.

Fluid excitation forces will excite tube vibration and cause forced vibration and resonance. In general, they do not change tube characteristics. On the other hand, motion-dependent fluid forces can change the characteristics of coupled tube/fluid systems and may induce instability. Mathematically, it can be stated that fluid excitation forces appear on the right-hand side of the differential equations that describe the coupled fluid/tube system, whereas motion-dependent fluid forces appear on the left-hand side of the equation. The main objective of this study is to present the motion-dependent fluid forces that act on a tube array. 


\section{Unsteady Flow Theory of Motion-Dependent Fluid Forces}

Consider a group of $\mathrm{n}$ tubes vibrating in a flow as shown in Fig. 1. The axes of the tubes are parallel to one another and perpendicular to the $x-y$ plane. The radius $R$ of each tube is the same, and the fluid is flowing with a gap flow velocity $U$. The displacement components of tube $j$ in the $x$ and $y$ directions are $u_{j}$ and $v_{j}$, respectively. The motion-dependent fluid-force components acting on tube $\mathrm{j}$ in the $\mathrm{x}$ and $\mathrm{y}$ directions are, respectively, $\mathrm{f}_{\mathrm{j}}$ and $\mathrm{gj}$, and are given by Chen (1987b) as

$$
\begin{aligned}
f_{j}= & -\rho \pi R^{2} \sum_{k=1}^{n}\left(\alpha_{j k} \frac{\partial^{2} u_{k}}{\partial t^{2}}+\sigma_{j k} \frac{\partial^{2} v_{k}}{\partial t^{2}}\right)+\frac{\rho U^{2}}{\omega} \sum_{j=1}^{n}\left(\alpha_{j k}^{\prime} \frac{\partial u_{j}}{\partial t}+\sigma_{j k}^{\prime} \frac{\partial v_{k}}{\partial t}\right) \\
& +\rho U^{2} \sum_{j=1}^{n}\left(\alpha_{j k}^{\prime \prime} u_{k}+\sigma_{j k}^{\prime \prime} v_{k}\right)
\end{aligned}
$$

and

$$
\begin{aligned}
g_{j}= & -\rho \pi R^{2} \sum_{k=1}^{n}\left(\tau_{j k} \frac{\partial^{2} u_{k}}{\partial t^{2}}+\beta_{j k} \frac{\partial^{2} v_{k}}{\partial t^{2}}\right)+\frac{\rho U^{2}}{\omega} \sum_{k=1}^{n}\left(\tau_{j k}^{\prime} \frac{\partial u_{j}}{\partial t}+\beta_{j k}^{\prime} \frac{\partial v_{k}}{\partial t}\right) \\
& +\rho U^{2} \sum_{j=1}^{n}\left(\tau_{j k}^{\prime \prime} u_{k}+\beta_{j k}^{\prime \prime} v_{k}\right),
\end{aligned}
$$

where $\rho$ is fluid density; $t$ is time; $\omega$ is circular frequency of tube oscillations; $\alpha_{j k}$, $\beta_{j k}, \sigma_{j k}$, and $\tau_{j k}$ are added mass coefficients; $\alpha_{j k}^{\prime}, \beta_{j k}^{\prime}, \sigma_{j k}^{\prime}$, and $\tau_{j k}^{\prime}$ are fluiddamping coefficients; and $\alpha_{j k}^{\prime \prime}, \beta_{j k}^{\prime \prime}, \sigma_{j k}^{\prime \prime}$, and $\tau_{j k}^{\prime \prime}$ are fluid-stiffness coefficients.

Motion-dependent fluid forces depend on deviation from a reference state of steady flow, which can be grouped according to three different theories: quasistatic flow, quasisteady flow, and unsteady flow.

\subsection{Quasistatic Flow Theory}

At any instant in time, the fluid-dynamic characteristics of tubes oscillating in a flow are equal to the characteristics of the same stationary tubes whose configuration is identical to the actual instantaneous configuration. The fluid forces depend on the deviation from a reference state of steady flow, i.e., the fluid forces depend only on $u_{j}$ and $v_{j}$, not on $\dot{u}_{j}, \dot{v}_{j}$, $\ddot{u}_{j}$, and $\ddot{v}_{j}$ (the dot denotes differentiation with respect to t), so that 

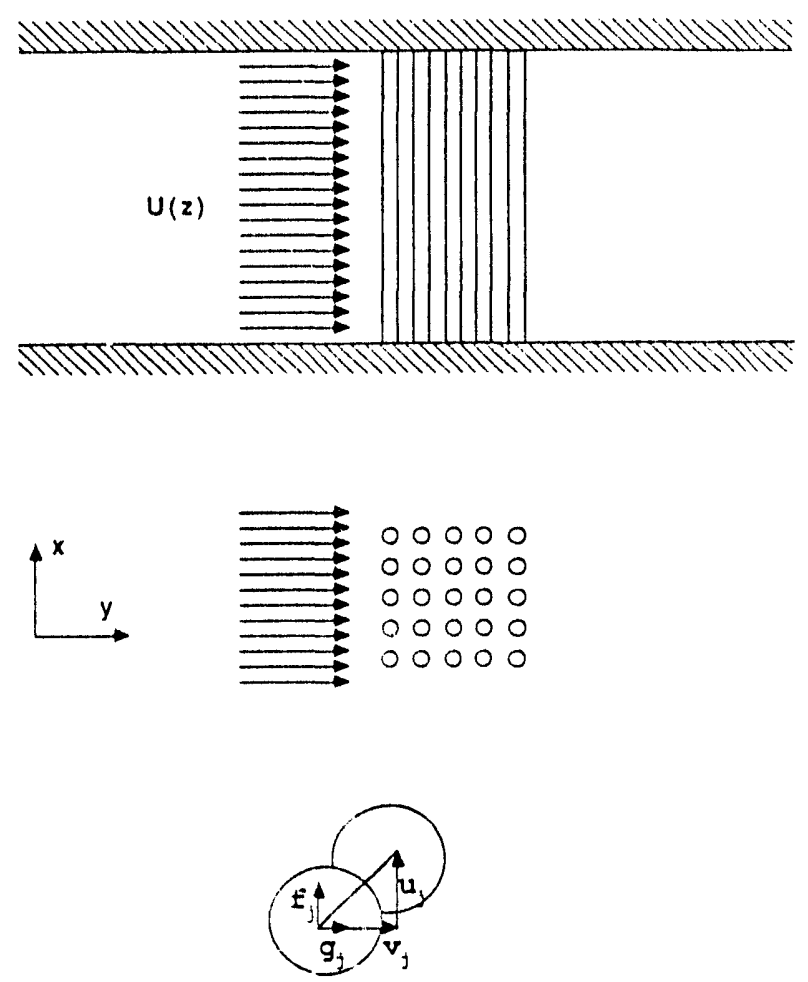

Fig. 1. Tube array in crossflow

$$
f_{j}=\rho U^{2} \sum_{j=1}^{n}\left(\alpha_{j k}^{\prime \prime} u_{k}+\sigma_{j k}^{\prime \prime} v_{k}\right)
$$

and

$$
g_{j}=\rho U^{2} \sum_{j=1}^{n}\left(\tau_{j k}^{\prime \prime} u_{k}+\beta_{j k}^{\prime \prime} v_{k}\right) .
$$

In this case, the fluid forces are determined uniquely by the tube configuration.

\subsection{Quasisteady Flow Theory}

At any instant in time, the fluid-dynamic characteristics of tubes moving in flow are equal to the characteristics of the same tubes moving with constant velocities equal to the actual instantaneous values. The fluid forces depend on tube configuration and are proportional to tube motion. This is reflected by the changes of amplitude and phase of the fluid force with respect to tube motion. In this case, 
the fluid-force components are given by Eqs. 1 and 2. The fluid-stiffness coefficient matrices are constant, i.e., $\alpha_{j k}^{\prime \prime}, \beta_{j k}^{\prime \prime}, \sigma_{j k}^{\prime \prime}$, and $\tau_{j k}^{\prime \prime}$, and are independent of the reduced flow velocity. The fluid-damping coefficient matrices are functions of the reduced flow velocity.

\subsection{Unsteady Flow Theory}

In general, the fluid-force components are nonlinear functions of $u_{j}, v_{j}, \dot{u}_{j}, \dot{v}_{j}, \ddot{u}_{j}$, and $\ddot{v}_{j}$. The general expressions for the fluid-force components are given in Eqs. 1 and 2; however, the fluid-force matrices and fluid-coefficient matrices are functions of $U, u_{j}, v_{j}, \dot{u}_{j}, \dot{v}_{j}, \ddot{u}_{j}$, and $\ddot{v}_{j}$.

\section{Brief Review of Motion-Dependent Fluid Forces}

The crux of stability analysis of tube arrays in crossflow is the information on motion-dependent fluid-force coefficients, in particular, fluid-damping coefficients and fluid-stiffness coefficients. Several approaches can be used to obtain these coefficients, including analytical and numerical methods and experimental techniques.

\subsection{Analytical Methods}

A series of attempts has been made to analyze the fluid forces acting on tube arrays oscillating in crossflow. Theoretically, this analysis should be based on the Navier-Stokes or Reynolds equation. Various approximate methods are used to analyze this problem because of the difficulty in solving the problem exactly.

Potential-flow theory. An investigation of fluid forces based on the potentialflow theory was performed by Chen (1976). The main conclusion of the investigation was that the potential-flow theory is applicable to added mass coefficients but, in general, it is not applicable to fluid-damping and fluid-stiffness coefficients. Additional studies were made by Paidoussis, Price, and Mavriplis (1984) and Van der Hoogt and Van Campen (1984).

Quasistauc flow theory. Typical examples are those by Connors (1970) and Blevins (1977); however, the fluid-stiffness forces are measured experimentally. At this time, no analytical solution is available for the quasistatic flow theory.

Quasisteady flow theory. Several forms of fluid forces based on the quasisteady-flow theory were presented by Price and Paidoussis (1983 and 1984) 
and Paidoussis, Price, and Mavriplis (1984). Some heuristic parameters are also included to improve the quasisteady flow theory.

Unsteady flow theory. Lever and Weaver (1982, 1984) formulated a onedimensional model based on mass and momentum conservation equations, and conducted a series of experiments to verify the basic assumption. Additional studies, by Yetisir and Weaver $(1988,1992)$, were conducted to improve the theory. Another approach, which uses a vorticity formulation for two-dimensional fluid motion, was developed by Marn and Catton (1991).

\subsection{Numerical Methods}

Effective methods for working with moving boundary problems with small-tomedium deformations are based on various algorithms, including Eulerian, Lagrangian, and Eulerian-Lagrangian. For example, the wake interference behind two flat plates normal to the flow was numerically calculated by a finite-element method for two Reynolds numbers, 80 and 160 (Behr, Tezduyar, and Higuchi 1991). The flow patterns were found to vary with Reynolds number and gap size. At this time, although several algorithms are available to work with the motion-dependent fluid forces, no systematic study has been conducted for tube arrays.

\subsection{Experimental Techniques}

The assumption of quasisteady flow was considered by Price, Paidoussis, and Sychtera (1988) for two tubes, one behind the other. It was found that the discrepancy between the quasisteady and unsteady fluid-force coefficients depends not only on reduced flow velocity but also on the position of the leeward tube relative to the windward tube. When reduced flow velocity is large, the quasisteady flow theory is applicable; however, greater caution is required when the quasisteady flow assumption is applied to small reduced flow velocity.

One method to obtain fluid forces is to base the calculation of force coefficients on measured structural responses, such as accelerations and displacements. In some cases, because direct measurement of fluid forces is difficult, e.g., measurements of high buildings or heat exchanger tubes, some attempts have been made to use the inverse method (Xie 1988, and Holzdeppe and Ory 1988). The technique is useful when detailed dynamies of the system are well known, such as in a simple bridge section (Xie 1988). Granger (1990) also used an inverse method to obtain fluid forces. He studied a flexible tube in an otherwise rigid tube bundle that was subjected to water crossflow. Some fluid forces compare reasonably well with the data of Tanaka and Takahara (1981) and Tanaka, Takahara, and Ohta 
(1982). For a group of flexible tubes, it will be difficult to obtain all fluid-force coefficients.

Several experiments focused on measuring motion-dependent fluid forces directly. Teh and Goyder (1988) measured fluid forces acting on a tube that was excited to oscillation. These fluid forces were related to the oscillating tube only; therefore, they can be used for fluid-damping-controlled instability only. Hara (1987) measured unsteady fluid forces acting on a tube row and studied the detailed flow field. Funakawa et al. (1990) performed an experimental study of unsteady fluid forces acting on tube arrays with a pitch ratio of 1.41 in two-phase flow. However, these authors only measured a single component of the fluid forces for a specific motion. The results provide some insights into the instability of tube arrays in two-phase flow but cannot be used for practical prediction of instability. The most extensive measurements of motion-dependent fluid forces were by Tanaka (1980); Tanaka and Takahara (1981); and Tanaka, Takahara, and Ohta (1982), who measured motion-dependent fluid forces for tube rows and square arrays with pitch ratios of 1.33 and 1.42 . This technique was also used by Jendrzejczyk and Chen (1987).

In this study, we used the unsteady flow theory. Fluid-force coefficients can be determined by measuring the fluid forces acting on the tubes that are due to oscillations of a particular tube. For example, if tube $\mathrm{k}$ is excited in the $\mathrm{y}$ direction, its displacement in the $y$ direction is given by

$$
\mathrm{v}_{\mathrm{k}}=\mathrm{v} \cos \omega \mathrm{t} .
$$

The fluid force acting on tube $\mathrm{j}$ in the $\mathrm{x}$ direction can be written

$$
f_{j}=\frac{1}{2} \rho U^{2} c_{j k} \cos \left(\omega t+\phi_{j k}\right) v,
$$

where $c_{j k}$ is the fluid-force amplitude and $\phi_{j k}$ is the phase angle by which the fluid force acting on tube $\mathrm{j}$ leads the displacement of tube $\mathrm{k}$.

With Eqs. 1 and 5, we can also write the fluid-force component as

$$
f_{j}=\left(\rho \pi R^{2} \omega^{2} \sigma_{j k}+\rho U^{2} \sigma_{j k}^{\prime \prime}\right) v \cos \omega t-\rho U^{2} \sigma_{j k}^{\prime} v \sin \omega t
$$

Combining Eqs. 6 and 7 yields

$$
\sigma_{\mathrm{jk}}^{\prime \prime}=\frac{1}{2} c_{\mathrm{jk}} \cos \phi_{\mathrm{jk}}-\frac{\pi^{3}}{\mathrm{U}_{\mathrm{r}}^{2}} \sigma_{\mathrm{jk}}
$$


and

$$
\sigma_{j k}^{\prime}=\frac{1}{2} c_{j k} \sin \phi_{j k}
$$

where $U_{r}$ is the reduced flow velocity $\left(U_{r}=\pi U / \omega R\right)$.

The added mass coefficient $\sigma_{\mathrm{jk}}$ in Eq. 8 can be calculated by applying the potential-flow theory (Chen 1975, 1987a). Then $\sigma_{j k}^{\prime}$ and $\sigma_{j k}^{\prime \prime}$ can be calculated from Eqs. 8 and 9, when the force amplitude $c_{j k}$ and phase angle $\phi_{j k}$ are measured. Other fluid-force coefficients can be obtained in the same manner.

Fluid-force coefficients depend on tube arrangement, tube pitch, oscillation amplitude, oscillation frequency, and flow velocity. For a given tube array, fluidforce coefficients are a function of oscillation amplitude (d/D) and reduced flow velocity $\left(U_{r}\right)$, where $d$ is vibration amplitude and $D$ is tube diameter. For smallamplitude oscillations, fluid-force coefficients can be considered a function of reduced flow velocity only.

\section{Experimental Setup}

\subsection{Water Channel}

The test channel is shown in Figs. 2 and 3. Water is pumped into an input tank. The flow passes through a series of screens and honeycombs and then into a rectangular flow channel. The water level is controlled by standpipes in the output tank and the flow is controlled by the running speed of the pump motor.

Flow velocity is measured with a current flow meter. The rate of propeller rotation is directly proportional to stream velocity and therefore the sensor output signal is not effected by other factors, such as water conductivity, temperature, and suspended particulates.

\subsection{Force Transducers}

A schematic representation of a force transducer is shown in Fig. 4a. The relatively rigid main bodies of the tubes are constructed from stainless steel tubing with a $2.54-\mathrm{cm}(1-\mathrm{in}$.$) OD, a 0.071-\mathrm{cm}(0.028$-in.) wall thickness, and a $38.1-\mathrm{cm}$ (15-in.) length. Thin brass end caps are soldered to both ends of each tube and a smaller, relatively flexible tube, with a $0.635-\mathrm{cm}(0.25$-in.) OD, a $0.089-\mathrm{cm}(0.035$ in.) wall thickness, and a 12.07- $\mathrm{cm}(4.75$-in.) length, is fastened to the upper end cap of each tube (Fig. 4b). Two sets of strain gauges are placed on the outer surface of 


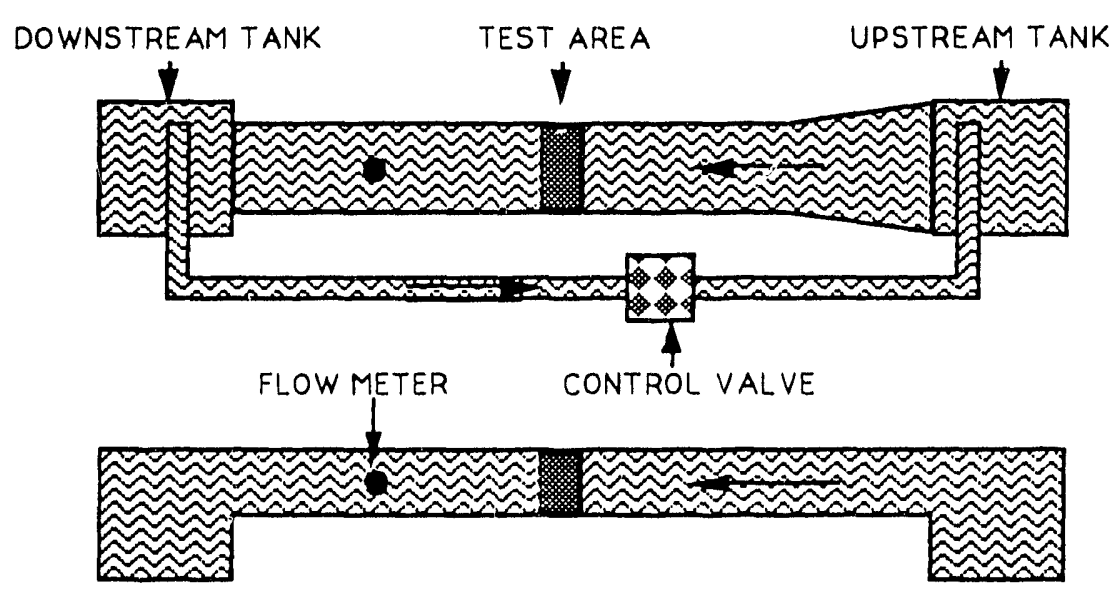

Fig. 2. Test channel

the smaller tube where the outer surface of the tube has been machined to a smaller diameter or a hexagonal section (Fig. 4c). When the cross section is circular the transducer is denoted Type $A$; when it is hexagonal, the transducer is denoted Type B. The two sets of strain gauges measure the force components in the two perpendicular directions with a sensitivity of $\approx 1 \mathrm{~V}$ for $0.05 \mathrm{~N}(0.01 \mathrm{lb})$ of force acting on the middle of the active tubes.

The force transducers are calibrated by two methods, static and dynamic. In the static method in air, the active tube is held fixed at the supported end and a given force is applied to the middle of the active length. In the dynamic method in air and water, the tube is excited at a given frequency and amplitude in air or water. Then, the inertia forces due to the sinusoidal oscillations are used to determine the calibration constant.

All calibration constants used in the measurement are determined in water. For a single tube vibrating uniformly in water, the inertia force is given by

$$
f_{j}=\left(m_{t}+\rho \pi D^{2} \ell / 4\right) \omega^{2} d \sin (\omega t)
$$

where $\mathrm{mt}$ is tube mass, $\ell$ is tube length, $\rho$ is water density, $\omega$ is oscillation frequency $(\mathrm{rad} / \mathrm{sec})$, and $\mathrm{d}$ is oscillation peak amplitude. In testing, the inertia force $\mathrm{f}_{\mathrm{j}}$ is measured for a given excitation $\omega$ and vibration amplitude $d$. In the calibration, $\omega$ varies from $\approx 0.25 \mathrm{~Hz}$ to $2.0 \mathrm{~Hz}$ and d varies from 0.5 to $5.0 \mathrm{~mm}$. From Eq. 10, the ratio of $f_{j} / d$ (or $g_{j} / d$ ) is proportional to $\omega^{2}$. Figure 5 shows $g_{j} / d$ as a function of excitation frequency for typical cases in which a single tube oscillates in water in the $\mathrm{x}$ and $\mathrm{y}$ directions. From the data, a power-law curve is used to determine the constant. Theoretically, the ratio $\mathrm{f}_{\mathrm{j}} / \mathrm{d}$ (or $\mathrm{g}_{\mathrm{j}} / \mathrm{d}$ ) should be proportional to $\omega^{2}$; the actual power is very close to 2.0. All force transducers are calibrated in two directions. The results of the calibration are given in Table 1. 


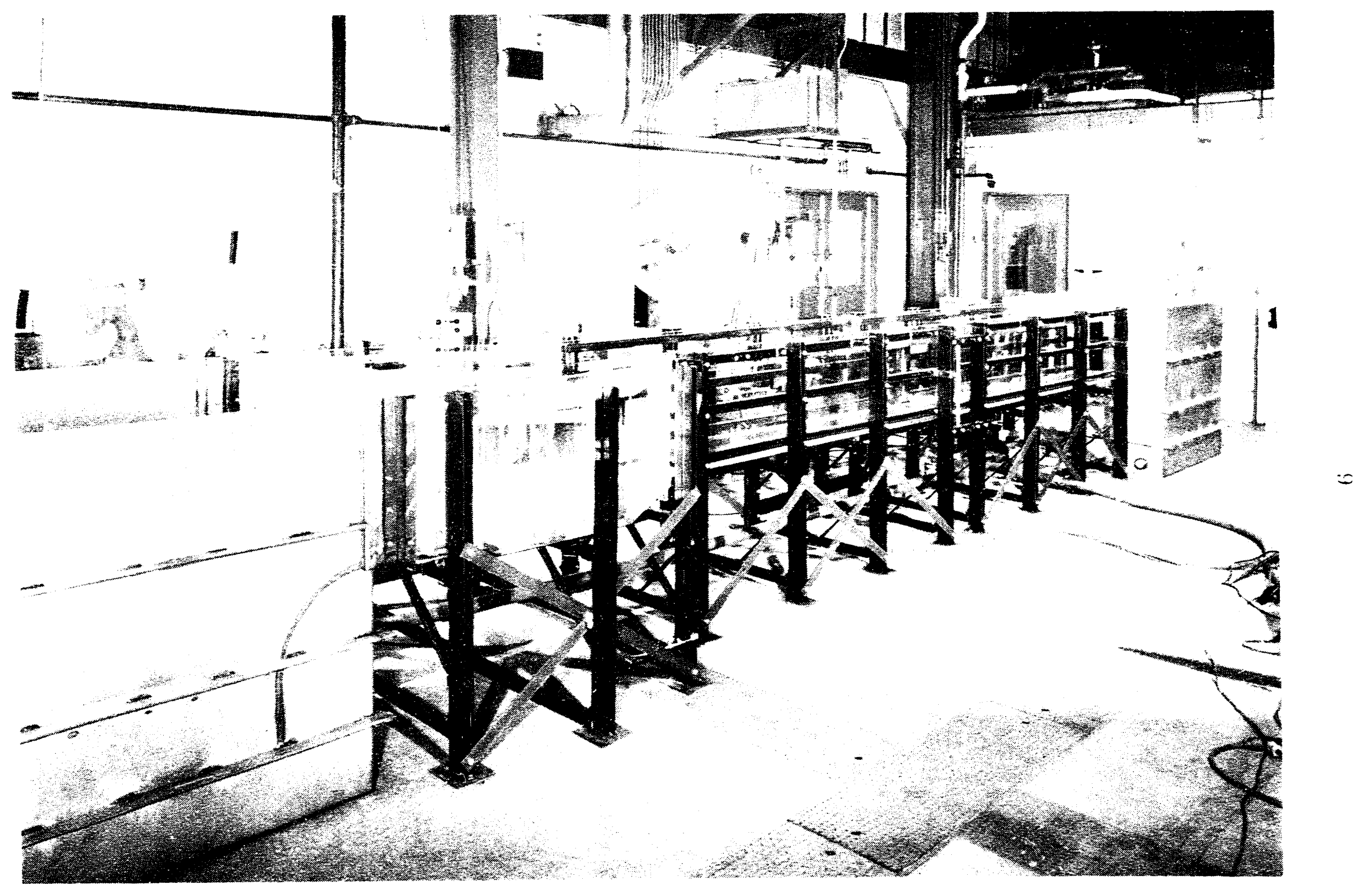

Fig. 3. Water channel 


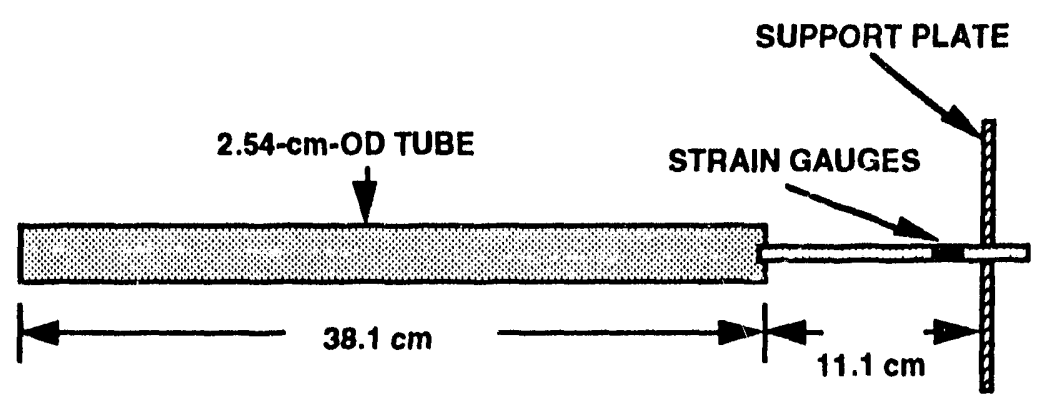

(a)

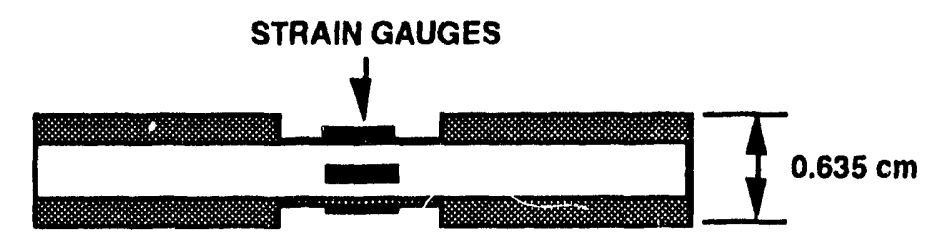

(b)
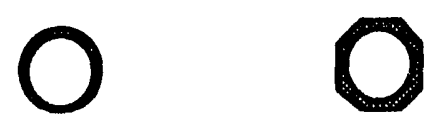

(c)

Fig. 4. Schematic representation of force transducer: (a) force transducer, (b) flexible tube, (c) cross section of flexible tube for strain gauges,

Table 1. Calibration constants for three circular-cross-section and three hexagonal-cross-section tubes

\begin{tabular}{cccc}
\hline Tube Cross Section & Tube Number & $\begin{array}{c}\text { x Direction } \\
(\mathrm{g} / \mathrm{v})\end{array}$ & $\begin{array}{c}\text { y Direction } \\
(\mathrm{g} / \mathrm{v})\end{array}$ \\
\hline Circular & 1 & 4.325 & 4.246 \\
Circular & 2 & 7.643 & 7.631 \\
Circular & 3 & 6.244 & 5.646 \\
Hexagonal & 1 & 2.770 & 2.597 \\
Hexagonal & 2 & 2.266 & 2.258 \\
Hexagonal & 3 & 2.059 & 2.173 \\
\hline
\end{tabular}

\subsection{Test Section}

An array of tubes is assembled in the test area. Figure 6 shows several tube arrangements: tube row, square array, and triangular array. An array of tubes, 

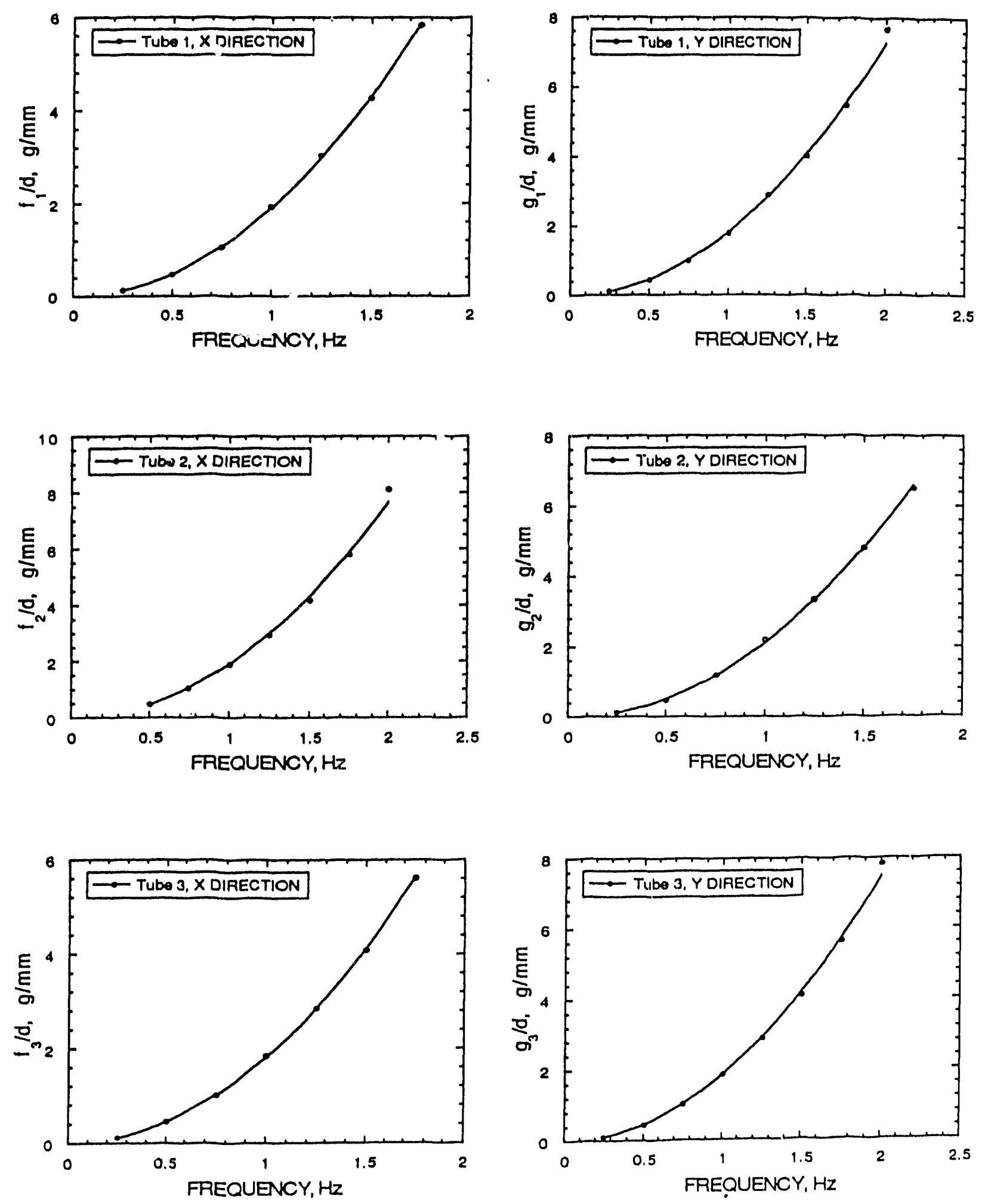

Fig. 5. Force-to-displacement ratio as a function of excitation frequency for typical cases in which a single tube is oscillating in water 


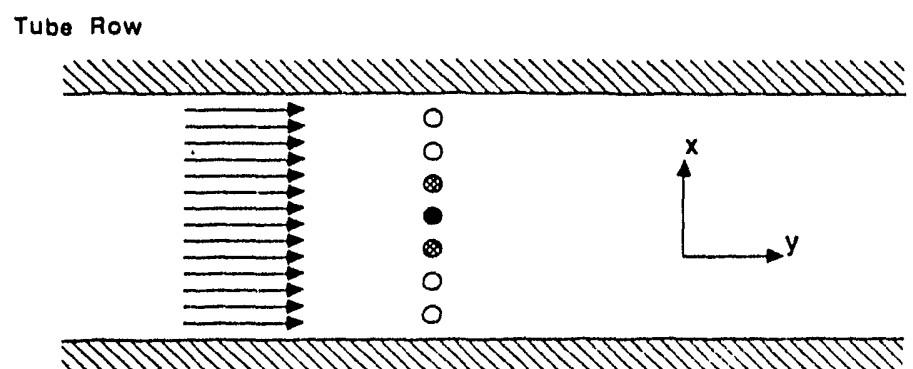

Rectangular Array

S ll
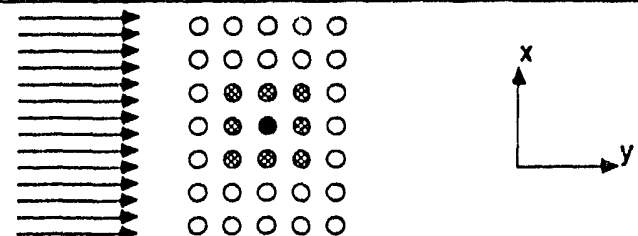

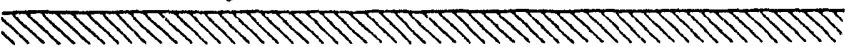

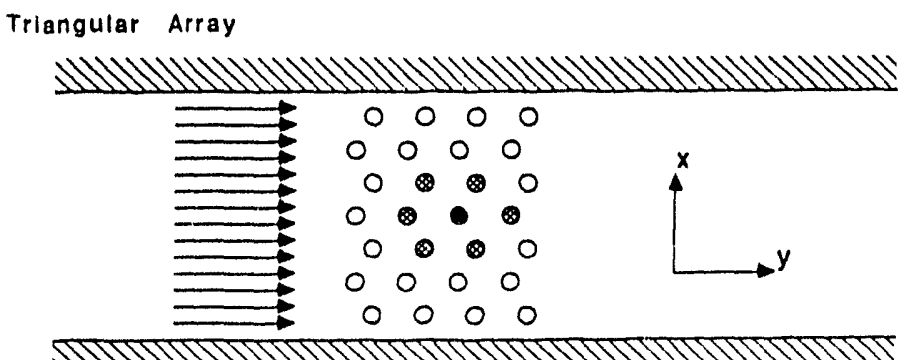

O Rigid Tube

Q Force Transducer

- Excitation Tubo

Fig. 6. Tube row, square array, and triangular array

denoted by solid and shaded circles, is active, while the others, denoted by open circles, are dummy tubes. All tubes except the middle one, denoted as Tube 1 by a solid circle, are clamped to a support plate with a nut attached to a smaller supporting tube. Tube 1 is not attached to the support plate, but passes through a circular hole in the support plate and is connected to an electromagnetic shaker. The shaker provides the support for Tube 1. In addition, specific oscillations can be assigned to Tube 1 in the $\mathrm{x}$ or $\mathrm{y}$ direction, and both the oscillation amplitude and frequency of the shaker can be controlled in the appropriate range.

During tests, the water surface is kept at such a leves that the active length of the tubes is submerged in the flow. Normally, only a small portion of the supporting tube (less than $1.3 \mathrm{~cm}$ ) is submerged in water. Therefore, the strain gauges do not require waterproofing. 


\section{Test Procedures and Data Analysis}

A flow diagram of the instrumentation and exciter is shown in Fig. 7. The exciter provides sinusoidal displacement at a frequency varying from $\approx 0.02 \mathrm{~Hz}$ to $2.0 \mathrm{~Hz}$. Displacement and force signals are first filtered by band-pass filters to eliminate low- and high-frequency noises and then digitized and stored in the analyzer. These signals are analyzed to obtain the oscillation displacement of the tube, the magnitudes of the forces acting on the active tubes, and the phase between the motion-dependent fluid force and tube displacement.

In Fig. 7, the test facility, instrumentation, and data analysis systems are shown ready for a series of tests. The first tube array tested was a row of tubes with a pitch-to-diameter ratio (T/D, where $\mathrm{T}$ is the gap) of 1.35 (Fig. 8). Motiondependent fluid forces were measured for Active Tubes 1, 2, and 3, with Tube 1 oscillating in the lift $(x)$ or drag $(y)$ direction. A photograph of the setup is shown in Fig. 9.

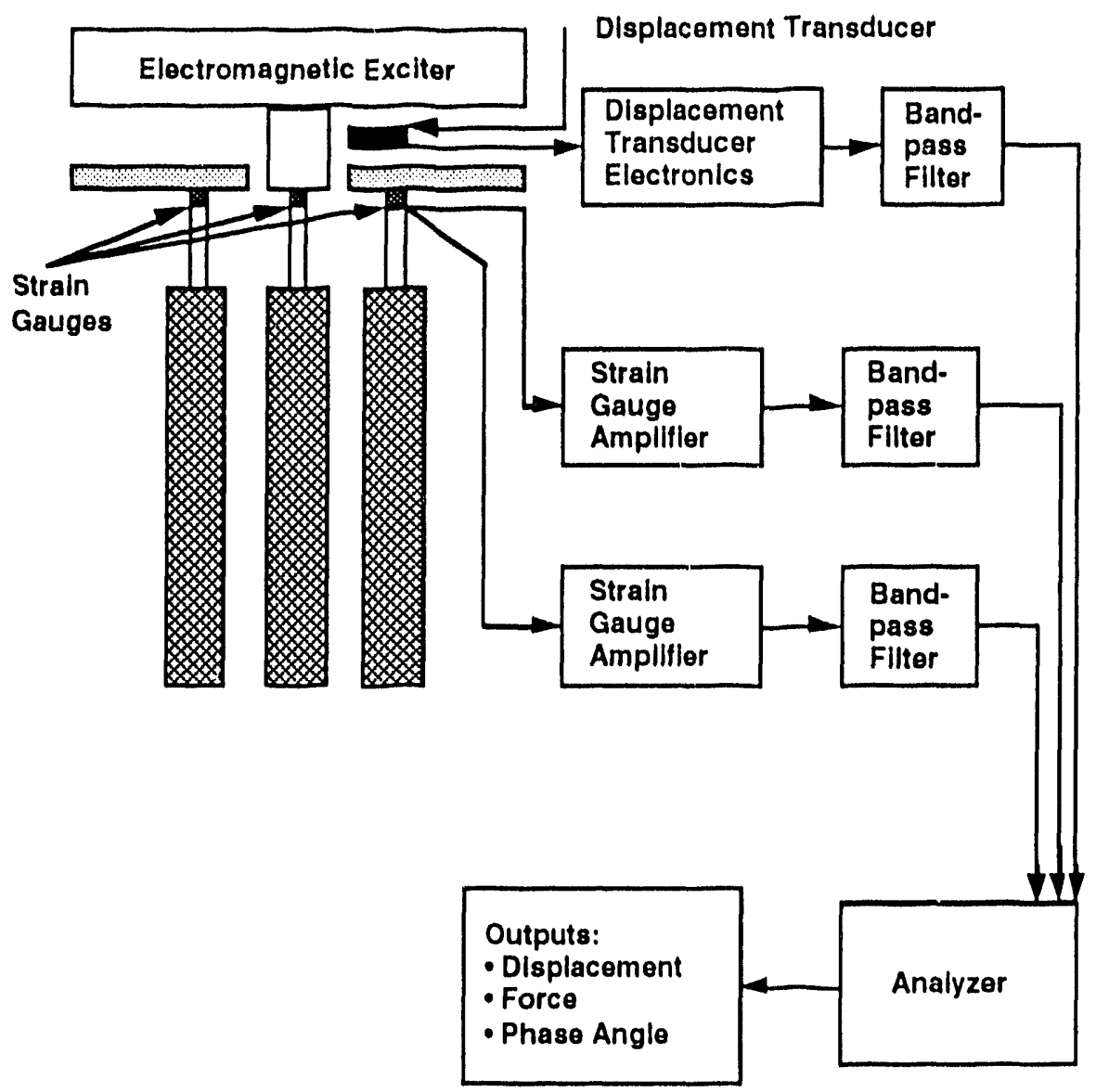

Fig. 7. Flow diagram of instrumentation for data analysis 


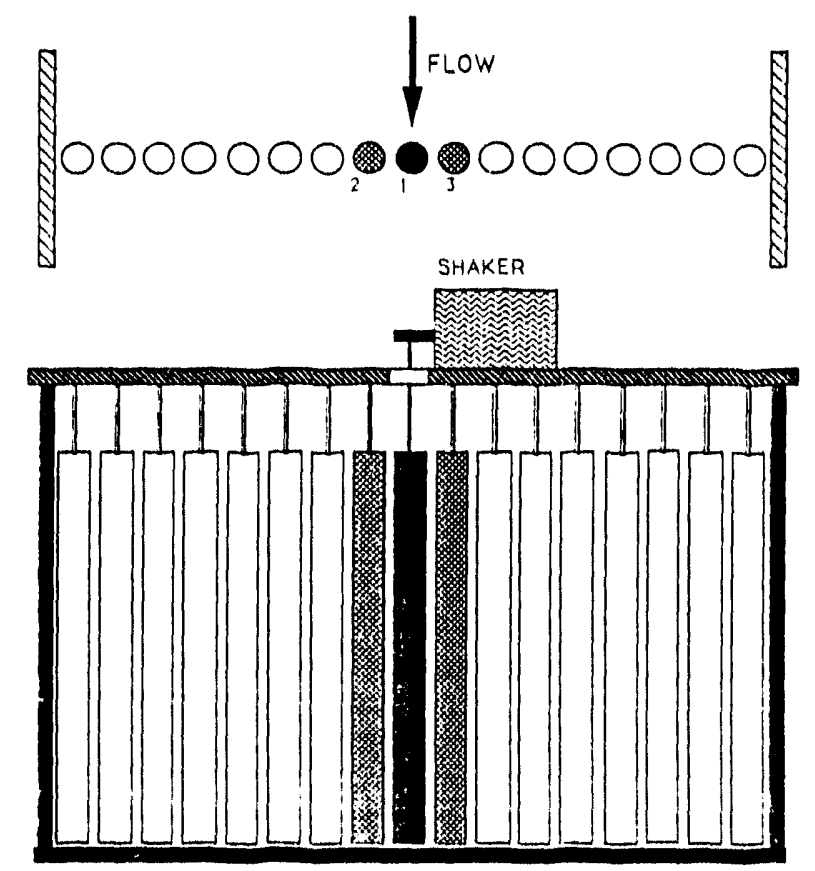

Fig. 8. Schematic representation of fïrst tube array tested, i.e., row of tubes in ( ossflow

\section{Test Results}

\subsection{Added Mass Coefficients}

The added mass coefficients for a row of tubes were measured as a function of excitation amplitude and frequency. Typical results are shown in Figs. 10a and b, which show the ratio of measured force to tube displacement as a function of tube displacement for two frequencies, $0.5 \mathrm{~Hz}$ and $1.0 \mathrm{~Hz}$. Tube 1 was excited in the $\mathrm{x}$ direction, and the forces acting on Tubes $1-3$ in the $x$ direction were measured. The amplitude ratios for the three tubes were independent of the excitation amplitude and the forces acting on Tubes 2 and 3 were equal.

The calculation of the added mass coefficients is based on the measured forces. The results are given in Table 2 for excitation at $0.5 \mathrm{~Hz}$.

The added mass coefficients were rasasured for a series of excitation amplitudes, ranging from $\approx 0.8$ to $5 \mathrm{~mm}$, which correspond to $\approx 10-60 \%$ of the gap between the tubes. The effects of vibration amplitude on the added mass coefficients in this range of motion are not significant. The added mass coefficients were also measured for various excitation frequencies. For example, the values for the added mass coefficient $\alpha_{11}$, obtained for three excitation frequencies, $0.5,1.0$, 

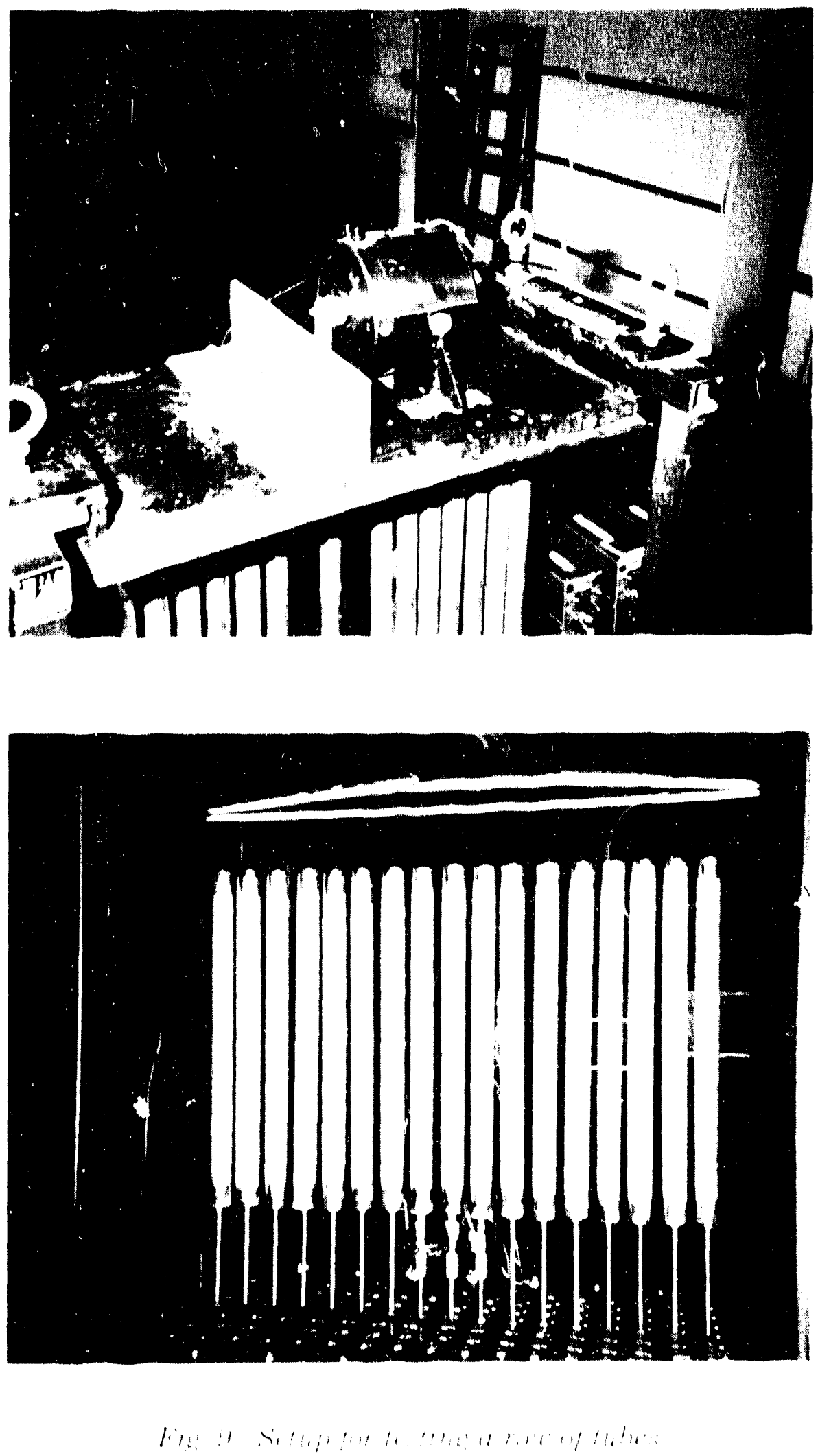

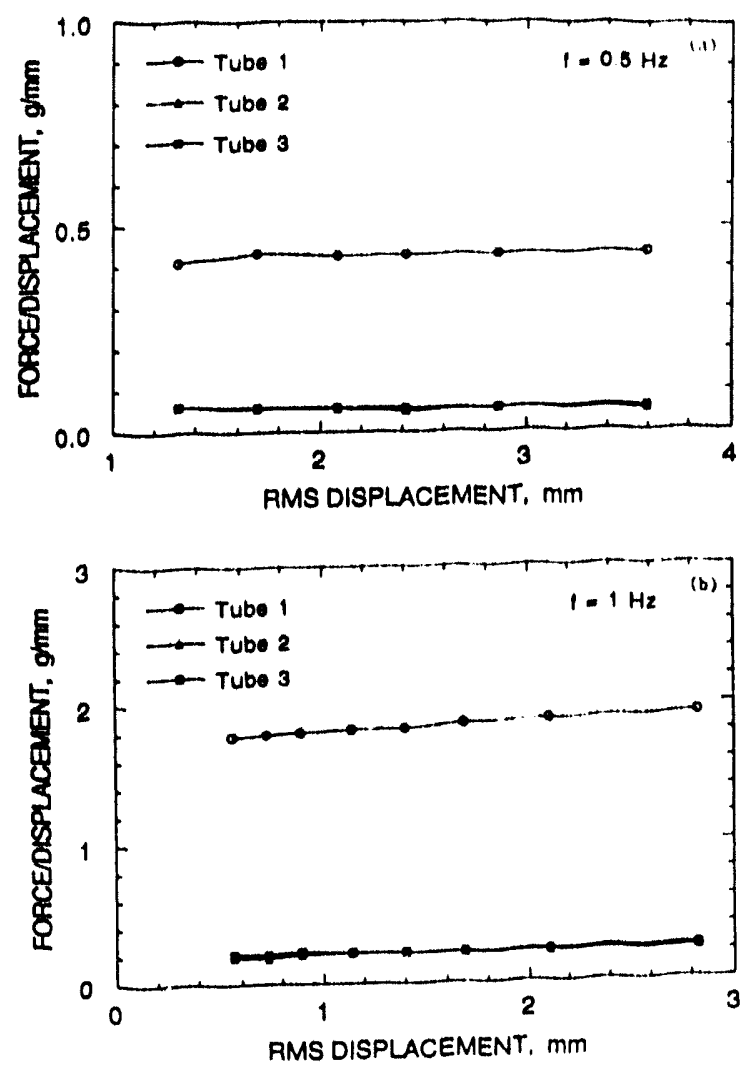

Fig. 10. Force-to-displacement ratio as a function of RMS displacement when frequency is (a) 0.5 and (b) $1 \mathrm{~Hz}$

Table 2. Experimental and theoretical values of added mass coefficients with excitation at $0.5 \mathrm{~Hz}$

\begin{tabular}{ccc}
\hline $\begin{array}{c}\text { Added Mass } \\
\text { Coefficients }\end{array}$ & $\begin{array}{c}\text { Theoretical Results } \\
\text { of Chen 1975 }\end{array}$ & $\begin{array}{c}\text { Experimental } \\
\text { Data }\end{array}$ \\
\hline$\alpha_{11}$ & 1.113 & 1.098 \\
$\alpha_{12}$ & -0.282 & -0.282 \\
$\alpha_{13}$ & -0.282 & -0.298 \\
$\beta_{11}$ & 1.114 & 1.089 \\
$\beta_{12}$ & 0.340 & 0.336 \\
$\beta_{13}$ & 0.340 & 0.336 \\
\hline
\end{tabular}


and $1.5 \mathrm{~Hz}$, were $1.098,1.122$, and 1.144 , respectively. The experimental data confirm two characteristics: (1) at low excitation amplitude, the linear flow theory, based on the potential flow theory, will provide added mass coefficients with sufficient accuracy. (2) at high kinetic Reynolds number (which is equal to the circular frequency of oscillation multiplied by tube diameter squared and fluid kinetic viscosity, Chen $1987 \mathrm{a}$ ), the oscillation amplitude is insignificant.

\subsection{Vortex Shedding}

One of the main excitation sources for tube vibration in crossflow is vortex shedding. For a tube row in crossflow, vortex shedding is important. Tests are conducted for the tube row by measuring the resultant lift and drag forces acting on the three active tubes at different flow velocities. From the frequency spectra of the fluid excitation forces in the lift direction, the vortex shedding frequencies can be identified and the Strouhal number St can be calculated:

$$
S t=f D / U,
$$

where $f$ represents the frequencies corresponding to the peaks in the power spectra of lift forces. In the drag direction, the frequencies are twice those of the lift direction; therefore, from the frequencies corresponding to the peaks in the power spectra of drag forces,

$$
\mathrm{St}=\mathrm{fD} / 2 \mathrm{U} \text {. }
$$

Based on the power spectra of the lift forces and drag forces at different flow velocities, three Strouhal numbers were calculated: $0.105,0.22$ and 0.43 . The detailed flow field across the tube row was not studied in this test. Differing vortex shedding frequencies are associated with differing flow patterns for steady flow across a tube row.

Many studies have focused on the vortex shedding process for a single tube in crossflow (Chen 1987a). The Strouhal number is a function of the Reynolds number and in the subcritical region, it is equal to $\approx 0.2$. In this test, Reynolds number varies from $\approx 2500$ to 3800 . It is in the subcritical region. Various investigators have found different vortex shedding frequencies for tube rows in crossflow (Chen 1968; Borges 1969; Clasen and Gregorig 1971; Ishigai, Nishikawa, and Yagi 1973; Auger and Coutanceau 1978). Figure 11 shows results obtained in the present study and published data, as a function of the pitch-to-diameter ratio (T/D) of tube rows. The three values obtained during this study compare reasonably well with the published data. 


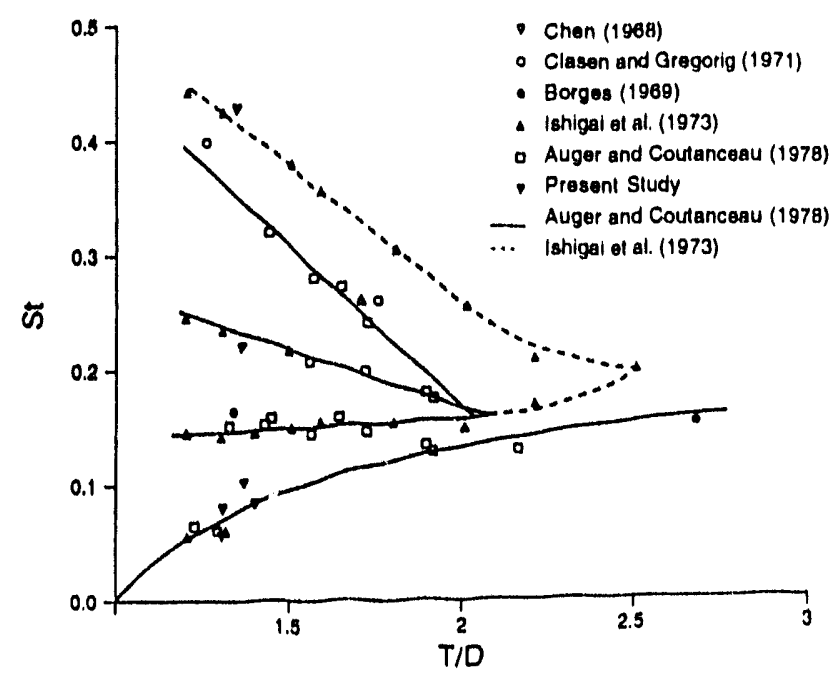

Fig. 11. Strouhal number for tube row as a function of pitch-to. diameter ratio

Vortex shedding for a flow across a tube array is fairly complicated. In this study, the vortex shedding frequencies were determined from the frequency spectra of the resultant lift and drag forces and not from the direct measurements of flow velocity or fluid pressure. Figure 11 show/s that there are five vortex shedding frequencies for a tube row with $T / D=1.35$. Two vortex shedding frequencies that can be measured in the wake do not appear in the resultant forces acting on the tubes. To understand the detailed vortex shedding process, direct measurements of local flow velocity and fluid pressure behind the tube row, as well as visualization of flow patterns, provides a better understanding. Measurements of resultant fluid forces are useful for predicting tube response in crossflow.

\subsection{Motion-Dependent Fluid Forces}

Fluid forces on the tubes are relatively small except for the force on the tube being excited by the shaker. The magnitude of the forces depends on the excitation amplitude, excitation frequency, and flow velocity. Figure 12 shows fluid force components $f_{1}, f_{2}, g_{1}, g_{2}$, acting on Tubes 1 and 2 as a result of the motion of Tube 1 in the $y$ direction, $\mathrm{v}_{1}$. The flow velocity was $0.15 \mathrm{~m} / \mathrm{s}$ and the excitation frequency to Tube 1 was $U .15 \mathrm{~Hz}$. The signals presented in Fig. 12 have been filtered. The dominant frequency of fluid forces is the same as the excitation frequency; however, the fluid force componsnts are not in phase with the tube displacement $v_{1}$. Depending on the phase between the tube displacement and fluid forces, the fluid forces may be an excitation mechanism or energy dissipation mechanism. 


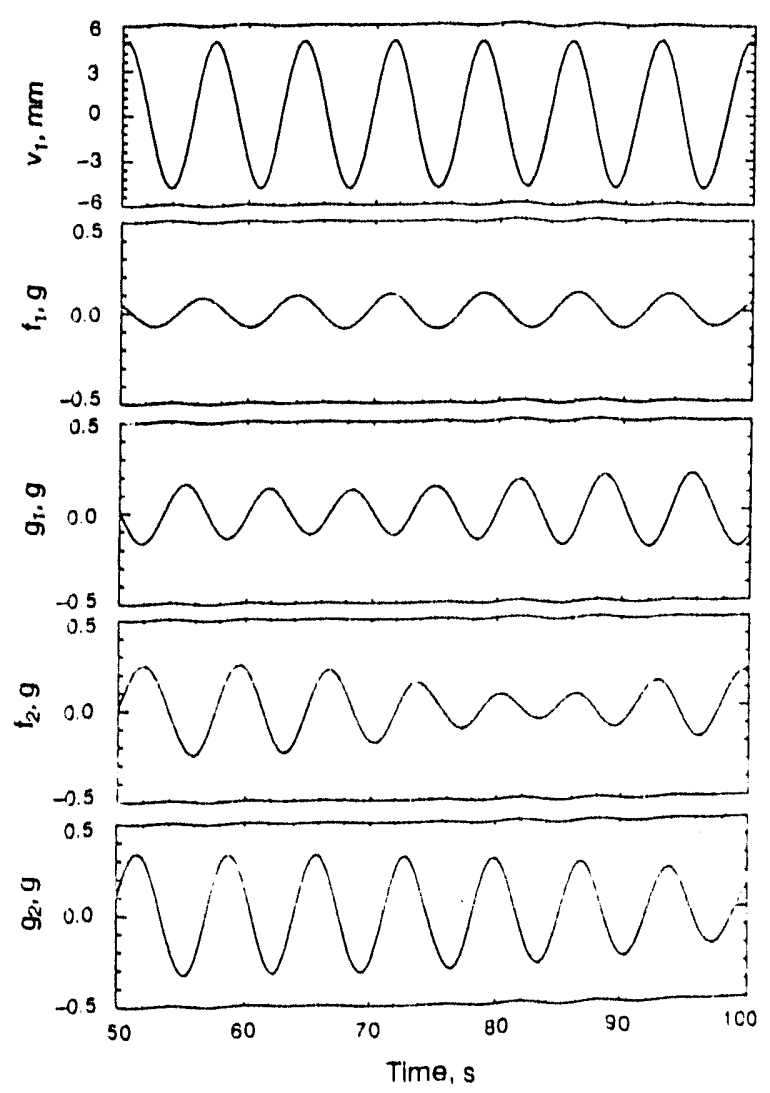

Fig. 12. Fluid-force components acting on tube row

Figure 13 shows the loci of the unsteady fluid forces $g_{1}$ at excitation frequencies of 0.15 and $2 \mathrm{~Hz}$. At $0.15 \mathrm{~Hz}$, the component $g_{1}$ is negative when the displacement is positive and vice versa; therefore, it acts as an excitation force. At $2.0 \mathrm{~Hz}$, the component $g_{1}$ is positive when the displacement is positive; therefore, it acts as a damping force. A damping force will contribute to energy dissipation, which will reduce tube response, whereas an excitation force will contribute to negative damping, which may result in fluidelastic instability.

The phase angle is defined as the angle between the fluid-force component and the tube displacement. This angle can be obtained from the correlation between the two time histories of fluid force and tube displacement, or from the time history plots given in Fig. 12. Figure 14 shows typical magnitude fluid forces (RMS values) and phase angics as a function of reduced flow velocity. When the reduced flow is small, $\approx<10$, the fluid forces decrease rapidly with increasing reduced flow velocity. This is attributed to the effect of the inertia force of added mass of fluid. At higher reduced flow velocity, the magnitude of the fluid force is almost independent of the reduced flow velocity. Similar trends are noted for the phase angle. At low reduced flow velocity, the phase angle varies more dramatically with reduced flow velocity, 

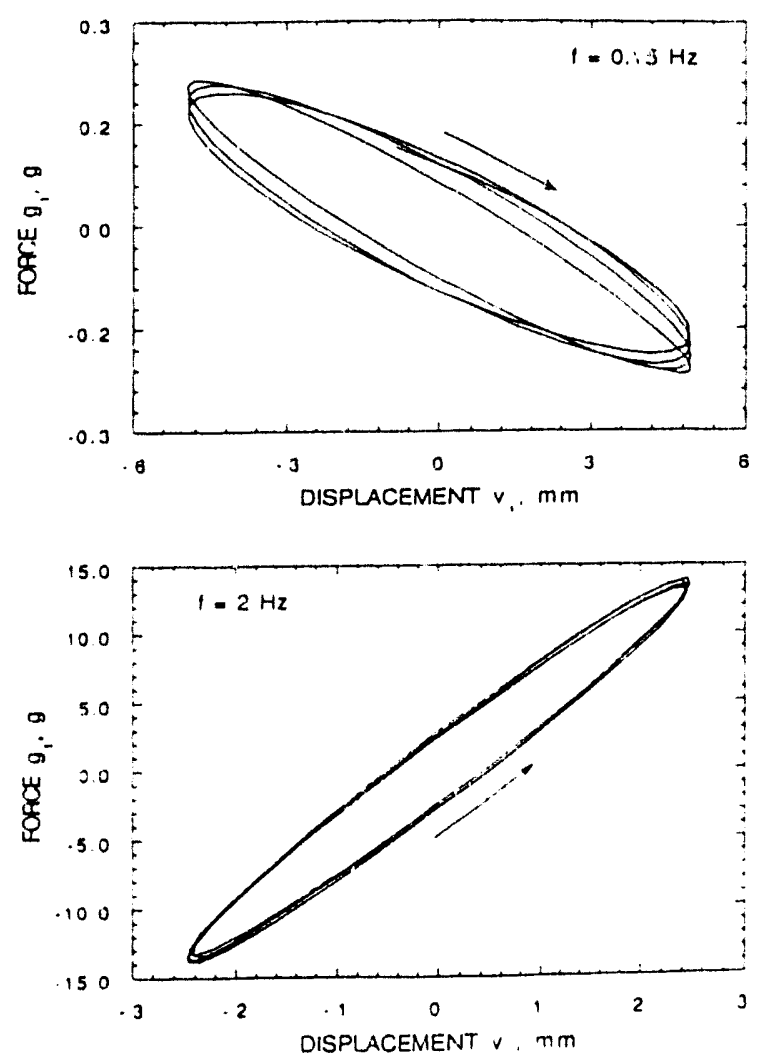

Fig. 13. Fluid force $g_{1}$ as a function of tube displacement $v_{1}$ for frequencies of 0.15 and $2 \mathrm{~Hz}$

while at large reduced flow velocity, the variation of the phase angle with reduced flow velocity is much smaller.

\subsection{Fluid-Force Coefficients}

The critical elements for predicting fluidelastic instability of tube arrays in crossflow are motion-dependent fluid-force coefficients. A reliable method to obtain these coefficients will contribute significantly to the development of accurats prediction techniques. From the motion-dependent fluid forces acting on the exciting tube itself and the surrounding tubes and their phase angles with respect io the displacement of the excitation tube, as well as the added mass coefficients based on the potential flow theory, fluid-damping and fluid-stiffness coefficients can be calculated by the method described in Section 3. Several series of tests were performed for the tube row with a pitch-to-diameter ratio of 1.35 with Type $A$ and $B$ force transducers. The excitation amplitude was set at different values by controlling the voltage at the shaker. The displacement of the excited tube depends 

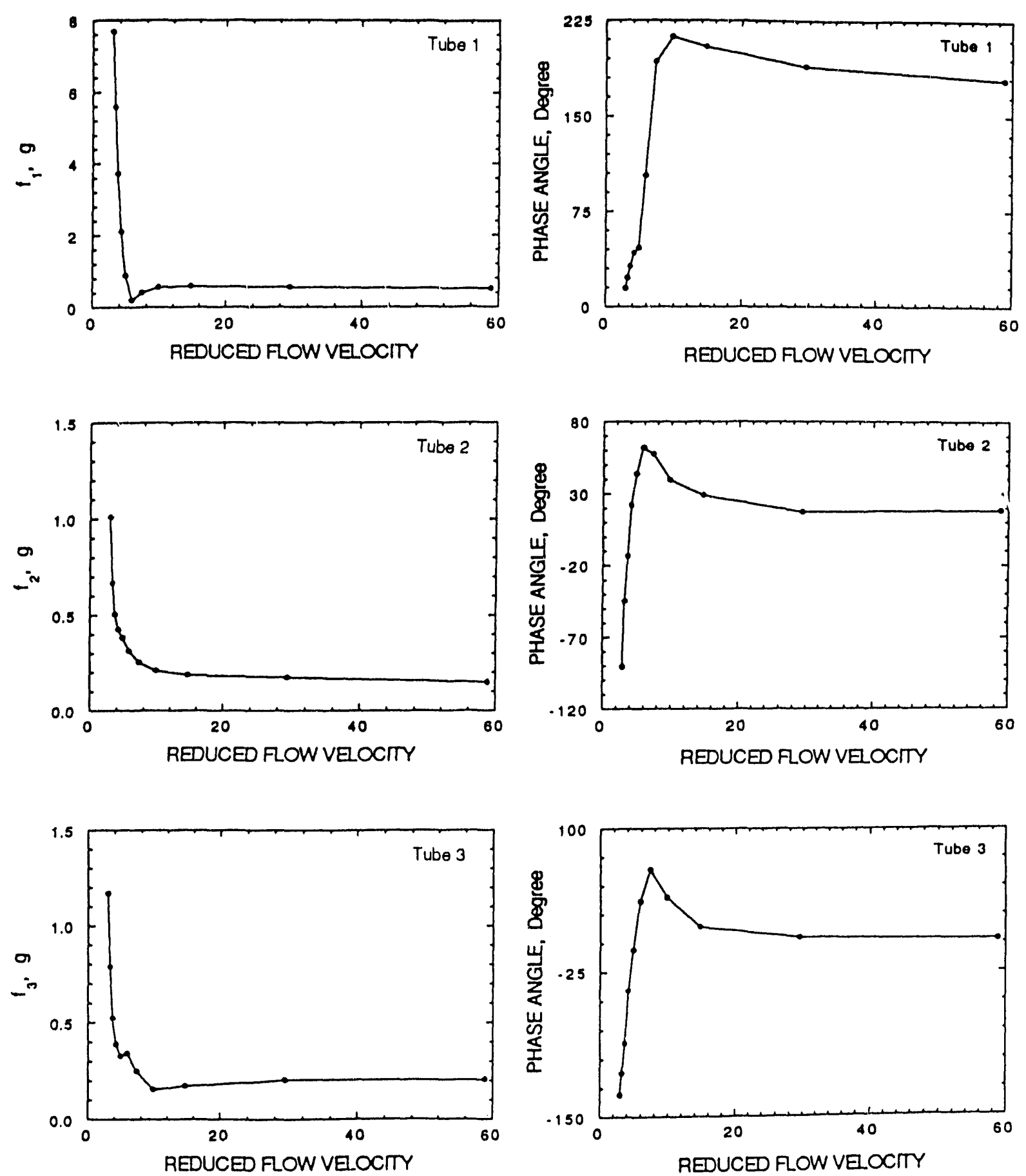

(a)

Fig. 14. Magnitude of fluid force and phase angle between fluid force and tube displacement $u_{1}$ due to oscillation of Tube 1 in (a) the $x$ and (b) the $y$ directions 

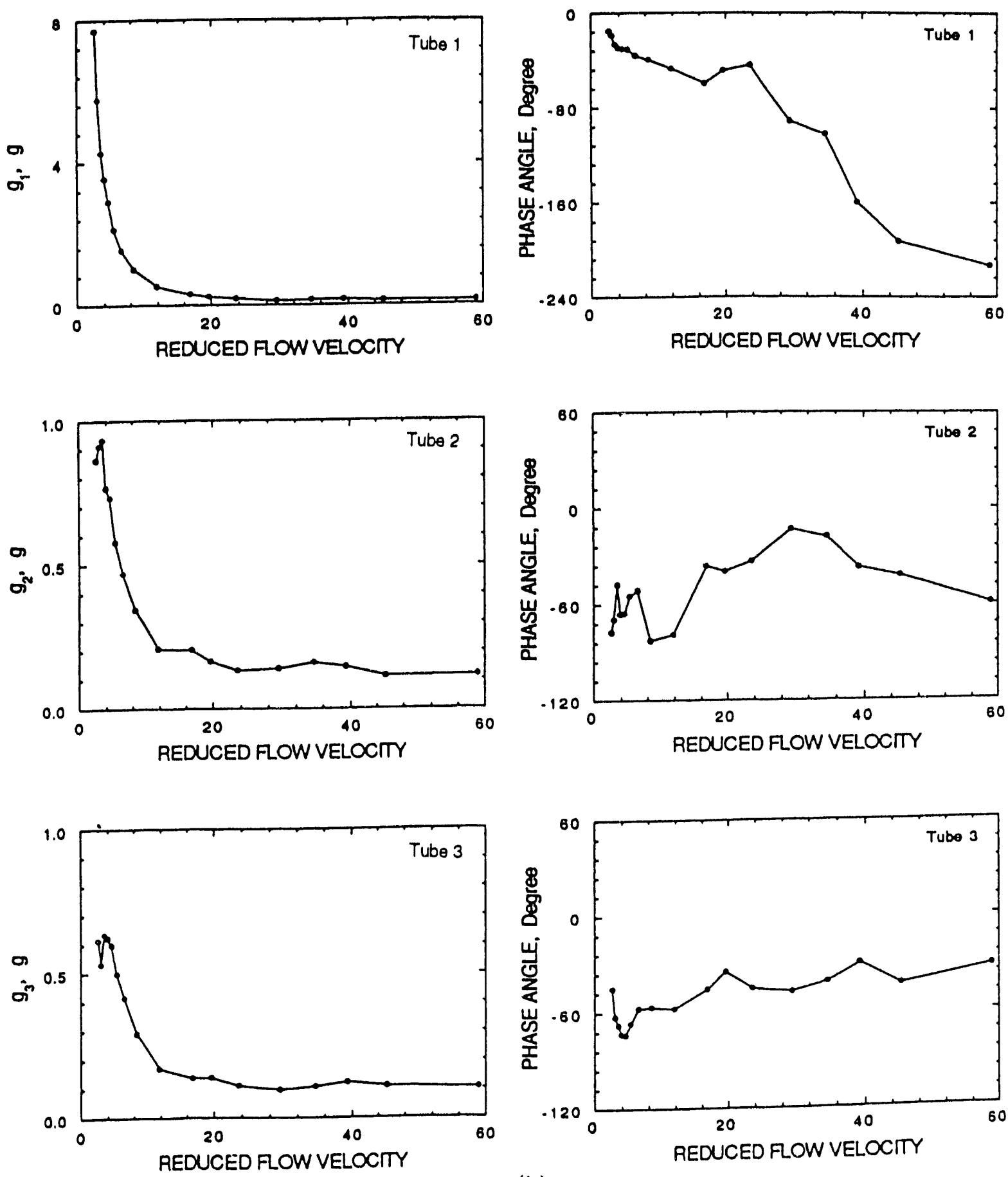

(b)

Fig. 14. (Cont'd) 
on the excitation frequency. For example, at $0.1 \mathrm{~m} / \mathrm{s}$, the excited RMS tube displacements with an excitation force denoted by $1.5 \mathrm{v}$ vary from $2.65 \mathrm{~mm}$ at $0.1 \mathrm{~Hz}$ to $1.68 \mathrm{~mm}$ at $2 \mathrm{~Hz}$.

Type-A force transducers: Fluid forces were measured for three flow velocities, with the reduced flow velocity varying from $\approx 2$ to 60 for excitation in the $\mathrm{x}$ direction. Only one flow velocity was used in the $y$ direction. The results are given in Figs. 15-18.

Type-B force transducers: The flow velocity is set at $0.1 \mathrm{~m} / \mathrm{s}$. Two series of tests were performed with Type-B force transducers:

- The excitation in the $\mathrm{x}$ direction was given at three excitation levels and the reduced llow velocity varied from $\approx 2$ to 160 . In the y direction, the excitation was given at five excitation levels and the reduced llow velocity varied from $\approx 2$ to 40 . The results are given in Figs. 19-22.

- The reduced flow velocity varied from $\approx 2$ to 200 . The excitation was set at two different levels. The results are given in Figs. 23-26.

Several interesting characteristics of the fluid-force coefficients were noted.

- At high reduced flow velocity, all fluid-force coefficients are approximately constant. In this range, fluid-force coefficients obtained at a particular reduced flow velocity are applicable for all values of reduced flow velocity. This characteristic was also noted for structural components with other shapes (Chen 1987b).

- Fluid-force coefficients obtained at different flow velocities are approximately the same. Therefore, for relatively small-amplitude oscillations, fluid-force coefficients are a function of reduced flow velocity only. In the measurement of motion-dependent fluid forces, an appropriate combination of flow velocity and oscillation frequency can be utilized to obtain the best results.

- At small reduced flow velocity, all fluid-force coefficients are a function of reduced flow velocity and the fluid-force coefficients must be measured for all values of reduced flow velocity. In this range of reduced flow velocity, the functional dependence of fluid force coefficients with reduced flow velocity is difficult to characterize. 

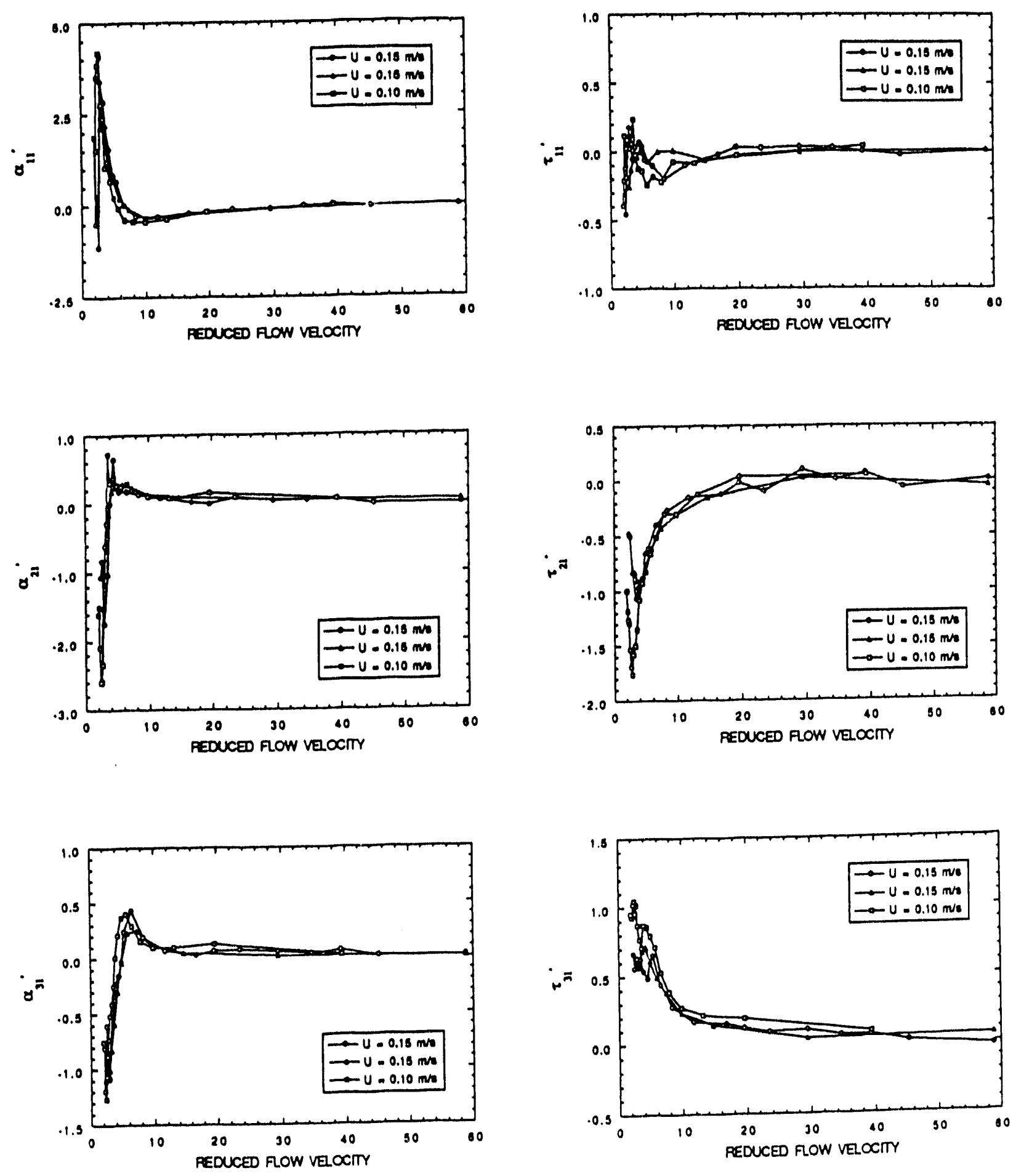

Fig. 15. Fluid-damping coefficients $\alpha_{11}^{\prime}, \quad \alpha_{21}^{\prime}, \alpha_{31}^{\prime}, \tau_{11}^{\prime}, \tau_{21}^{\prime}$, and $\tau_{31}^{\prime}$, force transducer Type A 

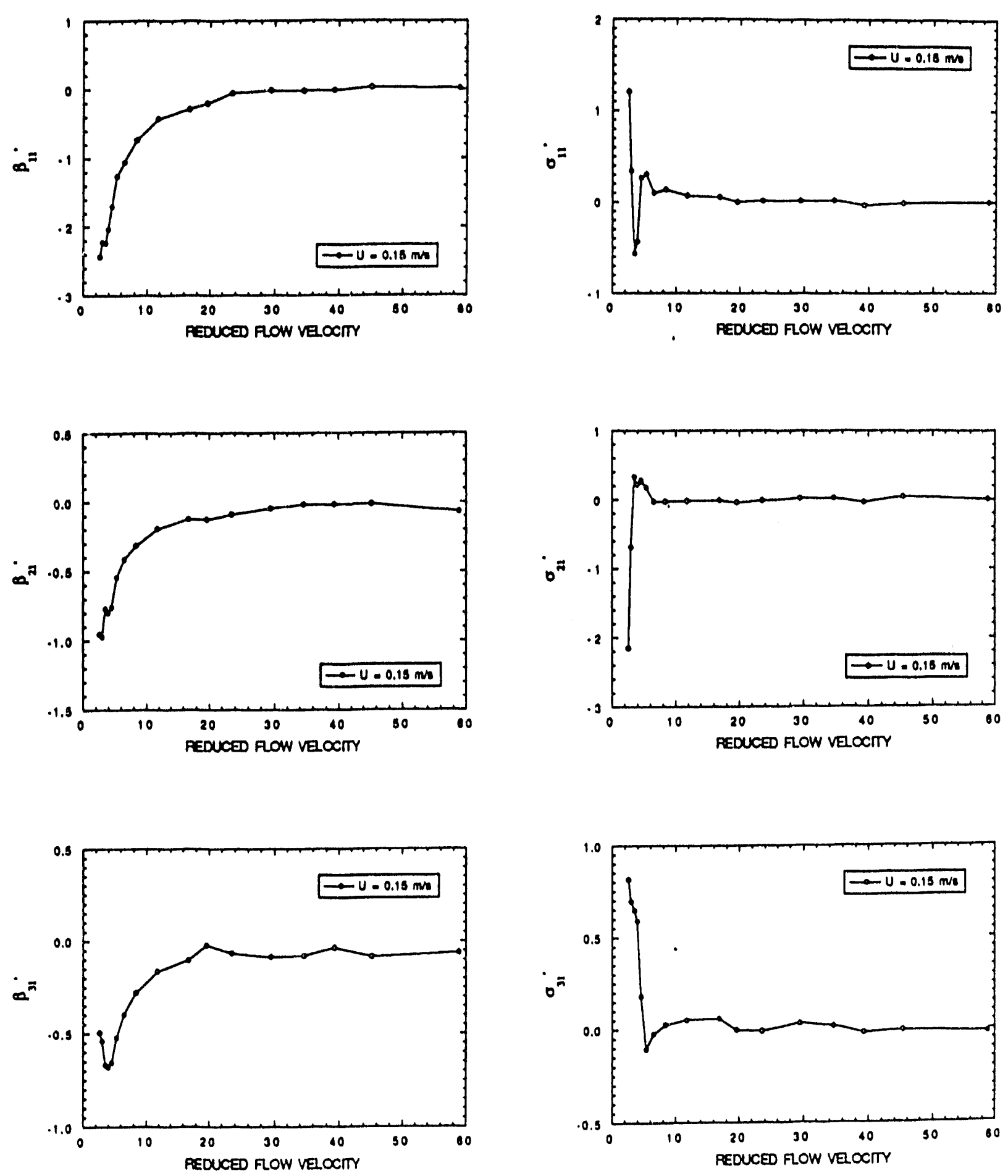

Fig. 16. Fluid-damping coefficients $\beta_{11}^{\prime}, \beta_{21}^{\prime}, \beta_{31}^{\prime}, \sigma_{11}^{\prime}, \sigma_{21}^{\prime}$, and $\sigma_{31}^{\prime}$, force iransducer Type A 

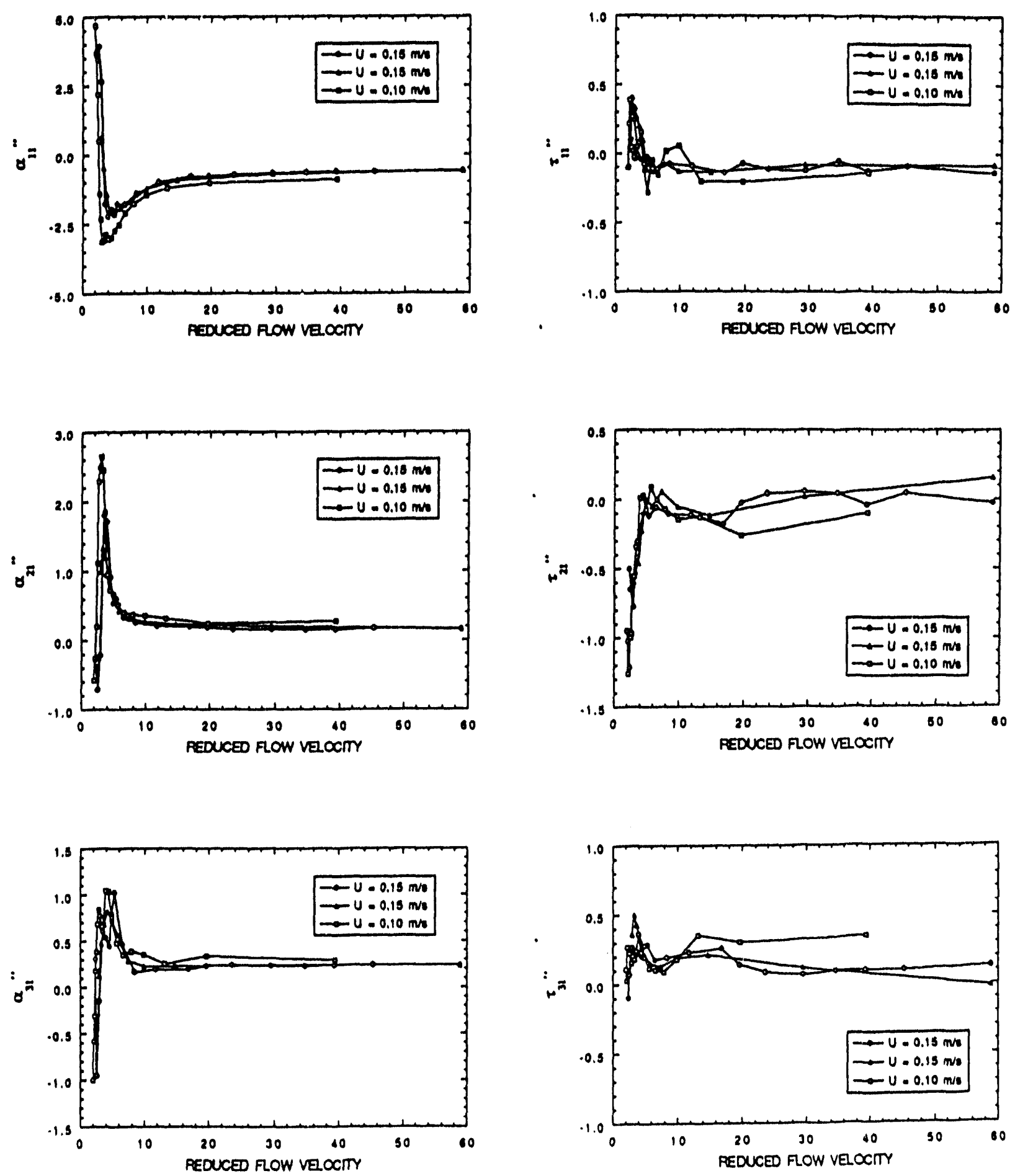

Fig. 17. Fluid-stiffness coefficients $\alpha_{11}^{\prime \prime}, \alpha_{21}^{\prime \prime}, \alpha_{31}^{\prime \prime}, \tau_{11}^{\prime \prime}, \tau_{21}^{\prime \prime}$, and $\tau_{31}^{\prime \prime}$, force transducer Type A 

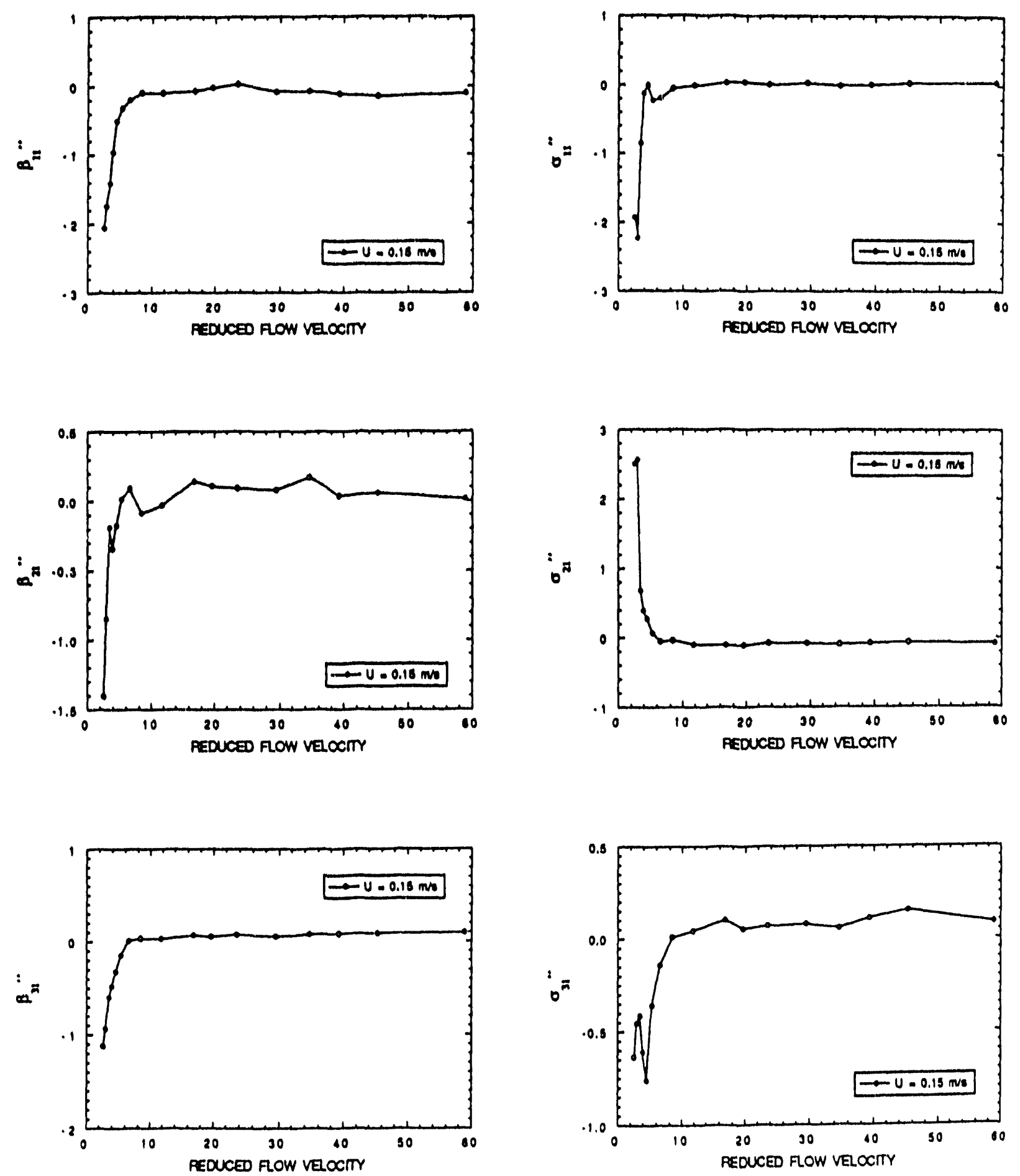

Fig. 18. Fluid-stiffness coefficients $\beta_{11}^{\prime \prime}, \beta_{21}^{\prime \prime}, \beta_{31}^{\prime \prime}, \sigma_{11}^{\prime \prime}, \sigma_{21}^{\prime \prime}$, and $\sigma_{31}^{\prime \prime}$, force transducer Type A 

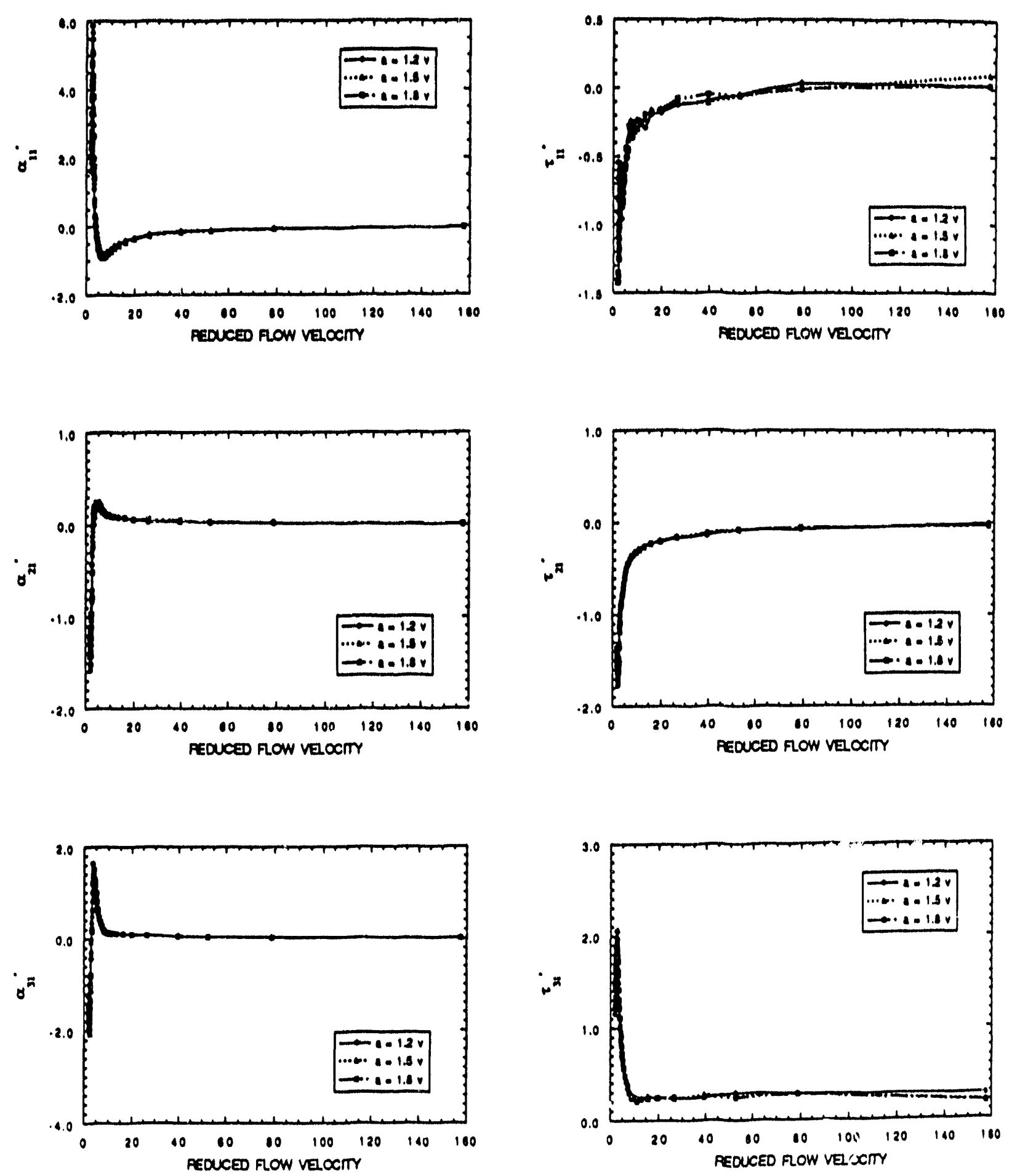

Fig. 19. Fluid-damping coefficients $\alpha_{11}^{\prime}, \alpha_{21}^{\prime}, \alpha_{31}^{\prime}, \tau_{11}^{\prime}, \tau_{21}^{\prime}$, and $\tau_{31}^{\prime}$, force transducer Type B, reduced flow velocity from 2 to 160 

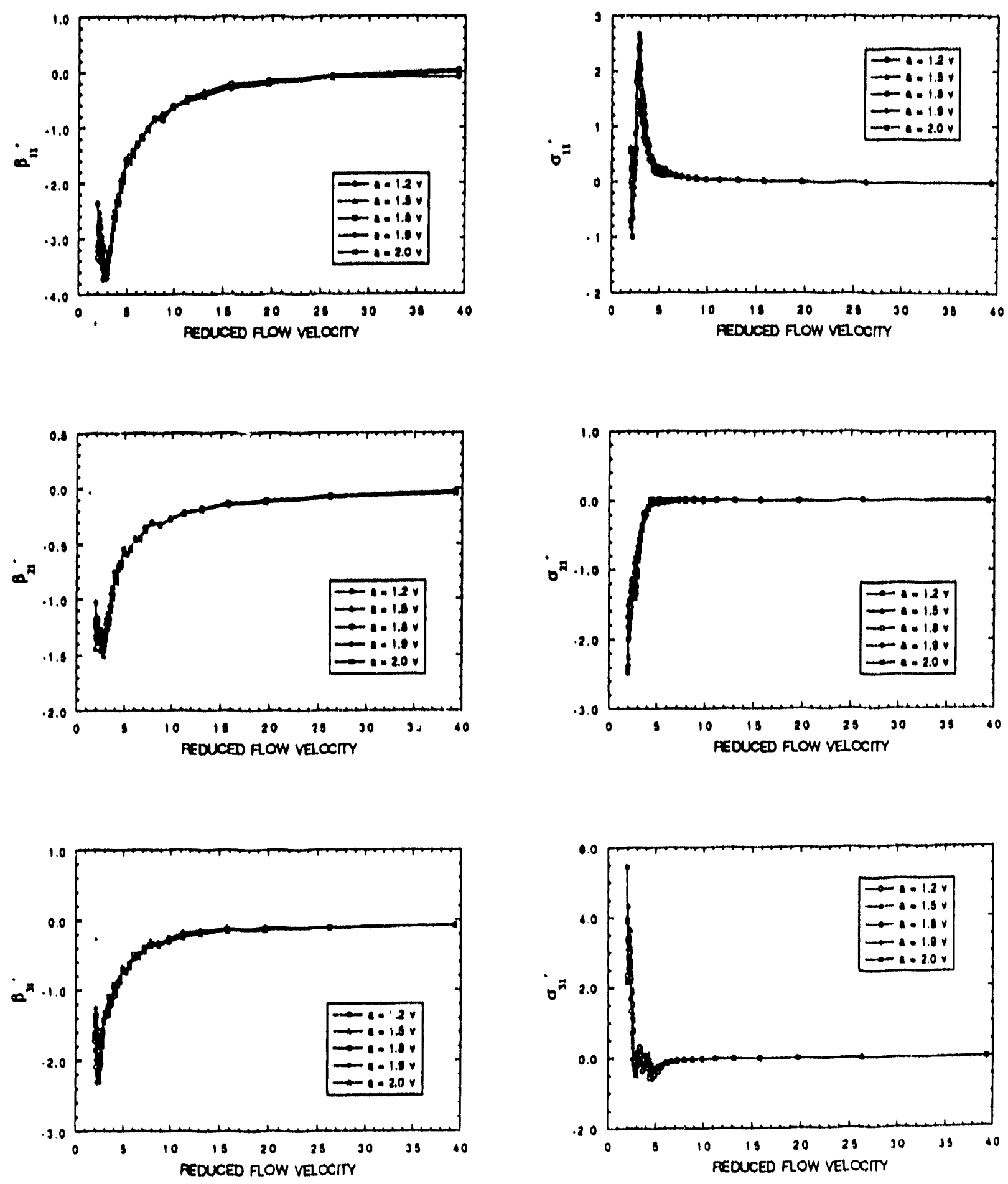

Fig. 20. Fluid-damping coefficients $\beta_{11}^{\prime}, \beta_{21}^{\prime}, \beta_{31}^{\prime}, \sigma_{11}^{\prime}, \sigma_{21}^{\prime}$, and $\sigma_{31}^{\prime}$, force transducer Type $B$, reduced flow velocity from 2 to 40 

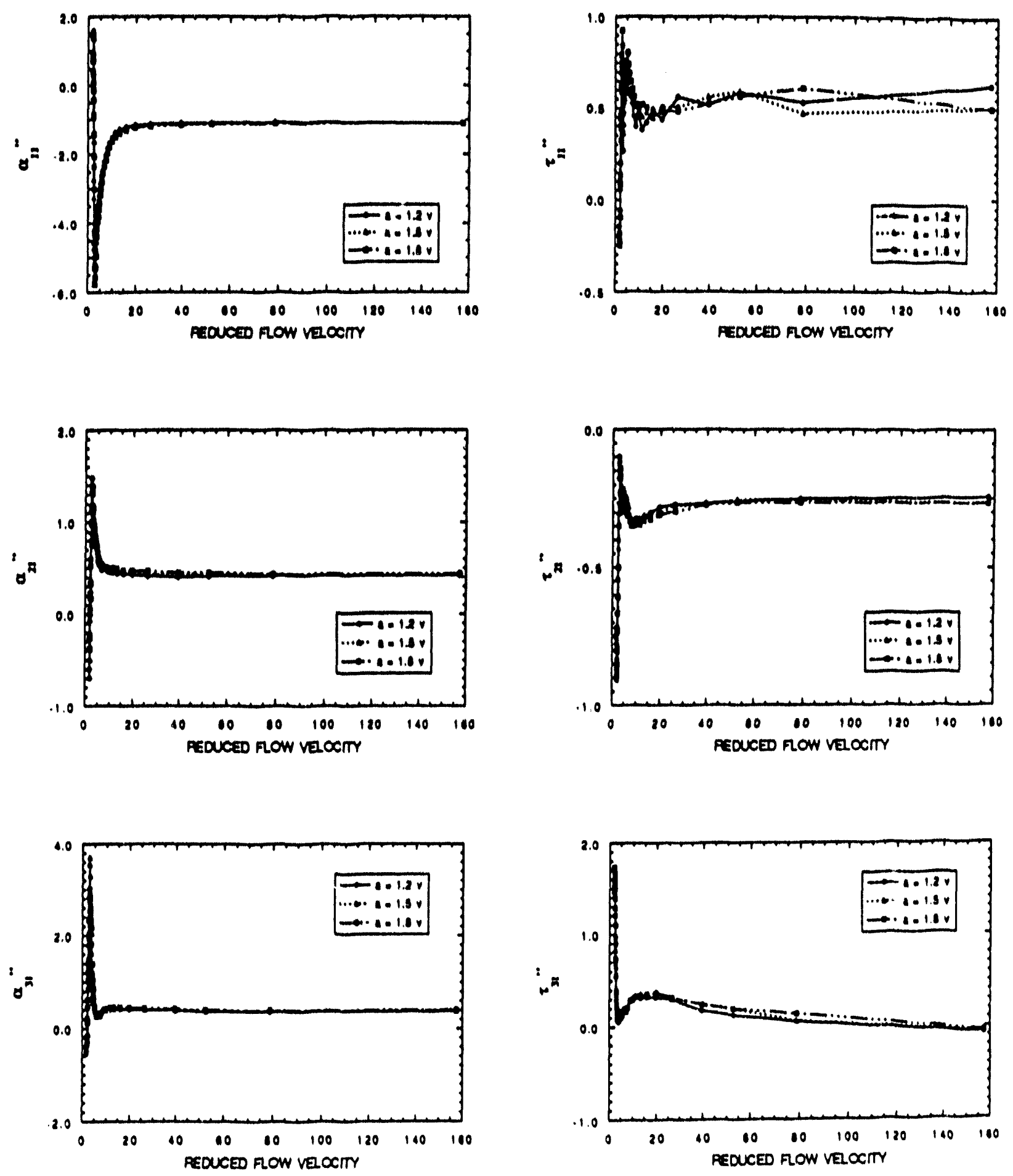

Fig. 21. Fluid-stiffness coefficients $\alpha_{11}^{\prime \prime}, \alpha_{21}^{\prime \prime}, \alpha_{31}^{\prime \prime}, \tau_{11}^{\prime \prime}, \tau_{21}^{\prime \prime}$, and $\tau_{31}^{\prime \prime}$, force transducer Type $B$, reduced flow velocity from 2 to 160 

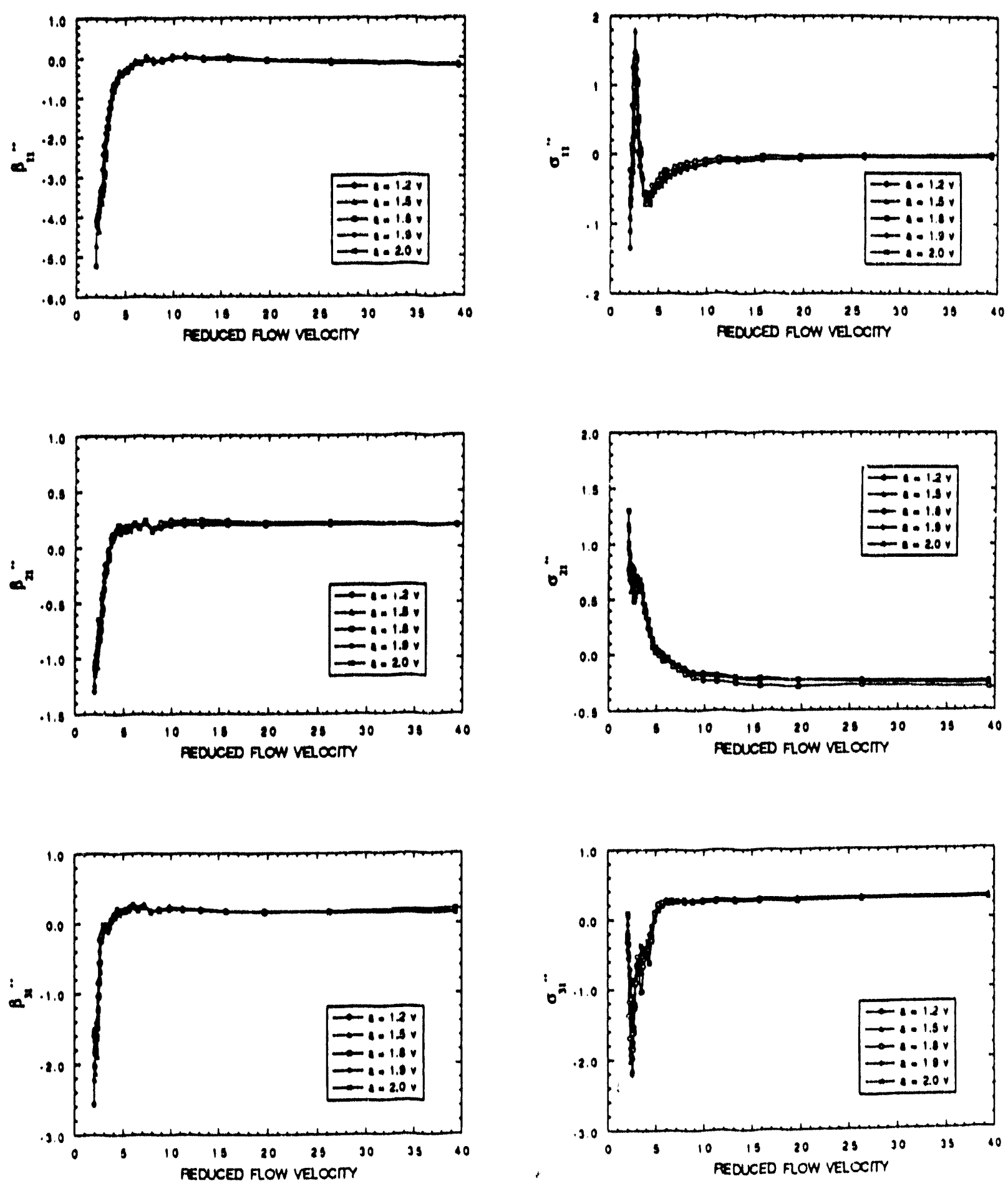

Fig. 22. Fluid-stiffiness coefficients $\beta_{11}^{\prime \prime}, \beta_{21}^{\prime \prime}, \beta_{31}^{\prime \prime}, \sigma_{11}^{\prime \prime}, \sigma_{21}^{\prime \prime}$, and $\sigma_{31}^{\prime \prime}$, force transducer Type B, reduced flou velocity from 2 to 40 

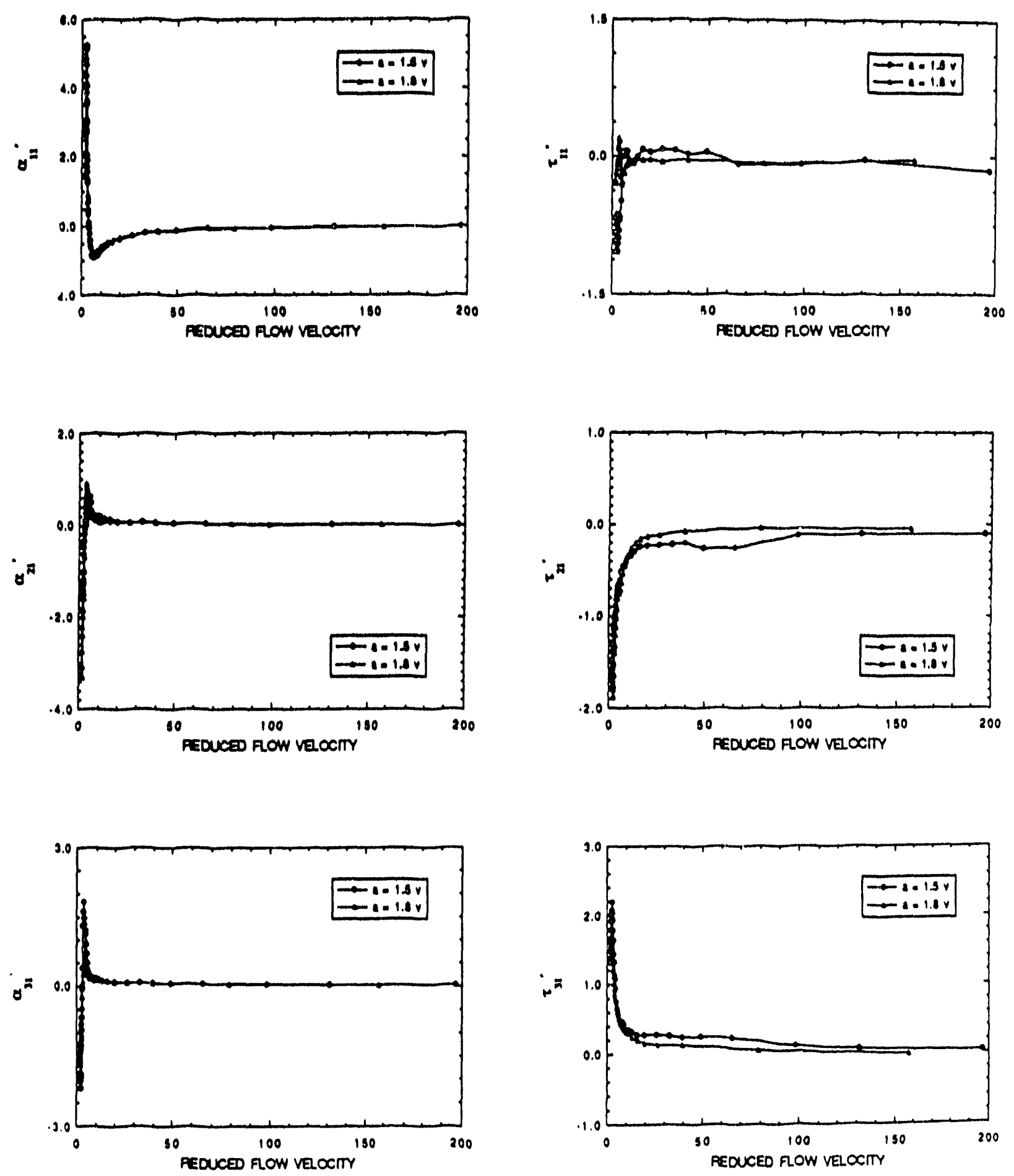

Fig. 23. Fluid-damping coefficients $\alpha_{11}^{\prime}, \alpha_{21}^{\prime}, \alpha_{31}^{\prime}, \tau_{11}^{\prime}, \tau_{21}^{\prime}$, and $\tau_{31}^{\prime}$, force transducer Type B, reduced flow velocity from 2 to 200 

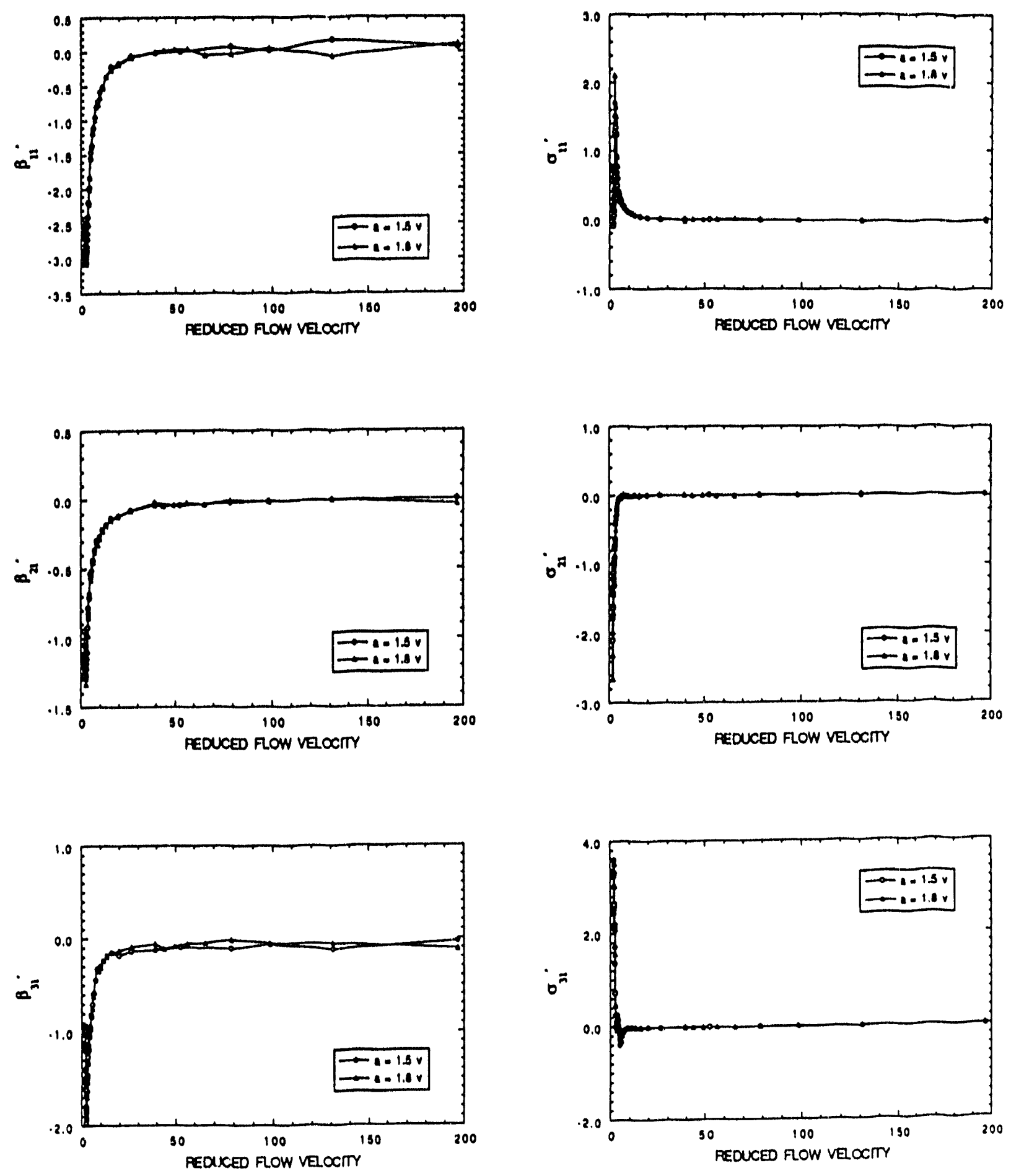

Fig. 24. Fluid-damping coefficients $\beta_{11}^{\prime}, \beta_{21}^{\prime}, \beta_{31}^{\prime}, \sigma_{11}^{\prime}, \sigma_{21}^{\prime}$, and $\sigma_{31}^{\prime}$, force transducer Type $B$, reduced flow velocity from 2 to 200 

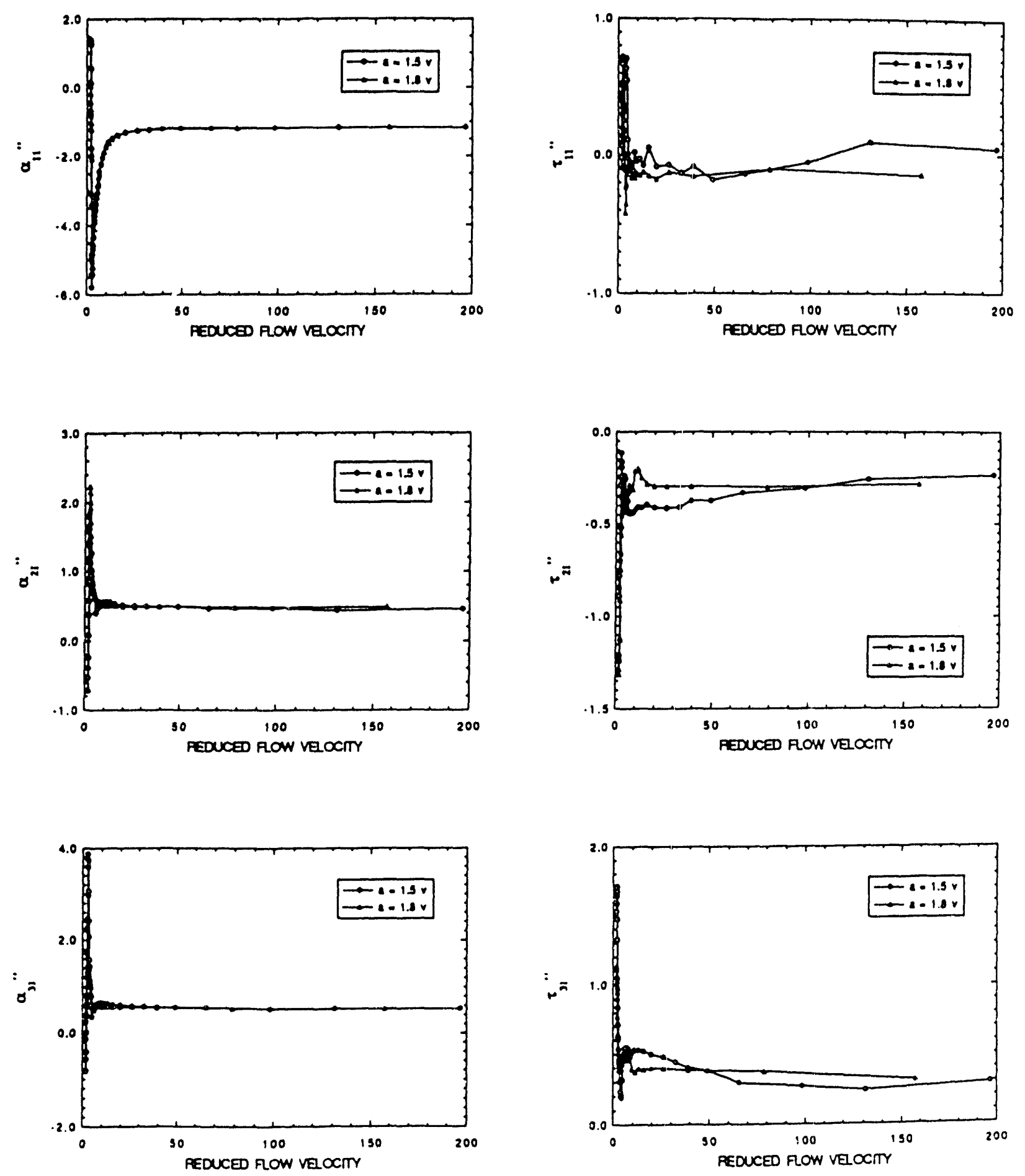

Fig. 25. Fluid-stiffness coefficients $\alpha_{11}^{\prime \prime}, \alpha_{21}^{\prime \prime}, \alpha_{31}^{\prime \prime}, \tau_{11}^{\prime \prime}, \tau_{21}^{\prime \prime}$, and $\tau_{31}^{\prime \prime}$, force transducer Type $B$, reduced flow velocity from 2 to 200 

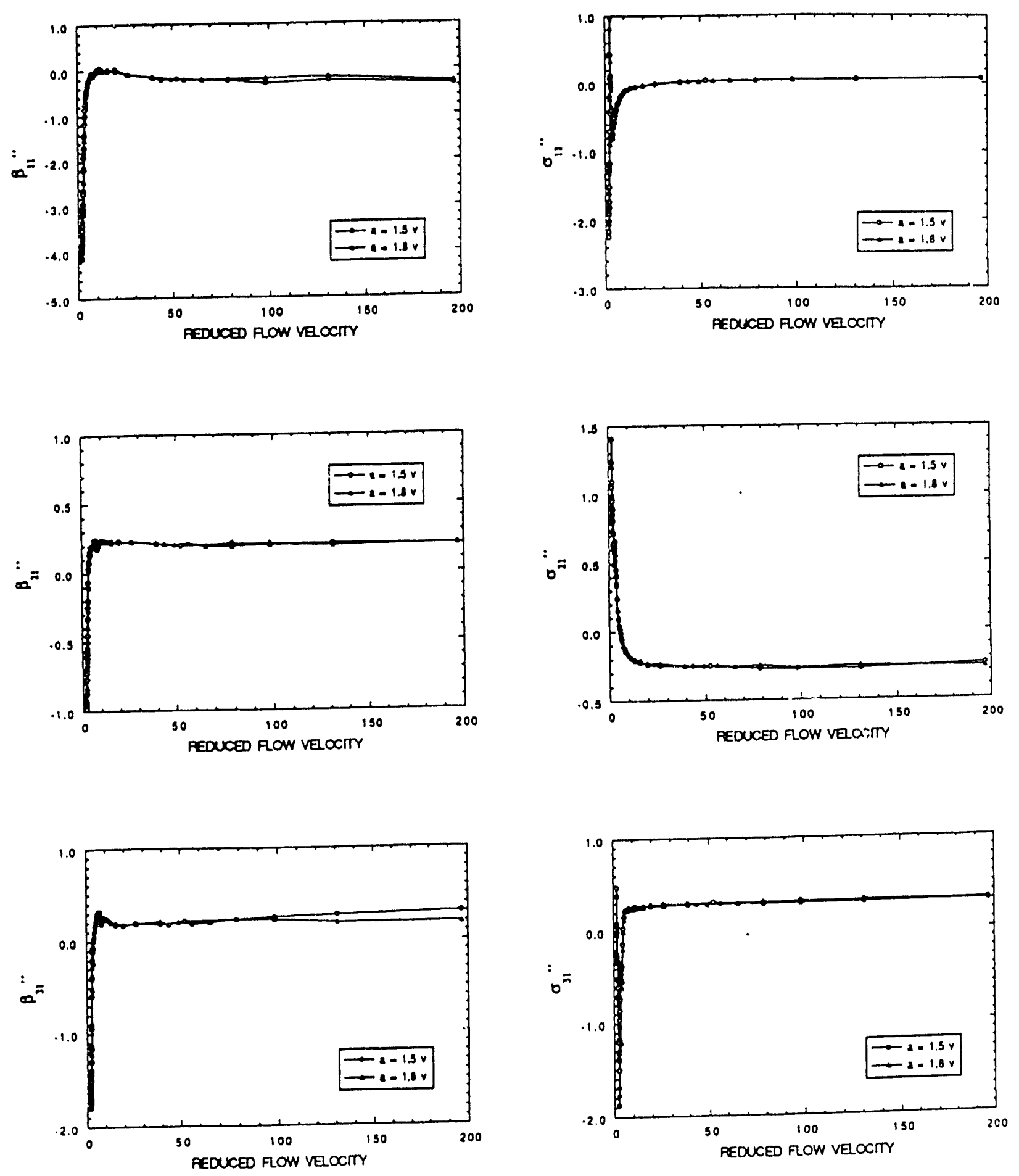

Fig. 26. Fluid-stiffness coefficients $\beta_{11}^{\prime \prime}, \beta_{21}^{\prime \prime}, \beta_{31}^{\prime \prime}, \sigma_{11}^{\prime \prime}, \sigma_{21}^{\prime \prime}$, and $\sigma_{31}^{\prime \prime}$, force transducer Type $B$, reduced flow velocity from 2 to 200 
Fluid-force coefficients obtained in this study agree reasonably well with those based on Tanaka's data (Tanaka 1980). However, some of the details are not in complete agreement. This is probably due to different experimental setup. The experimental parameters in the two tests were not identical. The flow across a tube row is fairly complicated, as demonstrated in the measurement of vortex shedding. Different flow patterns will affect the motion-dependent fluid forces. For example, the direction of $f_{2}$ due to the motion of $u_{1}$ is different from and opposite to the one measured by Tanaka and his colleagues $(1980,1981,1982)$. However, the direction of $f_{3}$ in this test is the same as $f_{2}$ in the test by Tanaka and his colleagues (1980), 1981,1982 ). It is apparent that the flow patterns in the two tests might he exactly opposite to each other.

\subsection{Effects of Oscillation Amplitudes and Excitation Frequencies}

To obtain reliable fluid forces, the excitation amplitudes of Tube 1 must vary with flow velocity and excitation frequency. Several series of measurements of fluid forces for various tube motions were performed to understand the effect of tube oscillation amplitude. Fluid force coefficients were obtained for various values of oscillation amplitude (d), which is the RMS displacement of Tube 1.

Figures 27-30 show the fluid force coefficients obtained with a Type-A force transducer for d varying from $1 \mathrm{~mm}$ to $4.5 \mathrm{~mm}$ at $0.12 \mathrm{~Hz}$ and from $1.2 \mathrm{~mm}$ to 3.7 $\mathrm{mm}$ at $1.2 \mathrm{~Hz}$. The flow velocity is $0.15 \mathrm{~m} / \mathrm{s}$; therefore, the corresponding reduced flow velocities are 50 and 5 for 0.12 and $1.2 \mathrm{~Hz}$ respectively.

Two flow velocities, $0.13 \mathrm{~m} / \mathrm{s}$ and $0.1 \mathrm{~m} / \mathrm{s}$, and two frequencies, 0.06 and 0.12 $\mathrm{Hz}$ were tested with a Type- $\mathrm{B}$ force transducer. The corresponding reduced flow velocities were 42.5 and 85 for $0.13 \mathrm{~m} / \mathrm{s}$ at 0.12 and $0.06 \mathrm{~Hz}$ and 32.8 and 65.5 for $0.1 \mathrm{~m} / \mathrm{s}$ at 0.12 and $0.06 \mathrm{~Hz}$. The results are given in Figs. 31-38.

From Figs. 27-38, it is evident that there is no noticeable difference. The maximum peak magnitude of $6.36 \mathrm{~mm}$ (RMS $4.5 \mathrm{~mm}$ ) is $\approx 70 \%$ of the gap between tubes. The amplitude of tube displacement has been tested at higher values than those of Tanaka and his colleagues (1980,1981, 1982); the results shown agree well with their data.

Although fluid-force coefficients are not affected by ais uscillation amplitude $\equiv 70 \%$ of the gap, larger oscillation amplitudes are expected to have a significant influence. As demonstrated in a stability study (Chen 1987a), two instability limits, intrinsic and excited, are encountered. The difference between these two instability limits is partially due to the dependence of fluid-force coefficients on oscillation amplitude. This can be noted in Figs. 19-22. At the largest displacement, all fluid- 

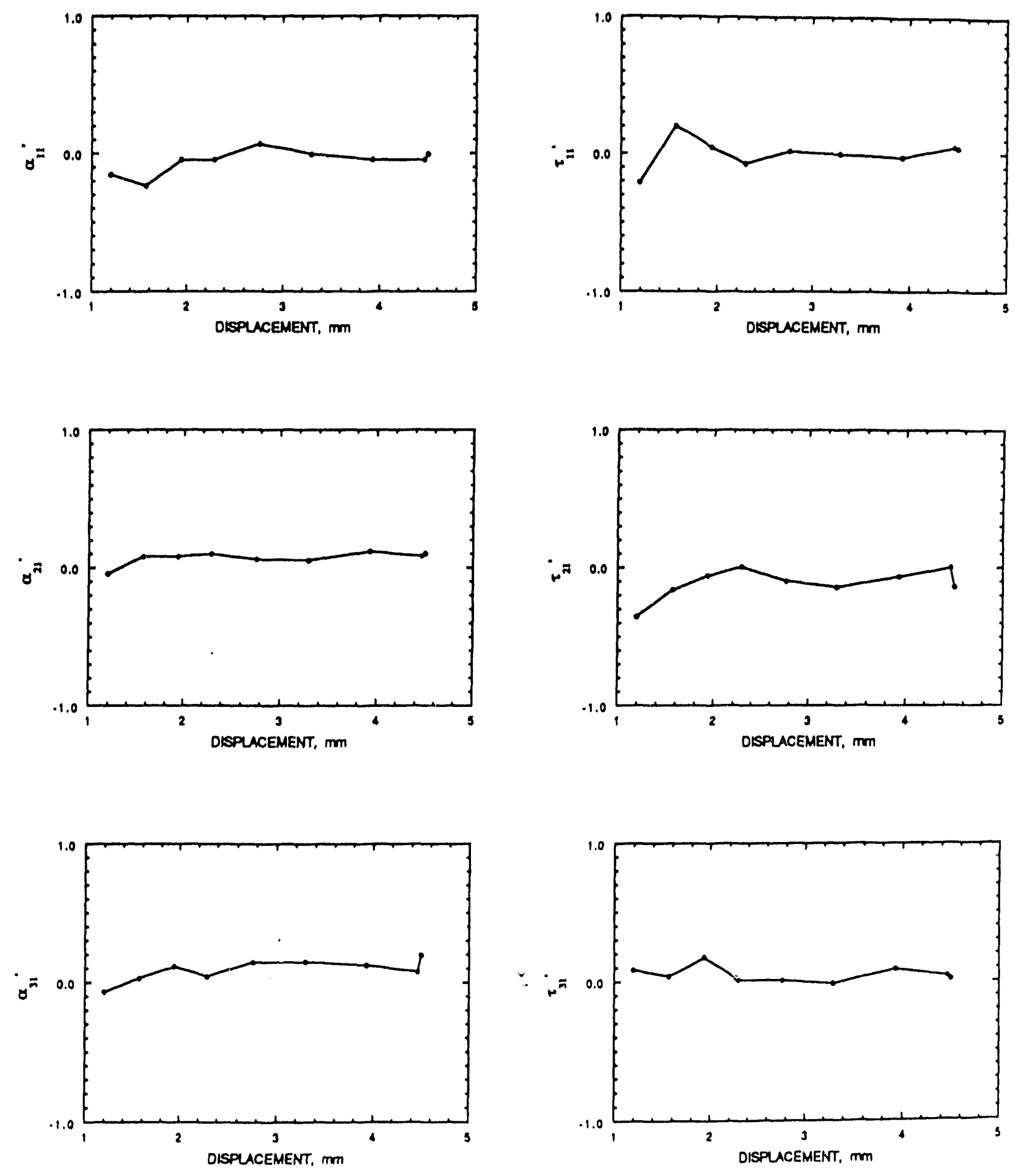

Fig. 27. Fluid-damping coefficients $\alpha_{11}^{\prime}, \alpha_{21}^{\prime}, \alpha_{31}^{\prime}, \tau_{11}^{\prime}, \tau_{21}^{\prime}$, and $\tau_{31}^{\prime}$ as a function of RMS tube displacement oscillating at $0.12 \mathrm{~Hz}$, force transducer Type A 

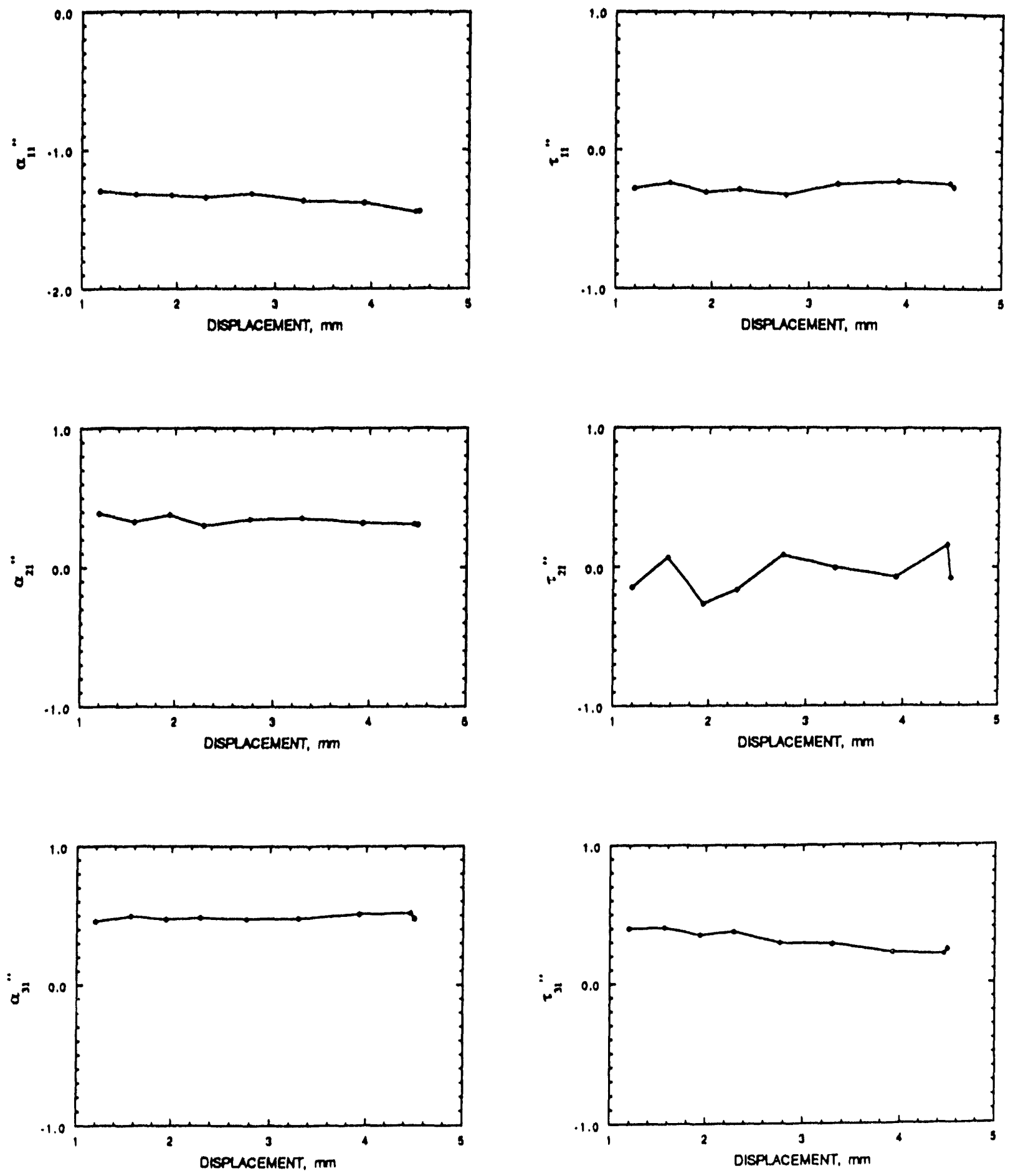

Fig. 28. Fluid-stiffness coefficients $\alpha_{11}^{\prime \prime}, \alpha_{21}^{\prime \prime}, \alpha_{31}^{\prime \prime}, \tau_{11}^{\prime \prime}, \tau_{21}^{\prime \prime}$, and $\tau_{31}^{\prime \prime}$ as a function of RMS tube displacement oscillating at $0.12 \mathrm{~Hz}$, force transducer Type $A$ 

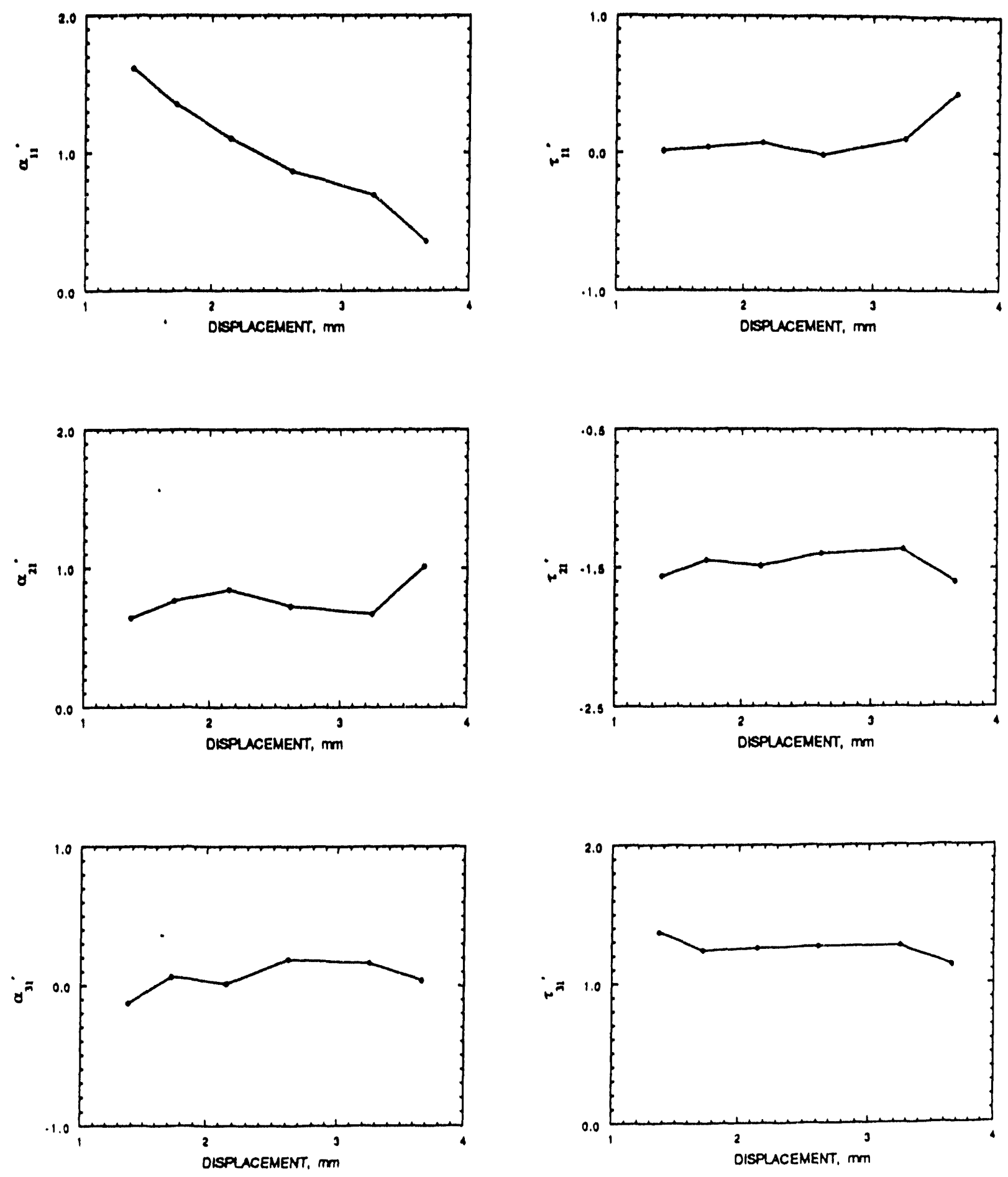

Fig. 29. Fluid-damping coefficients $\alpha_{11}^{\prime}, \alpha_{21}^{\prime}, \alpha_{31}^{\prime}, \tau_{11}^{\prime}, \tau_{21}^{\prime}$, and $\tau_{31}^{\prime}$ as a function of RMS tube displacement oscillating at $1.2 \mathrm{~Hz}$, force transducer Type A 

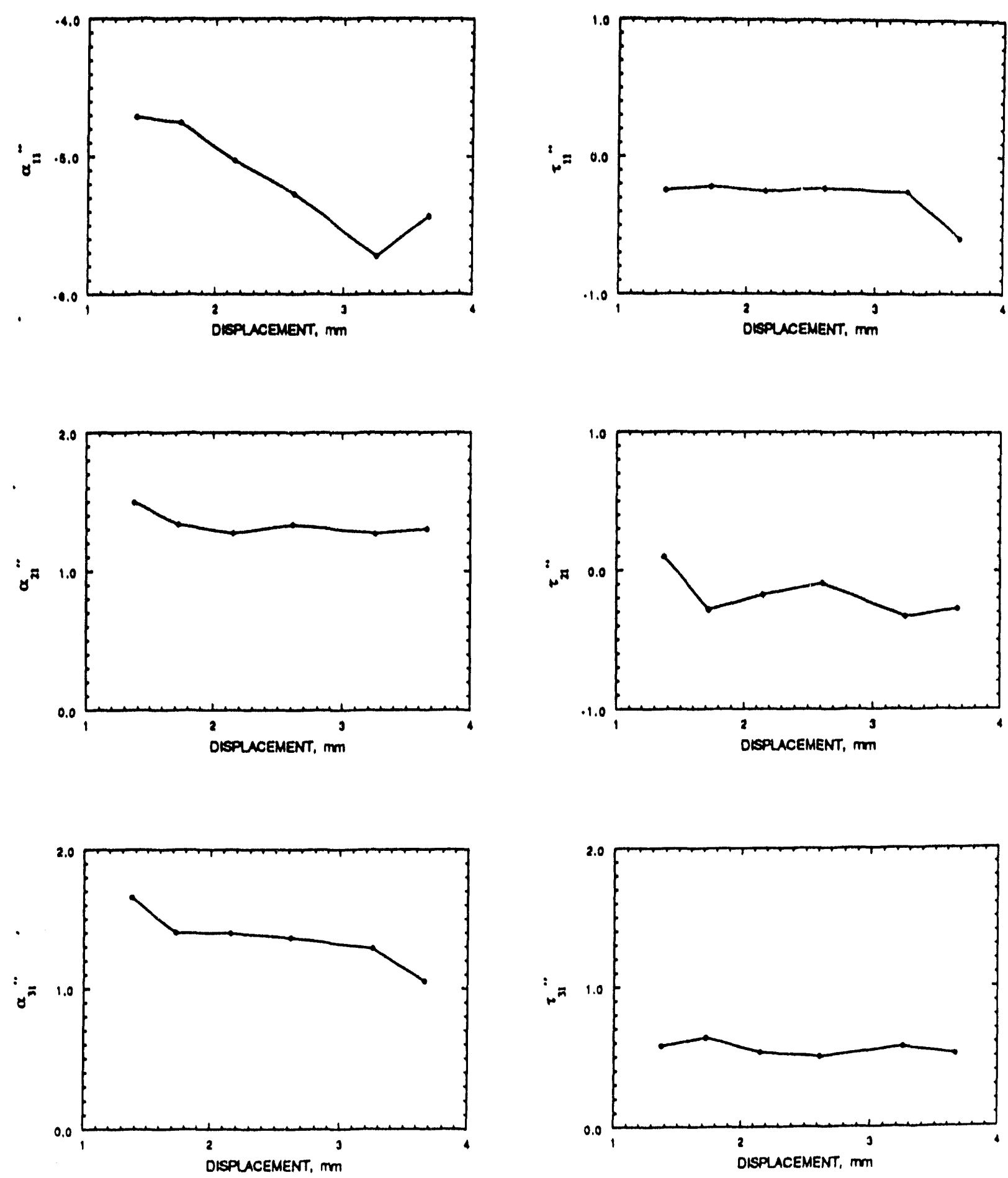

Fig. 30. Fluid-stiffness coefficients $\alpha_{11}^{\prime \prime}, \alpha_{21}^{\prime \prime}, \alpha_{31}^{\prime \prime}, \tau_{11}^{\prime \prime}, \tau_{21}^{\prime \prime}$, and $\tau_{31}^{\prime \prime}$ as a function of RMS tube displacement oscillating at $1.2 \mathrm{~Hz}$, force transducer Type $\mathrm{A}$ 

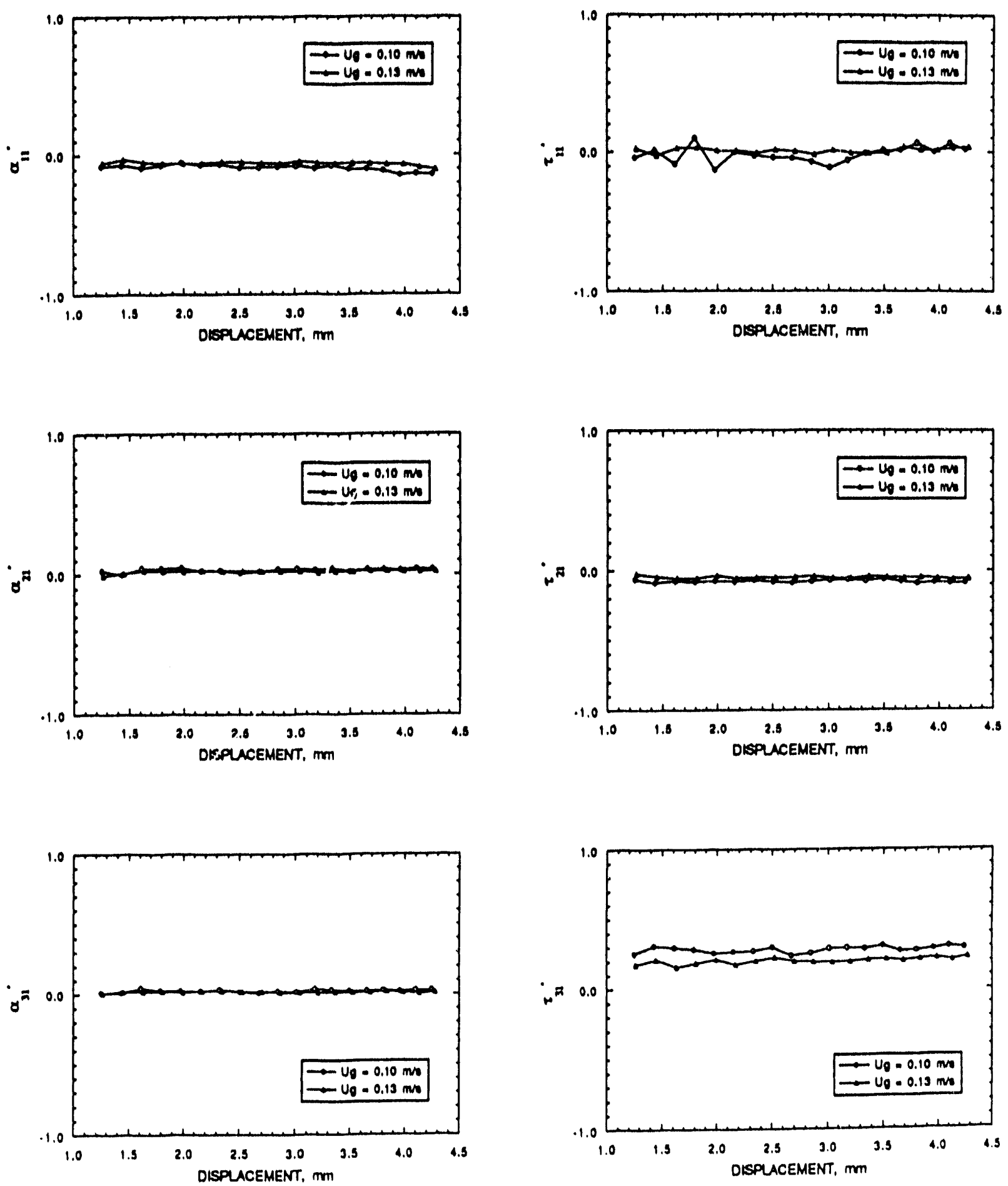

Fig. 31. Fluid-damping coefficients $\alpha_{11}^{\prime}, \alpha_{21}^{\prime}, \alpha_{31}^{\prime}, \tau_{11}^{\prime}, \tau_{21}^{\prime}$, and $\tau_{31}^{\prime}$ as a function of RMS tube displacement oscillating at $0.06 \mathrm{~Hz}$, force transducer Type B 

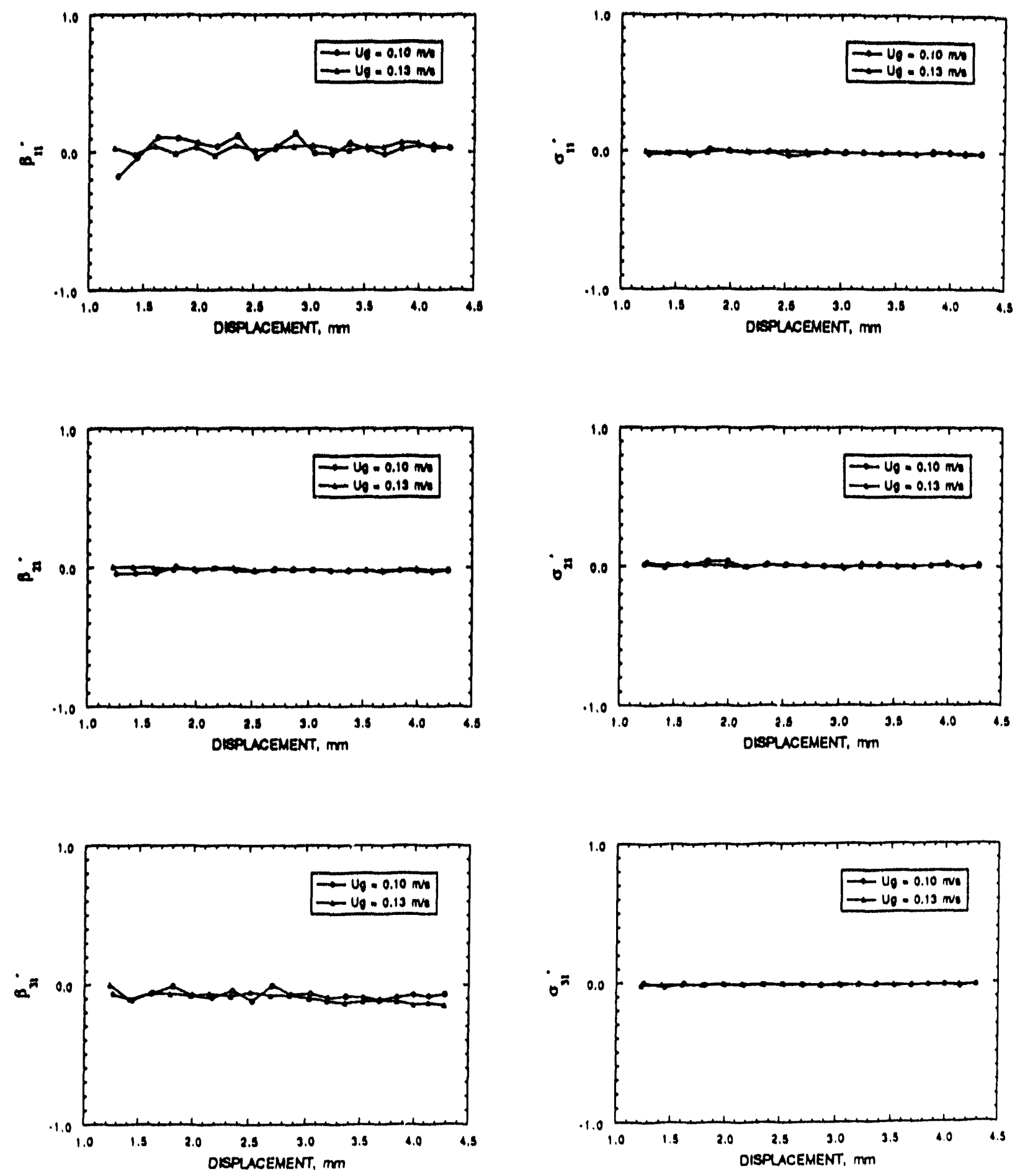

Fig. 32. Fluid-damping coefficients $\beta_{11}^{\prime}, \beta_{21}^{\prime}, \beta_{31}^{\prime}, \sigma_{11}^{\prime}, \sigma_{21}^{\prime}$, and $\sigma_{31}^{\prime}$ as a function of RMS tube displacement oscillating at $0.06 \mathrm{~Hz}$, force transducer Type $B$ 

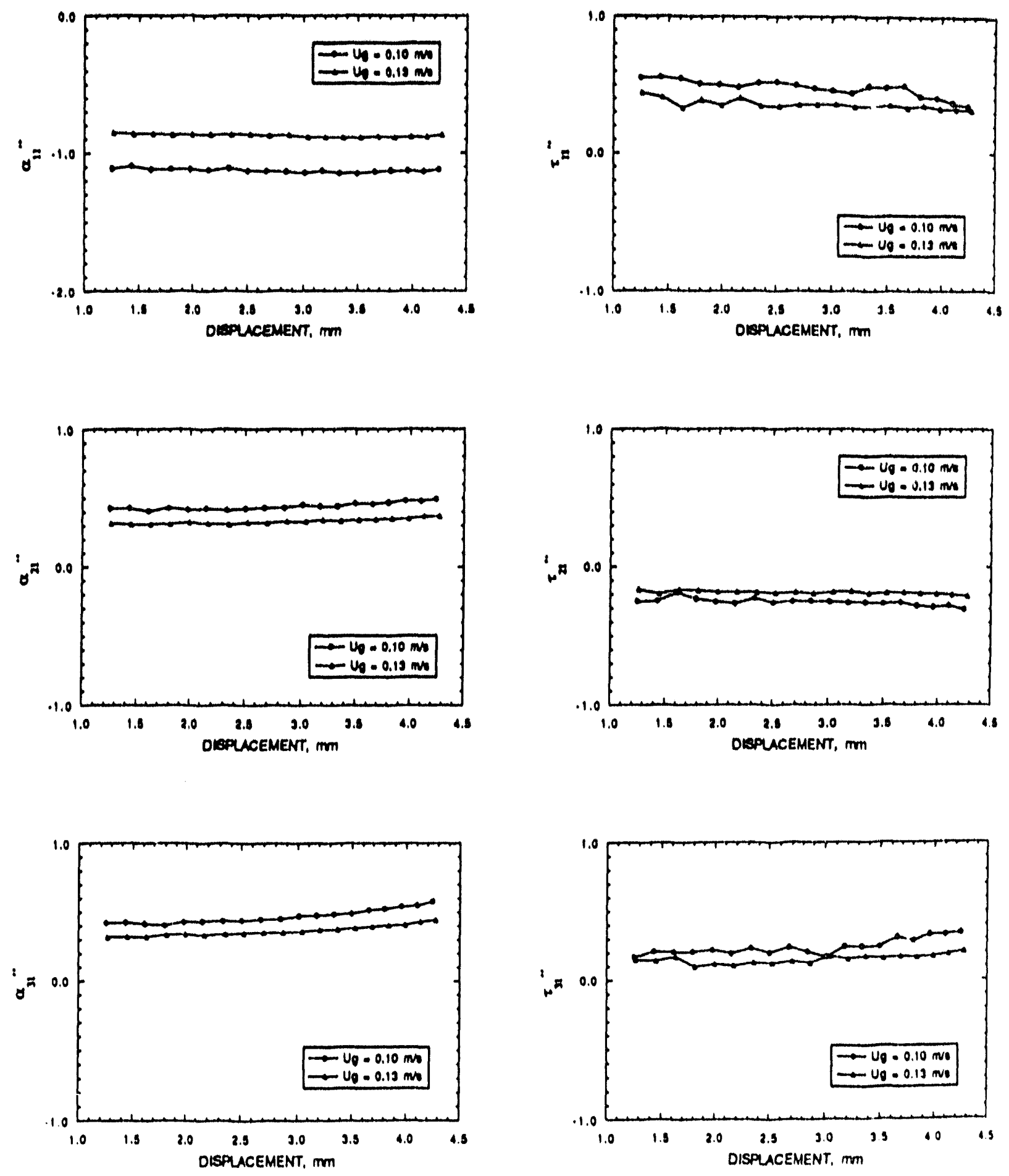

Fig. 33. Fluid-stiffness coefficients $\alpha_{11}^{\prime \prime}, \alpha_{21}^{\prime \prime}, \alpha_{31}^{\prime \prime}, \tau_{11}^{\prime \prime}, \tau_{21}^{\prime \prime}$, and $\tau_{31}^{\prime \prime}$ as a function of RMS tube displacement oscillating at $0.06 \mathrm{~Hz}$, force transducer "Type $B$ 

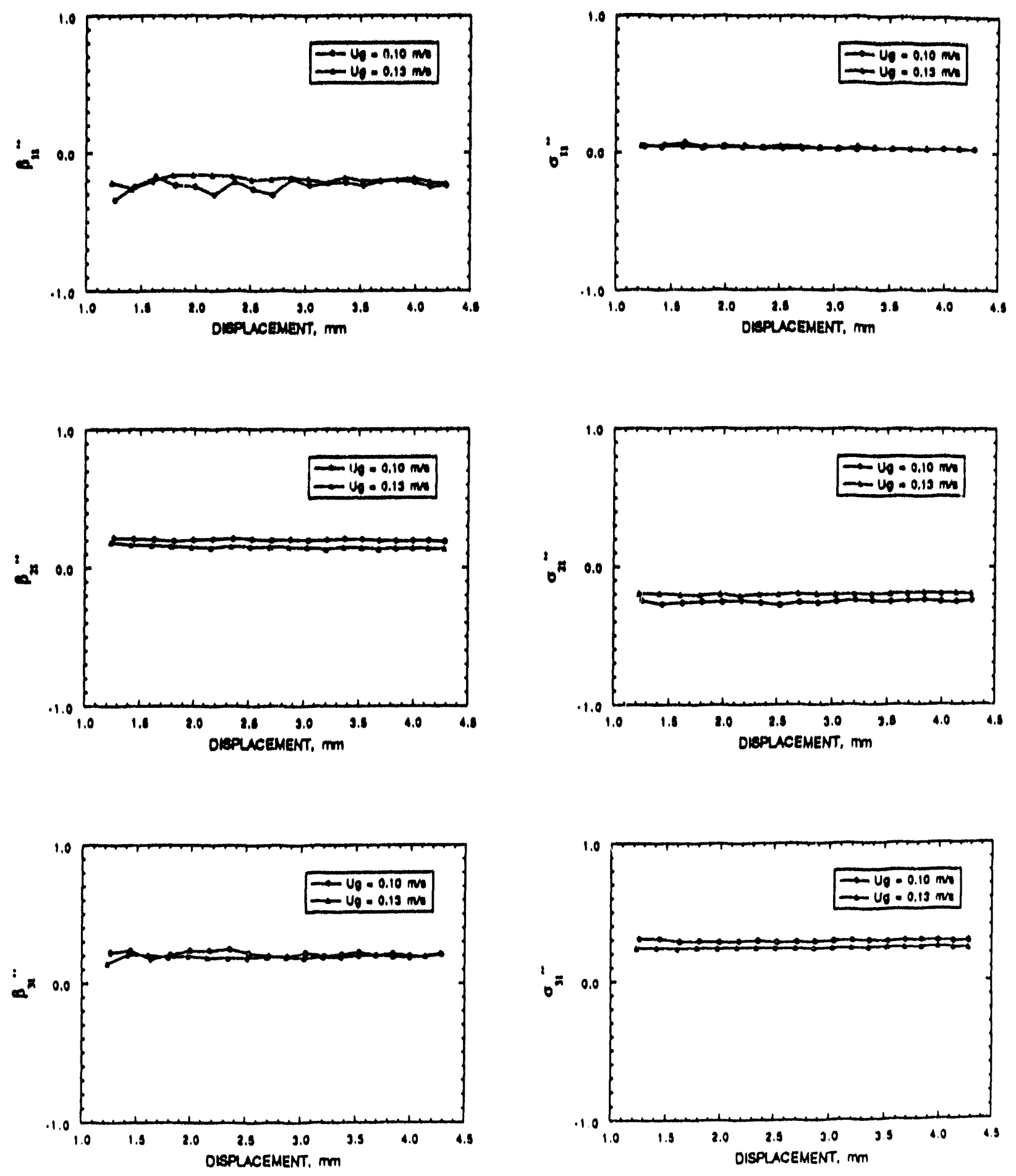

Fig. 34. Fluid-stiffness coefficients $\beta_{11}^{\prime \prime}, \beta_{21}^{\prime \prime}, \beta_{31}^{\prime \prime}, \sigma_{11}^{\prime \prime}, \sigma_{21}^{\prime \prime}$, and $\sigma_{31}^{\prime \prime}$ as a function of RMS tube displacement oscillating at $0.06 \mathrm{~Hz}$, force transducer Type $B$ 

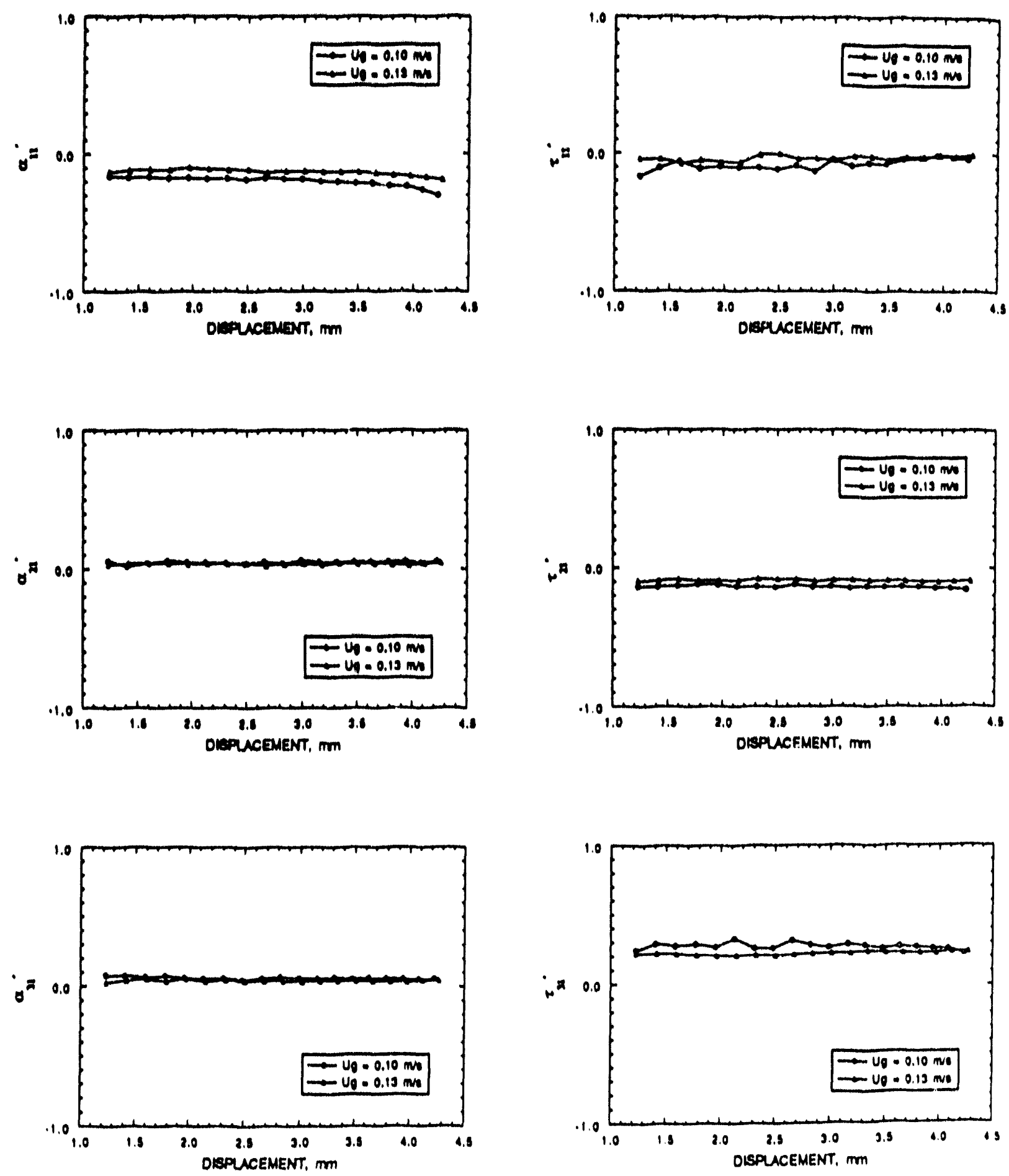

Fig. 35. Fluid-damping coefficients $\alpha_{11}^{\prime}, \alpha_{21}^{\prime}, \alpha_{31}^{\prime}, \tau_{11}^{\prime}, \tau_{21}^{\prime}$, and $\tau_{31}^{\prime}$ as a function of RMS tube displacement oscillating at $0.12 \mathrm{~Hz}$, force transducer Type $B$ 

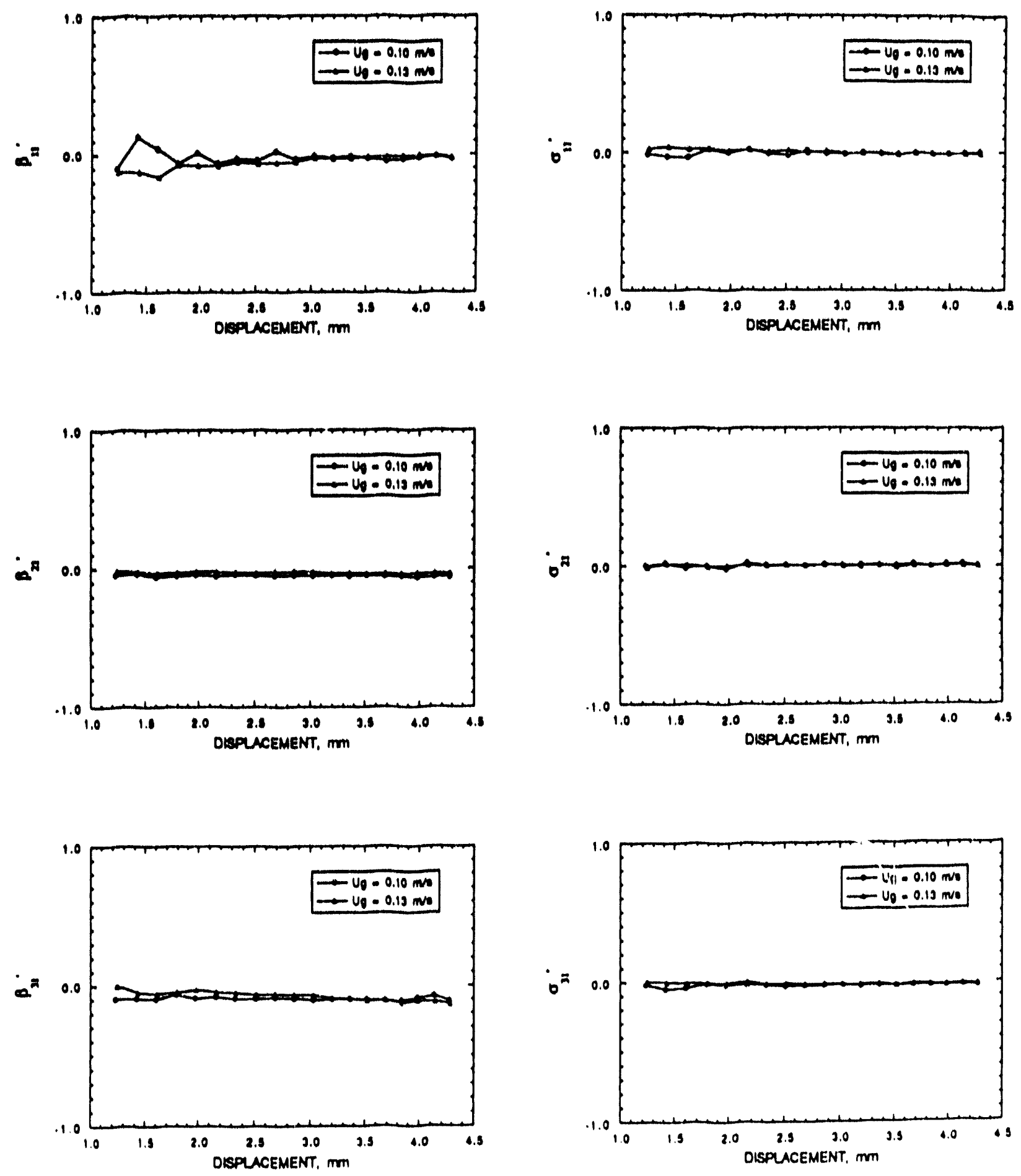

Fig. 36. Fluid-damping coefficients $\beta_{11}^{\prime}, \beta_{21}^{\prime}, \beta_{31}^{\prime}, \sigma_{11}^{\prime}, \sigma_{21}^{\prime}$, and $\sigma_{31}^{\prime}$ as a function of RMS tube displacement oscillating at $0.12 \mathrm{~Hz}$, force transducer Type $B$ 

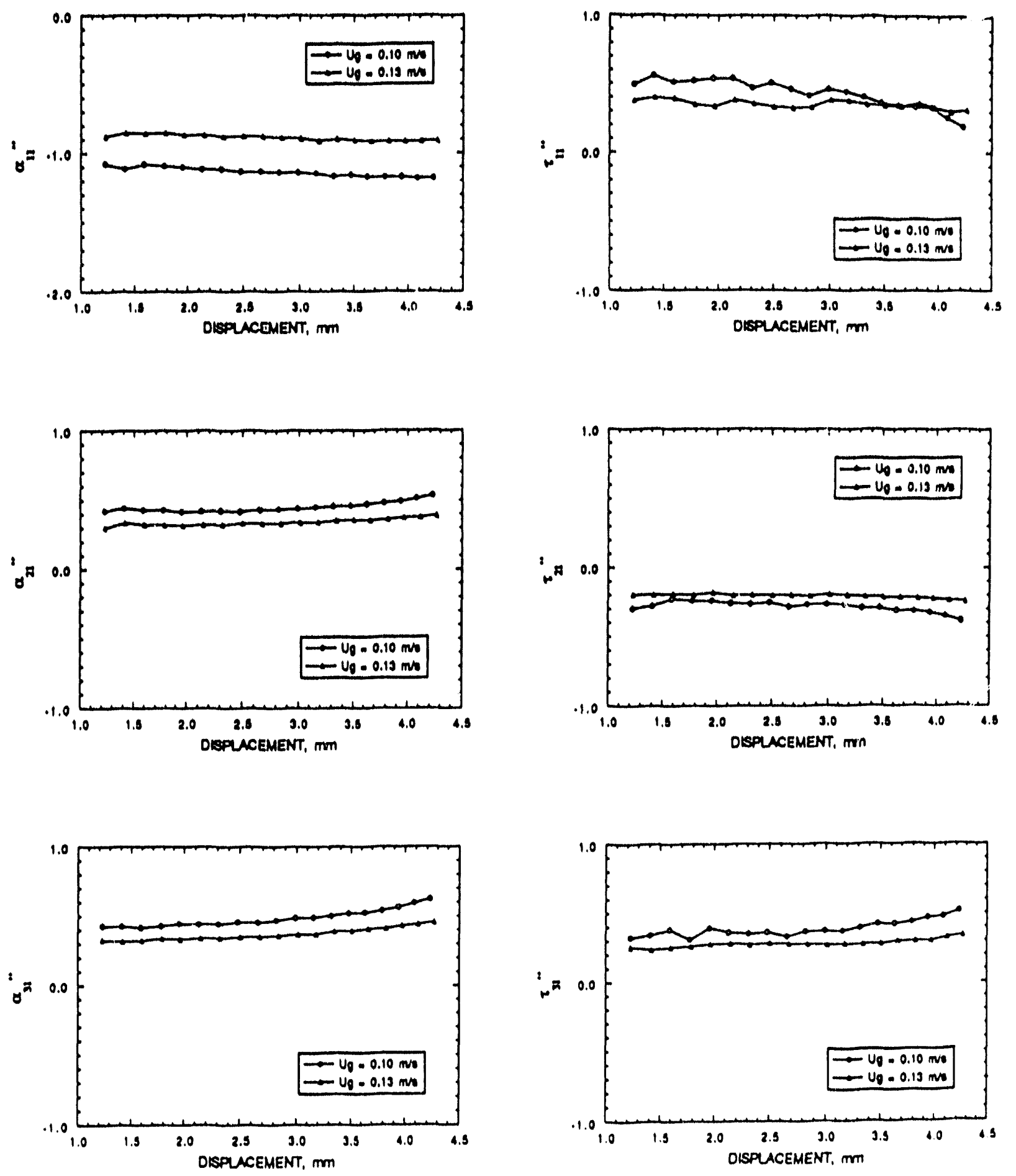

Fig. 37. Fluid-stiffness coefficients $\alpha_{11}^{\prime \prime}, \alpha_{21}^{\prime \prime}, \alpha_{31}^{\prime \prime}, \tau_{11}^{\prime \prime}, \tau_{21}^{\prime \prime}$, and $\tau_{31}^{\prime \prime}$ as a function of RMS tube displacement oscillating at $0.12 \mathrm{~Hz}$, force transducer Type B 

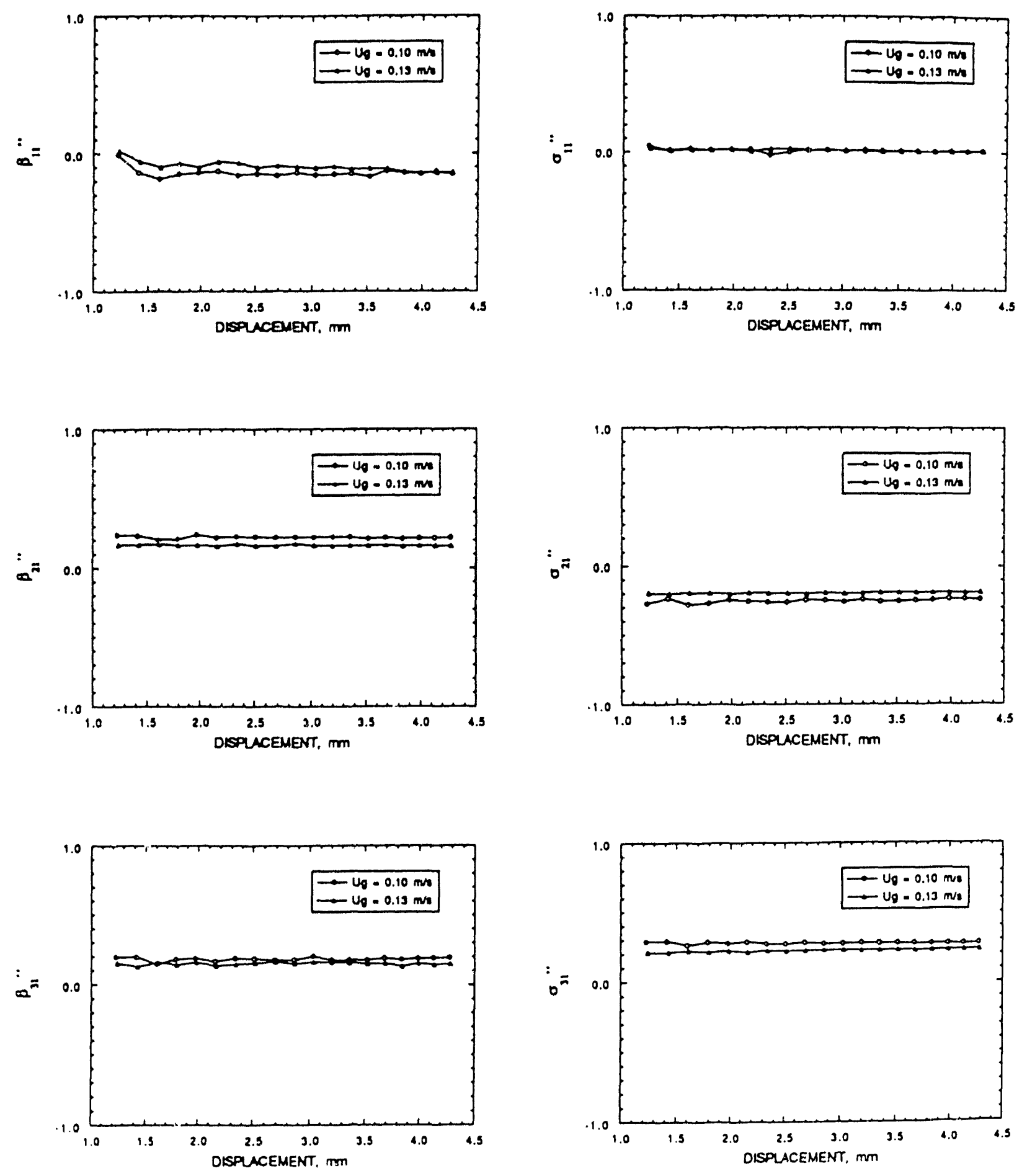

Fig. 38. Fluid-stiffness coefficients $\beta_{11}^{\prime \prime}, \beta_{21}^{\prime \prime}, \beta_{31}^{\prime \prime}, \sigma_{11}^{\prime \prime}, \sigma_{21}^{\prime \prime}$, and $\sigma_{31}^{\prime \prime}$ as a function of RMS tube displacement oscillating at $0.12 \mathrm{~Hz}$, force transducer Type $B$ 
force coefficients begin to change significantly. To protect the integrity of the force transducers, larger amplitude oscillations were not tested.

The effect of tube oscillation amplitude can be summarized as follows. Because fluid-force coefficients are independent of tube oscillation amplitude if the tube displacement is less than $=70 \%$ of the gap, small-amplitude oscillations of the tubes due to other excitation sources are not expected to affect the threshold of fluidelastic instability. However, if the tubes are excited to perform large-amplitude oscillations, the nonlinear characteristics of fluid forces become important. In predicting tube motion in the post critical region, nonlinear fluid-force coefficients will be needed to predict nonlinear behavior, including chaotic motion, more accurately.

\subsection{Symmetry and Antisymmetry of Fluid Forces}

The symmetric or antisymmetric characteristics of the fluid-force coefficients provide some insights into their effects on fluidelastic instability of tube arrays in crossflow. The details are given by Chen (1987a) in the form of a generalized theory for fluidelastic instability of a fluid-structure system. The symmetric or antisymmetric properties of added mass, fluid damping, and fluid stiffness for fluidelastic instability of tube arrays considered here are based on the data obtained in this test.

The added mass coefficients are symmetric and have been proved by the potential flow theory for small-amplitude oscillations (Chen 1987a). In a viscous fluid, it is believed that the added mass coefficients are also symmetric for smallamplitude oscillations. Although this has not been proved rigorously, numerical results and experimental data are in agreement with this observation. Based on the potential flow theory, the added mass matrix for a row of three active tubes with $\mathrm{T} / \mathrm{D}=1.35$ can be written as

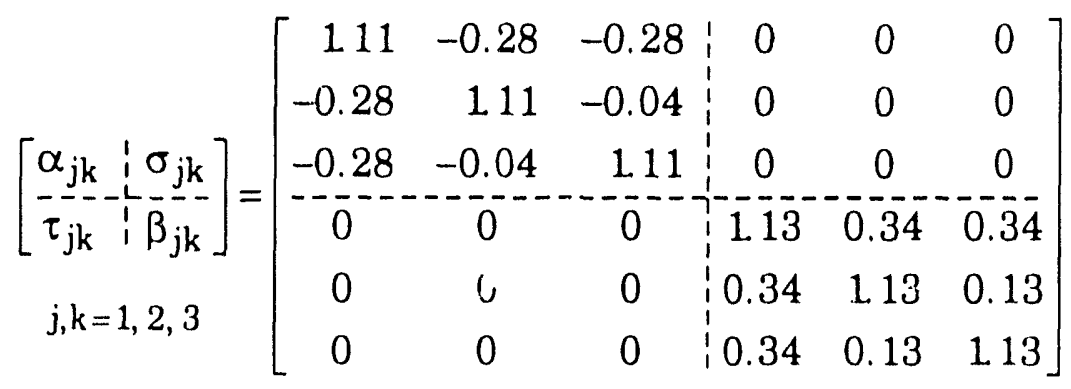

It is apparent that the added mass matrix is symmetric. 
For fluid-damping and fluid-stiffness coefficients, no such symmetric property exists. In the past, the use of symmetric and antisymmetric properties has been based on physical and geometrical considerations. The coefficient matrices for a tube row are assumed to be of the following form:

Matrix for fluid-damping coefficient:

$$
\left[\begin{array}{c:c}
\alpha_{j \mathrm{k}}^{\prime} & \sigma_{j \mathrm{kk}}^{\prime} \\
\hdashline \tau_{j \mathrm{k}} & \beta_{j \mathrm{k}}
\end{array}\right]=\left[\begin{array}{ccc:ccc}
\alpha_{11}^{\prime} & \alpha_{12}^{\prime} & \alpha_{12}^{\prime} & 0 & \sigma_{12}^{\prime} & -\sigma_{12}^{\prime} \\
\alpha_{12}^{\prime} & \alpha_{11}^{\prime} & 0 & -\sigma_{12}^{\prime} & 0 & 0 \\
\alpha_{12}^{\prime} & 0 & \alpha_{11}^{\prime} & \sigma_{12}^{\prime} & 0 & 0 \\
\hdashline 0 & \tau_{12}^{\prime} & -\tau_{12}^{\prime} & \beta_{11}^{\prime} & \beta_{12}^{\prime} & \beta_{12}^{\prime} \\
-\tau_{12}^{\prime} & 0 & 0 & \beta_{12}^{\prime} & \beta_{11}^{\prime} & 0 \\
\tau_{12}^{\prime} & 0 & 0 & \beta_{12}^{\prime} & 0 & \beta_{11}^{\prime}
\end{array}\right]
$$

Matrix for fluid-stiffness coefficient:

$$
\left[\begin{array}{c:c}
\alpha_{j k}^{\prime \prime} & \sigma_{j \mathrm{k}}^{\prime \prime} \\
\hdashline \tau_{\mathrm{jk}}^{\prime \prime} & \beta_{\mathrm{jk}}^{\prime \prime}
\end{array}\right]=\left[\begin{array}{ccc:ccc}
\alpha_{11}^{\prime \prime} & \alpha_{12}^{\prime \prime} & \alpha_{12}^{\prime \prime} & 0 & \sigma_{12}^{\prime \prime} & -\sigma_{12}^{\prime \prime} \\
\alpha_{12}^{\prime \prime} & \alpha_{11}^{\prime \prime} & 0 & -\sigma_{12}^{\prime \prime} & 0 & 0 \\
\alpha_{12}^{\prime \prime} & 0 & \alpha_{11}^{\prime \prime} & \sigma_{12}^{\prime \prime} & 0 & 0 \\
\hdashline 0 & \tau_{12}^{\prime \prime} & -\tau_{12}^{\prime \prime} & \beta_{11}^{\prime \prime} & \beta_{12}^{\prime \prime} & \beta_{12}^{\prime \prime} \\
-\tau_{12}^{\prime \prime} & 0 & 0 & \beta_{12}^{\prime \prime} & \beta_{11}^{\prime \prime} & 0 \\
\tau_{12}^{\prime \prime} & 0 & 0 & \beta_{12}^{\prime \prime} & 0 & \beta_{11}^{\prime \prime}
\end{array}\right]
$$

Test results from this experiment can be used to verify the validity of these assumptions.

In general, Figs. 15-26, which show fluid force coefficients $\alpha_{21}^{\prime}, \alpha_{31}^{\prime}, \sigma_{21}^{\prime}, \sigma_{31}^{\prime}, \tau_{21}^{\prime}$, $\tau_{31}^{\prime}, \beta_{21}^{\prime}, \beta_{31}^{\prime}, \alpha_{21}^{\prime \prime}, \alpha_{31}^{\prime \prime}, \sigma_{21}^{\prime \prime}, \sigma_{31}^{\prime \prime}, \tau_{21}^{\prime \prime}, \tau_{31}^{\prime \prime}, \beta_{21}^{\prime \prime}$, and $\beta_{31}^{\prime \prime}$, indicate that

$$
\begin{aligned}
& \alpha_{21}^{\prime}=\alpha_{31}^{\prime}, \\
& \alpha_{21}^{\prime}=-\sigma_{31}^{\prime}, \\
& \tau_{21}^{\prime}=-\tau_{31}^{\prime}, \\
& \beta_{21}^{\prime}=\beta_{31}^{\prime},
\end{aligned}
$$

and 


$$
\begin{aligned}
& \alpha_{21}^{\prime \prime}=\alpha_{31}^{\prime \prime}, \\
& \alpha_{21}^{\prime \prime}=-\sigma_{31}^{\prime \prime}, \\
& \tau_{21}^{\prime \prime}=-\tau_{31}^{\prime \prime}, \\
& \beta_{21}^{\prime \prime}=\beta_{31}^{\prime \prime} .
\end{aligned}
$$

These equations agree with Eqs. 13 and 14. As pointed out earlier, there is a minor difference between our test and that of Tanaka and his colleagues (1980, 1981, 1982 ), in which the signs of $\tau_{21}^{\prime \prime}$ are opposite at high reduced flow velocities. This difference is believed to be associated with the different patterns used by us and them.

\section{Unsteady Flow Theory for Fluidelastic Instability of Tube Arrays}

\subsection{Equations of Motion}

Consider a group of $n$ identical tubes with radius $R(=D / 2)$ subjected to crossflow as shown in Fig. 1. The variables associated with the tube motion in the $x$ and $y$ directions are flexural rigidity EI, tube mass per unit length $\mathrm{m}$, structural damping coefficient $\mathrm{C}_{s}$, and displacements $\mathrm{u}_{\mathrm{j}}$ and $\mathrm{v}_{\mathrm{j}}$. The equations of motion for tube $\mathrm{j}$ in the $\mathrm{x}$ and $\mathrm{y}$ directions are (Chen $1987 \mathrm{~b}$ )

$$
\begin{aligned}
& E I \frac{\partial^{4} u_{j}}{\partial z^{4}}+ C_{s} \frac{\partial u_{j}}{\partial t}+m \frac{\partial^{2} u_{j}}{\partial t^{2}}+\sum_{k=1}^{n} \rho \pi R^{2}\left(\alpha_{j k} \frac{\partial^{2} u_{k}}{\partial t^{2}}+\sigma_{j k} \frac{\partial^{2} v_{k}}{\partial t^{2}}\right) \\
&-\sum_{k=1}^{n}\left(\frac{\rho U^{2}}{\omega} \alpha_{j k}^{\prime} \frac{\partial u_{k}}{\partial t}+\frac{\rho U^{2}}{\omega} \sigma_{j k}^{\prime} \frac{\partial v_{k}}{\partial t}\right)-\sum_{k=1}^{n} \rho U^{2}\left(\alpha_{j k}^{\prime \prime} u_{k}+\sigma_{j k}^{\prime \prime} v_{k}\right)=0 \\
& \operatorname{EII} \frac{\partial^{4} v_{j}}{\partial z^{4}}+C_{s} \frac{\partial v_{j}}{\partial t}+m \frac{\partial^{2} v_{i}}{\partial t^{2}}+\sum_{k=1}^{n} \rho \pi R^{2}\left(\tau_{j k} \frac{\partial^{2} u_{k}}{\partial t^{2}}+\beta_{j k} \frac{\partial^{2} v_{k}}{\partial t^{2}}\right) \\
&-\sum_{k=1}^{n}\left(\frac{\rho U^{2}}{\omega} \tau_{j k}^{\prime} \frac{\partial u_{k}}{\partial t}+\frac{\rho U^{2}}{\omega} \beta_{j k}^{\prime} \frac{\partial v_{k}}{\partial t}\right)-\sum_{k=1}^{n} \rho U^{2}\left(\tau_{j k}^{\prime \prime} u_{k}+\beta_{j k}^{\prime \prime}\right)=0
\end{aligned}
$$


Note that fluid-damping coefficients and fluid-stiffness coefficients are functions of reduced flow velocity $U_{r}\left(=U / f_{f} D ; f_{f}\right.$ is the oscillation frequency of the tubes in flow).

The in-vacuum variables are mass per unit length $\mathrm{m}$, modal damping ratio $\zeta_{\mathrm{v}}$, and natural frequency $f_{v}\left(=\omega_{v} / 2 \pi\right)$. The values for $f_{v}$ and $\zeta_{v}$ can be calculated from the equation of motion and appropriate boundary conditions or from tests in vacuum (practically in air). The modal function of a tube vibrating in vacuum and in fluid is $\psi(z)$;

$$
\frac{1}{\ell} \int_{0}^{1} \psi^{2}(\mathrm{z}) \mathrm{dz}=1
$$

where $\ell$ is the length of the tubes. Let

$$
u_{j}(z, t)=a_{j}(t) \psi(z)
$$

and

$$
v_{j}(z, t)=b_{j}(t) \psi(z)
$$

where $a_{j}(t)$ and $b_{j}(t)$ are functions of time only. Calculation of Eqs. 18 and 19 yields

$$
\begin{aligned}
& \frac{d^{2} a_{j}}{d t^{2}}+2 \zeta_{v} \omega_{v} \frac{d a_{j}}{d t}+\omega_{v}^{2} a_{j}+\frac{\rho \pi R^{2}}{m} \sum_{k=1}^{n}\left(\alpha_{j k} \frac{d^{2} a_{k}}{d t^{2}}+\sigma_{j k} \frac{d^{2} b_{k}}{d t^{2}}\right) \\
& -\frac{\rho U^{2}}{m \omega} \sum_{k=1}^{n}\left(\alpha_{j k}^{\prime} \frac{d a_{k}}{d t}+\sigma_{j k}^{\prime} \frac{d b_{k}}{d t}\right)-\frac{\rho U^{2}}{m} \sum_{k=1}^{n}\left(\alpha_{j k}^{\prime \prime} a_{k}+\sigma_{j k}^{\prime \prime} b_{k}\right)=0
\end{aligned}
$$

and

$$
\begin{aligned}
& \frac{d^{2} b_{j}}{d t^{2}}+2 \zeta_{v} \omega_{v} \frac{d b_{j}}{d t}+\omega_{v}^{2} b_{j}+\frac{\rho \pi R^{2}}{m} \sum_{k=1}^{n}\left(\tau_{j k} \frac{d^{2} a_{k}}{d t^{2}}+\sigma_{j k} \frac{d^{2} b_{k}}{d t^{2}}\right) \\
& -\frac{\rho U^{2}}{m \omega} \sum_{k=1}^{n}\left(\tau_{j k}^{\prime} \frac{d a_{k}}{d t}+\beta_{j k}^{\prime} \frac{d b_{k}}{d t}\right)-\frac{\rho U^{2}}{m} \sum_{k=1}^{n}\left(\tau_{j k}^{\prime \prime} a_{k}+\beta_{j k}^{\prime \prime} b_{k}\right)=0 .
\end{aligned}
$$

When the dimensionless parameters are 


$$
\begin{aligned}
& U_{v}=\frac{U}{f_{v} D}, \\
& \gamma=\frac{\rho \pi R^{2}}{m} .
\end{aligned}
$$

Equations 22 and 23 become

$$
\begin{gathered}
\ddot{a}_{j}+\gamma \sum_{k=1}^{n}\left(\alpha_{j k} \ddot{a}_{k}+\sigma_{j k} \ddot{b}_{k}\right)+2 \zeta_{v} \omega_{v} \dot{a}_{j}-\frac{\gamma}{\pi^{3}} U_{v}^{2}\left(\frac{\omega_{v}^{2}}{\omega}\right) \sum_{k=1}^{n}\left(\alpha_{j k}^{\prime} \dot{a}_{k}+\sigma_{j k}^{\prime} \dot{b}_{k}\right) \\
\quad+\omega_{v} a_{j}-\frac{\gamma}{\pi^{3}} U_{v}^{2} \omega_{v}^{2} \sum_{k=1}^{n}\left(\alpha_{j k}^{\prime \prime} a_{k}+\sigma_{j k}^{\prime \prime} b_{k}\right)=0
\end{gathered}
$$

and

$$
\begin{aligned}
& \ddot{b}_{j}+\gamma \sum_{k=1}^{n}\left(\tau_{j k} \ddot{a}_{k}+\beta_{j k} \dot{b}_{k}\right)+2 \zeta_{v} \omega_{v} \dot{b}_{j}-\frac{\gamma}{\pi^{3}} U_{v}^{2}\left(\frac{\omega_{v}^{2}}{\omega}\right) \sum_{k=1}^{n}\left(\tau_{j k}^{\prime} \dot{a}_{k}+\beta_{j k}^{\prime} \dot{b}_{k}\right) \\
& +\omega_{v}^{2} b_{j}-\frac{\gamma}{\pi^{3}} U_{v}^{2} \omega_{v}^{2} \sum_{k=1}^{n}\left(\tau_{j k}^{\prime \prime} a_{k}+\beta_{j k}^{\prime \prime} b_{k}\right)=0,
\end{aligned}
$$

where the dot denotes differentiation with respect to $t$.

\subsection{Dynamic Instability}

Once the fluid-force coefficients are known, it is straightforward to analyze the stability of a tube array in crossflow. Equations 26 and 27 can be written

$$
[\mathrm{M}]\{\ddot{\mathrm{Q}}\}+[\mathrm{C}]\{\dot{\mathrm{Q}},\}+[\mathrm{K}]\{\mathrm{Q}\}=\{0\},
$$

or

$$
\left[\mathrm{M}_{\mathrm{s}}+\mathrm{M}_{\mathrm{f}}\right]\{\ddot{\mathrm{Q}}\}+\left[\mathrm{C}_{\mathrm{s}}+\mathrm{C}_{\mathrm{f}}\right]\{\dot{\mathrm{Q}},\}+\left[\mathrm{K}_{\mathrm{S}}+\mathrm{K}_{\mathrm{f}}\{\mathrm{Q}\}=\{0\}\right.
$$

where $\{Q\}$ represents the displacement vectors consisting of $a_{j}$ and $b_{j} ;[M]$ is the mass matrix, including structural mass $\left[\mathrm{M}_{\mathrm{s}}\right]$ and added mass $\left[\mathrm{M}_{\mathrm{f}}\right] ;[\mathrm{C}]$ is the 
damping matrix, including structural damping $\left[\mathrm{C}_{\mathrm{s}}\right]$ and fluid damping $\left[\mathrm{C}_{\mathrm{f}}\right]$; and $[\mathrm{K}]$ is the stiffness matrix, including structural stiffness $\left[\mathrm{K}_{\mathrm{s}}\right]$ and fluid stiffness $\left[\mathrm{K}_{\mathrm{f}}\right]$.

By premultiplying by $\{Q\}^{\mathrm{T}}$ and forming the symmetric and antisymmetric components of the matrices,

$$
\begin{array}{ll}
{\left[\mathrm{M}_{1}\right]=\frac{1}{2}\left([\mathrm{M}]+[\mathrm{M}]^{\mathrm{T}}\right),} & {\left[\mathrm{M}_{2}\right]=\frac{1}{2}\left([\mathrm{M}]-[\mathrm{M}]^{\mathrm{T}}\right),} \\
{\left[\mathrm{C}_{1}\right]=\frac{1}{2}\left([\mathrm{C}]+[\mathrm{C}]^{\mathrm{T}}\right),} & {\left[\mathrm{C}_{2}\right]=\frac{1}{2}\left([\mathrm{C}]-[\mathrm{C}]^{\mathrm{T}}\right),} \\
{\left[\mathrm{K}_{1}\right]=\frac{1}{2}\left([\mathrm{~K}]+[\mathrm{K}]^{\mathrm{T}}\right),} & {\left[\mathrm{K}_{2}\right]=\frac{1}{2}\left([\mathrm{~K}]-[\mathrm{K}]^{\mathrm{T}}\right),}
\end{array}
$$

the terms may be separated, giving

$$
\begin{aligned}
\{\dot{\mathrm{Q}}\}^{\mathrm{T}}\left[\mathrm{M}_{1}\right]\{\ddot{\mathrm{Q}}\} & +\{\dot{\mathrm{Q}}\}^{\mathrm{T}}\left[\mathrm{C}_{2}\right]\{\dot{\mathrm{Q}}\}+\{\dot{\mathrm{Q}}\}^{\mathrm{T}}\left[\mathrm{K}_{1}\right]\{\mathrm{Q}\} \\
& =-\left(\{\dot{\mathrm{Q}}\}^{\mathrm{T}}\left[\mathrm{M}_{2}\right]\{\ddot{\mathrm{Q}}\}+\{\dot{\mathrm{Q}}\}^{\mathrm{T}}\left[\mathrm{C}_{1}\right]\{\dot{\mathrm{Q}}\}+\{\dot{\mathrm{Q}}\}^{\mathrm{T}}\left[\mathrm{K}_{2}\right]\{\mathrm{Q}\}\right) .
\end{aligned}
$$

Equation 31 equates rates of work. Those terms on the right-hand side of the equation produce a net work resultant when integrated over a closed path through space $\{Q\}$, with the magnitude depending on the path taken. The forces corresponding to the matrices $\left[\mathrm{M}_{2}\right],\left[\mathrm{C}_{2}\right]$, and $\left[\mathrm{K}_{2}\right]$, appearing on the right-hand side, are thus, by definition, the nonconservative parts of the forces represented by $[\mathrm{M}]$, $[\mathrm{C}]$, and $[\mathrm{K}]$. The terms on the left-hand side similarly can be shown to give rise to a zero work resultant over any closed path; therefore, together, they are the sum of the rates of work from the potential forces and the rate of change of kinetic energy.

Fluidelastic instability of tube arrays is caused by high-velocity flow; its effects are contained in the matrices $\left[\mathrm{C}_{1}\right],\left[\mathrm{C}_{2}\right],\left[\mathrm{K}_{1}\right]$, and $\left[\mathrm{K}_{2}\right]$. These matrices are functions of fluid-damping and fluid-stiffness coefficients. Various types of dynamic instability can be classified according to the dominant terms in Eq. 31 as follows:

Fluid-damping-controlled instability (single-mode flutter). The dominant terms are associated with the symmetric damping matrix $\left[C_{1}\right]$. The instability arises because the fluid-dynamic forces create negative damping. Parameters $\alpha_{j j}^{\prime}$ and $\beta_{\mathrm{jj}}^{\prime}$ play the most important role in determining the stability-instability boundaries. 
Fluid-stiffness-controlled instability (coupled-mode flutter). The dominant terms are associated with the antisymmetric stiffness matrix $\left[K_{2}\right]$. It is called coupled-mode flutter because a minimum of two modes are required to produce it. In this case, $a_{j k}^{\prime \prime}$ and $\tau_{j k}^{\prime \prime}$ for $j=k$ play the major role in determining the stability characteristics.

From Eqs. 26 and 27 or 28 , the natural frequencies and modal damping ratios of the coupled tube/fluid system can be calculated as follows:

$$
\begin{aligned}
& f_{f}=f_{f}\left(\gamma, \zeta_{v}, U_{v}\right), \\
& \zeta_{f}=\zeta_{f}\left(\gamma, \zeta_{v}, U_{v}\right) .
\end{aligned}
$$

Note that fluid-damping and fluid-stiffness coetficients are functions of $U_{r}\left(=U / f_{f} D\right)$; therefore, a numerical method is needed to calculate $f_{f}$ and $\zeta_{f}$.

The nondimensional parameters in Eqs. 26 and 27 are $\gamma, \zeta_{v}, U_{v}, \alpha_{j k}, \sigma_{j k}, \tau_{j k}, \beta_{j k}$, $\alpha_{j k}^{\prime}, \beta_{j k}^{\prime}, \sigma_{j k}^{\prime}, \tau_{j k}^{\prime}, \alpha_{j k}^{\prime \prime}, \beta_{j k}^{\prime \prime}, \sigma_{j k}^{\prime \prime}$, and $\tau_{j k}^{\prime \prime}$. Therefore, the critical flow velocity can be written in a functional form as

$$
U_{v}=F\left(\gamma, \zeta_{v}, \omega_{v} / \omega, \alpha_{j k}, \sigma_{j k}, \tau_{j k}, \beta_{j k}, \alpha_{j k}^{\prime}, \sigma_{j k}^{\prime}, \tau_{j k}^{\prime}, \beta_{j k}^{\prime}, \alpha_{j k}^{\prime \prime}, \sigma_{j k}^{\prime \prime}, \tau_{j k}^{\prime \prime}, \beta_{j k}^{\prime \prime}\right) .
$$

In the unsteady flow theory, all fluid forces are included and characterized properly and the theory is applicable in all cases regardless of different flow regimes or system parameters. On the other hand, quasistatic and quasisteady flow theories are applicable in some specific parameter ranges, depending on the case. Therefore, the unsteady flow theory can be used to assess the validity of other approximate flow theories of the fluidelastic instability of tube arrays in crossflow.

\subsection{Simplifled Case}

For a given array of tubes, if fluid-force coefficients are independent of $U_{r}$, then

$$
\mathrm{U}_{\mathrm{v}}=\mathrm{F}\left(\gamma, \zeta_{\mathrm{v}}\right)
$$

or

$$
U_{r}=\frac{f_{v}}{f_{f}} F\left(\gamma, \zeta_{v}\right)
$$

i.e., the critical flow velocity is a function of mass ratio and damping ratio only. 
In a light fluid, fluid inertia and damping associated with the quiescent fluid can be neglected. Equations 26 and 27 can be written

$$
\begin{aligned}
& \ddot{a}_{j}+2 \zeta_{v} \omega_{v} \dot{a}_{j}+\omega_{v}^{2} a_{j}-\frac{\gamma}{\pi^{3}} U_{v}^{2} \sum_{k=1}^{n}\left[\frac{\omega_{v}^{2}}{\omega}\left(\alpha_{j k}^{\prime} \dot{a}_{k}+\sigma_{j k}^{\prime} \dot{b}_{k}\right)+\omega_{v}^{2}\left(\alpha_{j k}^{\prime \prime} a_{k}+\sigma_{j k}^{\prime \prime} b_{k}\right)\right]=0, \\
& \ddot{b}_{j}+2 \zeta_{v} \omega_{v} \dot{b}_{j}+\omega_{v}^{2} b_{j}-\frac{\gamma}{\pi^{3}} U_{v}^{2} \sum_{k=1}^{n}\left[\frac{\omega_{v}^{2}}{\omega}\left(\tau_{j k}^{\prime} \dot{a}_{k}+\beta_{j k}^{\prime} \dot{b}_{k}\right)+\omega_{v}^{2}\left(\tau_{j k}^{\prime \prime} a_{k}+\beta_{j k}^{\prime \prime} b_{k}\right)\right]=0
\end{aligned}
$$

In a light fluid, all fluid-force coefficients are approximately independent of $U_{r}$ and the oscillation frequency is $\cong \omega_{v}\left(i . e ., \omega_{v}=\omega_{f}\right) ; \gamma U_{v}^{2}$ plays the same role as $\zeta_{v}$, both of them contributing to system damping. The modal damping for a particular mode can be written

$$
\zeta_{f}=\zeta_{v}-C \gamma U_{v}^{2}
$$

where $\mathrm{C}$ depends on fluid-damping and fluid-stiffness coefficients. Instability occurs if $\zeta_{f}=0$; i.e.,

$$
\mathrm{U}_{\mathrm{v}}=\frac{1}{\mathrm{C}}\left(\frac{\zeta_{v}}{\gamma}\right)^{0.5}
$$

or

$$
\frac{U}{f_{v} D} \propto\left(\frac{2 \pi \zeta_{v} m}{\rho D^{2}}\right)^{0.5}
$$

Thus, the critical flow velocity is a function of the mass-damping parameter and proportional to its half-power.

\section{Closing Remarks}

In the unsteady flow theory, all fluid force components are included and quantified properly and the theory is applicable in all cases for all system parameters. On the other hand, quasistatic and quasisteady flow theories are applicable in some specific parameter ranges because of the approximations made in 
the characterization of flow effect. Therefore, the unsteady flow theory is the only theory that can be used to assess the validity of other approximate flow theories on fluidelastic instability of tube arrays in crossflow.

Tanaka and his colleagues $(1980,1981,1982)$ in Japan were the first group to measure the motion-dependent fluid forces acting on tube arrays, which are the critical elements of fluidelastic instability. In their studies, no effort was made to develop the fluid-force coefficients. It appears that the group at Argonne National Laboratory was the first team to develop the nondimensional motion-dependent fluid-force coefficients for tube arrays in crossflow. It also has been verified that at high reduced flow velocity, these coefficients are independent of reduced flow velocity. Knowing this characteristic significantly reduces the effort necessary to develop prediction models.

In earlier studies, only a portion of the motion-dependent fluid forces was measured. In the applications of fluid forces to predict fluidelastic instability, several assumptions must be made about the symmetry or asymmetry of fluid forces. In this study, all fluid-force components were measured. The symmetry or asymmetry of motion-dependent fluid forces is not necessarily correct, and in the future, measurements should be performed to verify the symmetry or asymmetry of fluid forces. Because of the symmetry or asymmetry of fluid forces, the instability of tube arrays subject to reversed flow may be different from that for uniform flow.

Motion-dependent fluid forces are a function of the amplitude of tube oscillations. The amplitudes of oscillations range up to $17 \%$ of tube diameter $(\approx 50 \%$ of tube gap) in tests performed in Japan. The fluid forces measured were practically independent of oscillation amplitudes in this range. In the study reported here, the oscillation amplitudes were up to $=70 \%$ of the tube gap. The effect of oscillation amplitudes on fluid force coefficients was still insignificant.

To develop a reliable design guideline for fluidelastic instability of tube arrays for application to heat exchangers and steam generators, the unsteady flow theory is the only reliable theory to use. The key elements are the motion-dependent fluid force coefficients. In this study, a reliable method was developed and shown capable of providing all necessary fluid force coefficients. A systematic study can now be performed to measure all fluid force coefficients for various tube arrays, including square and triangular arrays with differing pitch-to-diameter ratios. Once the necessary fluid force coefficients are determined, the urioieady flow theory can be applied to practical components for the evaluation of fluidelastic instability. 


\section{Acknowledgments}

This work was jointly funded by Argonne National Laboratory as an FY-1992 Argonne Laboratory Directed Research and Development (LDRD) Program titled "Unsteady Flow Theory for Fluidelastic Instability of Tube Arrays in Crossflow," LDRD Proposal No. 92-046; by Taiwan Power Company under an agreement with the U.S. Department of Energy, contract agreement 31-109-Eng-38-85847; and by the U.S. Department of Energy Office of Basic Energy Sciences, Office of Engineering and Geosciences, under Contract W-31-109-Eng-38.

The authors are grateful to $\mathrm{Mr}$. Roger Smith for his help in setting up the test facility and instrumentation and conducting the experimental work.

\section{References}

Auger, J. L., and Coutanceau, J., 1978, On the Complex Structure of the Downstream Flow of Cylindrical Tube Rows at Various Spacings, Mech. Res. Comm., Vol. 5, pp. 297-302.

Behr, M., Tezduyar, T. E., and Higuchi, H., 1991, Wake Interference Behind Two Flat Plates Normal to the Flow: A Finite.Element Study, Theoretical Computational Fluid Dyn., Vol. 2, pp. 223-250.

Blevins, R. D., 1977, Fluidelastic Whirling of Tube Rows and Tube Arrays, ASME J. Fluids Eng., Vol. 99, pp. 457-461.

Borges, A. R. J., 1969, Vortex Shedding Frequencies of the Flow Through Two-Row Banks of Tubes, J. Mech. Eng. Sci., Vol. 11, pp. 498-502.

Chen, S. S., 1975, Vibration of Nuclear Fuel Bundles, Nucl. Eng. Des., Vol. 35, pp. 399.422.

Chen, S. S., 1976, Crossflow.Induced Vibrations of Heat Exchanger Tube Banks, Nucl. Eng. Des., Vol. 47, pp. 67-86.

Chen, S. S., 1987a, Flow.Induced Vibration of Circular Cylindrical Structures, Hemisphere Publishing Corp., New York.

Chen, S. S., 1987b, A General Theory for Dynamic Instability of Tube Arrays in Crossflow, J. Fluids Struct., Vol. 1, pp. 35-53. 
Chen, Y. N., 1968, Flow.Induced Vibration and Noise in Tube Bank Heat Exchangers due to von Karman Streets, ASME J. Eng. Ind., Vol. 90, pp. 134-146.

Clasen, P., and Gregorig, R., 1971, Ein Schuingungskriterium eines Guer. angestromten Rohres, Chem. Ing. Tech., Vol, 43, pp. 982-985.

Connors, H. J., 1970, Fluidelastic Vibration of Tube Arrays Excited by Cross Flow, in Flow Induced Vibration in Heat Exchangers, D. D. Reiff, ed., ASME, New York, pp. $42-56$.

Funakawa, H., Tshimatsu, T., Kumon, K., and Ochiai, T., 1990, Vibration of Tube Arrays Induced by Two.Phase Normal Flow, JSME Int. J., Series III, Vol. 33, pp. 483-487.

Granger, S., 1990, A New Signal Processing Method for Investigating Fluidelastic Phenomena, J. Fluids Struct., Vol. 4, pp. 73-97.

Hara, F., 1987, Unsteady Fluid Dynamic Forces Acting on a Single Row of Cylinders Vibrating in a Cross Flow, in Flow.Induced Vibrations-1987, M. K. Au-Yang and S. S. Chen, eds., PVT.Vol. 122, pp. 51-58.

Holzdeppe, D., and Ory, H., 1988, Reconstruction of Instationary Wind Load Distribution on Structures from Measured Structural Response Time Histories, J. Wind Eng. Ind. Aerodyn., Vol. 28, pp. 231-240.

Ishigai, S., Nishikawa, E., and Yagi, E., 1973, Structures of Gas Flow and Vibration in Tube Banks with Tube Axes Normal to Flow, Int. Symp. on Marine Eng., Tokyo, pp. 1-5-23 to 1-5-33.

Jendrzejczyk, J. A., and Chen, S. S., 1987, Motion-Dependent Fluid Forces Acting on a Tube Row in Crossflow, in Mechanical Signature Analysis: Machinery Vibration, Flow-Induced Vibration, and Acoustic Noise Analysis, DE-Vol. 7, ASME, New York, pp. 233-242.

Lever, J. H., and Weaver, D. S., 1982, A Theoretical Model for the Fluidelastic Instability in Heat Exchanger Tube Bundles, ASME J. Pressure Vessel Technol., Vol. 104, pp. 147-158.

Lever, J. H., and Weaver, D. S., 1984, On the Stability Behaviour of Heat Exchanger Tube Bundles: Part 1-Modified Theoretical Model, Part 2.Numerical Results and Comparison with Experiments, in Proceedings ASME Symp. on Flow-Induced Vibrations, Vol. 2, New York, pp. 83-116. 
Marn, J., and Catton, I., 1991, Stability of Finite Cylinder Array Subjected to Single Phase Cross Flow, 1991 Winter Annual Meeting, PVP-224/FED-126, pp. 1.4.

Paidoussis, M. P., Mavriplis, D., and Price, S. J., 1984, A Potential-Flow Theory for the Dynamics of Cylinder Arrays in Cross-Flow, J. Fluid Mech., Vol. 146, pp. 227-252.

Paidoussis, M. P., Price, S. J., and Mavriplis, D., 1984, A Semi.Potential Flow Theory for the Dynamics of Cylinder Arrays in Cross Flow, in Proceedings ASME Symp. on Flow-Induced Vibrations, Vol. 2, New York, pp. 67-81.

Price, S. J., and Paidoussis, M. P., 1983, Fluidelastic Instability of an Infïnite Double Row of Circular Cylinders Subjected to a Uniform Crossflow, ASME J. Vib., Acoustics, Stress Reliability Des., Vol. 105, pp. 59-66.

Price, S. J. and Paidoussis, M. P. 1984. "An Improved Mathematical Model tor the Stability of Cylinder Rows Subject to Cross-Flow," J. Sound Vib., Vol. 97, pp. 615 . 640.

Price, S. J., Paidoussis, M. P., and Sychtera, P. W., 1988, An Experimental Investigation of the Quasi-Steady Assumption for Bluff Bodies in Cross.Flow, in Proceedings of the 1988 Int. Symp. on Flow-Induced Vibration and Noise, Vol. 1, ASME, New York, pp. 91-111.

Tanaka, H., 1980, Study on Fluidelastic Vibration of Tube Bundle, Nihon Kikai Gakkai Ronbunshu, or Japan Soc. Mech. Eng., Section B, Vol. 46, pp. 1398-1407.

Tanaka, H., and Takahara, S., 1981, Fluid Elastic Vibration of Tube Array in Cross Flow, J. Sound Vib., Vol. 77, pp. 19-37.

Tanaka, H., Takahara, S., and Ohta, K., 1982, Flow.Induced Vibration of Tube Arrays with Various Pitch-to-Diameter Ratios, Trans. ASME, J. Pressure Vessel Technol., Vol. 104, pp. 168-174.

Teh, C. E., and Goyder, H. G. D., 1988, Data for the Fluidelastic Instability of Heat Exchanger Tube Bundles, in Flow-Induced Vibration and Noise in Cylinder Arrays, ASME Publication, New York, po. 77-94.

Van Der Hoogt, P. J. M., and Van Campen, D. H., 1984, Self.Induced Instabilities of Parallel Tubes in Potential Cross-Flow, in Proceedings ASME Symp. on Flow. Induced Vibrations, Vol. 2, New York, pp. 53-66. 
Xie, Joming, 1988, CVR Method for Identification of Nonsteady Aerodynamic Model, J. Wind Eng. Ind. Aerodyn., Vol. 29, pp. 389-397.

Yetisir, M., and Weaver, D. S., 1988, On an Unsteady Theory for Fluidelastic Instability of Heat Exchanger Tube Arrays, 1988 Int. Symp. on Flow-Induced Vibrations and Noise, Vol. 3, ASME, New York, pp. 181-195.

Yetisir, M., and Weaver, D. S., 1992, A Theoretical Study of Fluidelastic Instability in a Flexible Array of Tubes, 3rd Int. Symp. on Flow-Induced Vibration and Noise, Vol. 2, Cross-Flow-Induced Vibration of Cylinder Arrays, ASME, New York. 


\section{Distribution for ANL-93/15}

\section{Internal}

Y. Cai

S. S. Chen (20)

H. Drucker

J. A. Jendrzejczyk (5)

C. A. Malefyt

R. B. Poeppel
D. M. Rote

R. A. Valentin

M. W. Wambsganss (3)

R. W. Weeks

S. Zhu (5)

TIS Files

\section{External}

DOE-OSTI for distribution per UC-406 (45)

ANL Libraries
ANL-E
ANL-W

Manager, Chicago Field Office, DOE

Director, Technology Management Div., DOE-CH

D. L. Bray, DOE-CH

A. L. Taboas, DOE-CH

Energy Technology Division Review Committee:

H. K. Birnbaum, University of Illinois at Urbana-Champaign, Urbana

R. C. Buchanan, University of Cincinnati, Cincinnati, OH

M. S. Dresselhaus, Massachusetts Institute of Technology, Cambridge, MA

B. G. Jones, University of Illinois at Urbana-Champaign, Urbana

C.-Y. Li, Cornell University, Ithaca, NY

S.-N. Liu, Fremont, CA

R. E. Smith, Engineering Applied Sciences, Inc., Trafford, PA

W. H. Chang, Taiwan Power Company, Taiwan

P. C. Chen, Power Research Institute, Taiwan

J. S. Coleman, DOE, Washington, DC

S. K. Datta, DOE, Washington, DC

Ching-Nan Fan, Institute of Nuclear Energy Research, Taiwan

Y. C. Liao, Taiwan Power Company, Taiwan

Ming-Hui Lin, Taiwan Power Company, Taiwan

O. P. Manley, DOE, Washington, DC

1. C. Moon, Conmell University, Ithaca, NY 

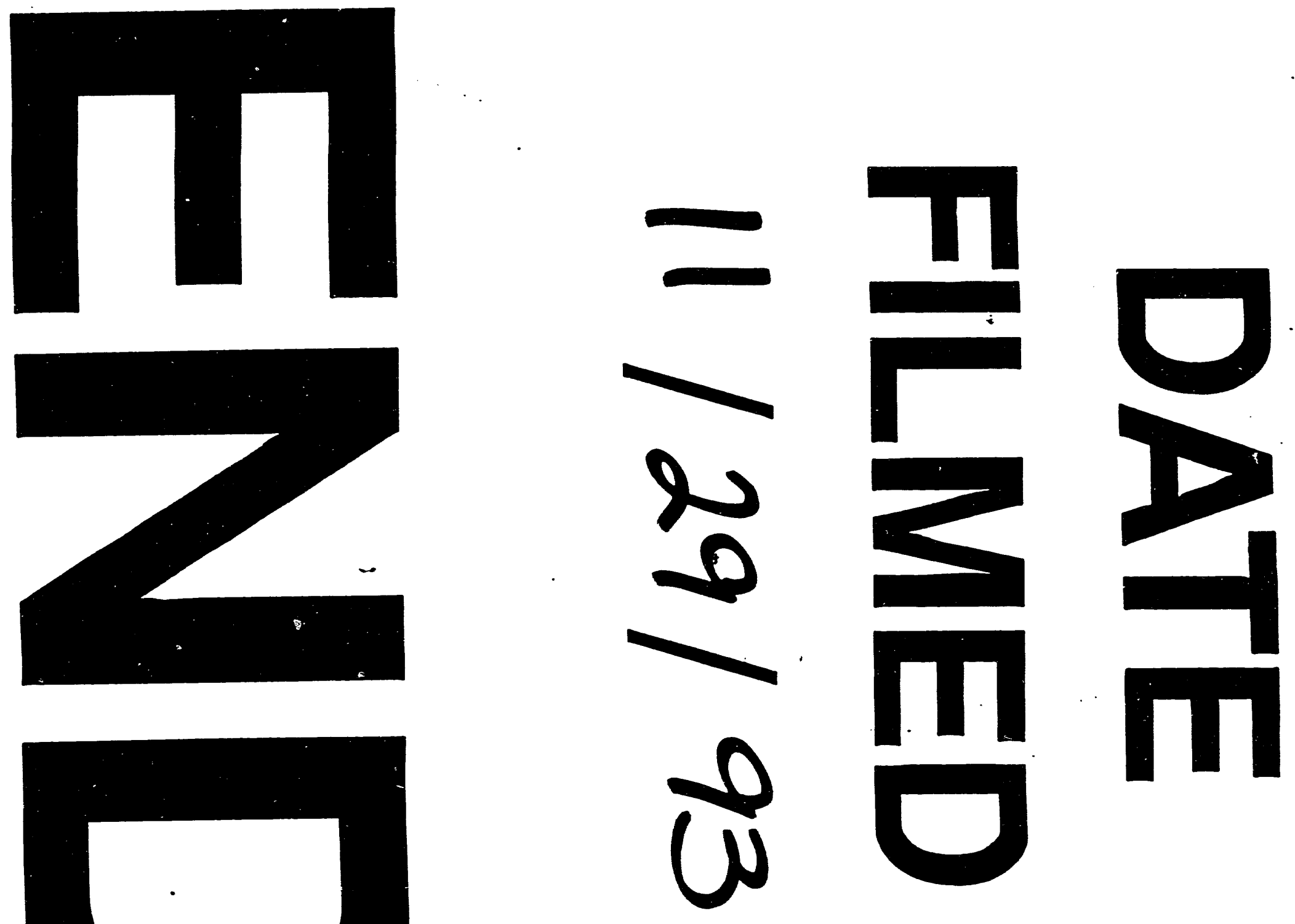
